REVIEW ARTICLE

\title{
Internal transport barriers in tokamak plasmas*
}

\author{
R C Wolf ${ }^{1}$ \\ Max-Planck-Institut für Plasmaphysik, EURATOM Association, D-85748 Garching, Germany \\ E-mail: r.wolf@fz-juelich.de
}

Received 2 April 2002

Published 29 November 2002

Online at stacks.iop.org/PPCF/45/R1

\begin{abstract}
Internal transport barriers in tokamak plasmas are explored in order to improve confinement and stability beyond the reference scenario, used for the ITER extrapolation, and to achieve higher bootstrap current fractions as an essential part of non-inductive current drive. Internal transport barriers are produced by modifications of the current profile using external heating and current drive effects, often combined with partial freezing of the initial skin current profile. Thus, formerly inaccessible ion temperatures and $Q_{\mathrm{DT}}^{\mathrm{eq}}$ values have been (transiently) achieved. The present paper reviews the state of the art of these techniques and their effects on plasma transport in view of optimizing the confinement properties. Implications and limits for possible steady state operations and extrapolation to burning plasmas are discussed.
\end{abstract}

\section{Contents}

1. Introduction 2

1.1. Ignition condition $\quad 3$

1.2. Fusion power density 6

1.3. Bootstrap current

2. Anomalous transport and characterization of internal transport barriers 9

2.1. Anomalous transport and profile stiffness 9

2.2. Temperature gradient modes and their stabilization $\quad 13$

2.3. Characterization of internal transport barriers 20

3. Survey of plasma regimes with internal transport barriers 30

3.1. Internal transport barriers with $T_{\mathrm{i}}>T_{\mathrm{e}} \quad 32$

3.2. Internal transport barriers with $T_{\mathrm{i}}<T_{\mathrm{e}} \quad 45$

3.3. Internal transport barriers with $T_{\mathrm{i}} \approx T_{\mathrm{e}} \quad 49$

* This report was submitted for the degree d'Aǵregé de l'Enseignement Supérieur at the University of Mons-Hainaut, Belgium.

1 Present address: Institut für Plasmaphysik, Forschungszentrum Jülich GmbH, Assoc. EURATOM/FZJ, Trilateral Euregio Cluster, D-52425 Jülich, Germany. 
4. Internal transport barrier dynamics $\quad 55$

4.1. Internal transport barrier formation and sustainment $\quad 55$

4.2. Impurity transport $\quad 66$

$\begin{array}{ll}\text { 4.3. MHD stability } & 68\end{array}$

5. Summary and conclusions $\quad 74$

$\begin{array}{ll}\text { 5.1. ITB definitions } & 75\end{array}$

$\begin{array}{ll}\text { 5.2. Experiments } & 75\end{array}$

$\begin{array}{ll}\text { 5.3. Steady state } & 77\end{array}$

Acknowledgments $\quad 80$

$\begin{array}{ll}\text { Appendix } & 81\end{array}$

Appendix A.1. Tokamak experiments $\quad 81$

Appendix A.2. Estimate for $Q_{\mathrm{DT}}(R) \quad 81$

Appendix A.3. Radial electric field $\quad 83$

$\begin{array}{ll}\text { References } & 85\end{array}$

\section{Introduction}

The main goal of magnetic confinement fusion research is to develop an electricity producing power plant based on the thermonuclear fusion reaction between deuterium and tritium nuclei. The reaction products are helium nuclei, also called $\alpha$-particles, which are supposed to transfer their energy to the fusion plasma, and neutrons, which are not confined by the magnetic fields and have to be used to breed the tritium from lithium. The two main confinement concepts which have proved successful are the stellarator (Wagner et al 1995, Carreras et al 1988, Grieger et al 1992, Boozer 1998) and the tokamak (Wesson 1997). Both generate a magnetohydrodynamic (MHD) equilibrium by superimposing poloidal and toroidal magnetic fields in a toroidal configuration to confine the hot plasma along the magnetic field lines. The first uses magnetic fields produced predominantly by external field coils, while the latter is based on a combination of a toroidal magnetic field, also generated by external coils, and a poloidal magnetic field, which requires a toroidal plasma current. This current is generally induced by the flux change in the centrally arranged transformer coils. The charged particles in the plasma, namely the fuel ions, a certain fraction of impurity ions, and electrons, are confined by the magnetic field lines, as they can move close to freely only parallel to the magnetic field. In the broadest sense, the perpendicular cross-field, or radial transport is produced by collisional processes and MHD instabilities. Micro-instabilities which are the small time and spatial scale counterpart to macroscopic MHD instabilities, are thought to be responsible for the turbulent transport, which usually lies above the intrinsic collision induced level.

Since the late 1960s, when the principal capability of a tokamak to magnetically confine a plasma was confirmed by the measurement of $1 \mathrm{keV}$ electron temperature in the Kurchatov Institute T-3 tokamak ${ }^{2}$ (Peacock et al 1969), the development of the tokamak as the leading confinement concept has made continuous progress (Wesson 1997). After some disappointing experience with reduced confinement under strong plasma heating, in 1982 the high confinement or H-mode was discovered (Wagner et al 1982) which improved the prospects of reducing the size of a tokamak required for ignition. In 1992 tritium was used for the first time in a magnetic confinement device (JET Team 1992), first at concentrations much below the $50 \%$ required in a fusion reactor, followed by experiments with equal amounts of tritium and deuterium in 1994 (Strachan et al 1994), culminating in the to date highest fusion power

2 The parameters of most tokamak experiments mentioned in this article are listed in table A1 in the appendix (appendix A.1). 
of $16 \mathrm{MW}$ achieved in JET in 1997 (Keilhacker et al 1999). The $16 \mathrm{MW}$ of fusion power were generated by a heating power of $26 \mathrm{MW}$, corresponding to a power multiplication factor of $Q_{\mathrm{DT}}=0.6$, which is the ratio of fusion power to heating power supplied to the plasma ${ }^{3}$. Under quasi-stationary conditions the fusion power decreased to $4 \mathrm{MW}$ which is nevertheless equivalent to $Q_{\mathrm{DT}} \approx 0.2$ (Gibson et al 1998). In pure deuterium plasmas the equivalent power multiplication factor $Q_{\mathrm{DT}}^{\mathrm{eq}}$ is based on the extrapolation to a deuterium-tritium mixture, neglecting possible effects of the different atomic mass of tritium on confinement (Yushmanov et al 1990). In transient confinement regimes, taking into account the rise of plasma energy as a power loss term, breakeven, i.e. $Q_{\mathrm{DT}}^{\mathrm{eq}}=1$, has already been reached or even slightly exceeded in JET (JET Team 1992) and JT-60U (Ishida et al 1997, Fujita et al 1999).

\subsection{Ignition condition}

The ignition condition is derived from the requirement that the power loss of the plasma must be compensated by the externally supplied heating power and the $\alpha$-particle power from the fusion reaction (Wesson 1997). It can be written in the form of the so-called triple product

$$
n T \tau_{\mathrm{E}}>5 \times 10^{21} \mathrm{~m}^{-3} \mathrm{keV} \mathrm{s} .
$$

The threshold value given in relation (1) is for parabolic density and temperature profiles, where $n$ and $T$ are the corresponding peak values of deuteron density and temperature. In addition, an ion temperature of $T \approx 10 \mathrm{keV}$ is assumed, at which the fusion power at constant plasma pressure exhibits a maximum (for deuterium-tritium fuel) and hence the ignition condition a minimum with regard to the ion temperature. Any fuel dilution by impurity ions would increase the necessary $n T \tau_{\mathrm{E}}$. With respect to the triple product, the highest performance plasmas of present day tokamaks are about one order of magnitude below ignition (ITER Physics Expert Groups 1999). In these cases, however, the ion temperature exceeds that of the electrons considerably, which is not directly transferable to a burning plasma, as the $\alpha$-particles due to their high energy will predominantly heat the electrons, and the electron-ion equilibration time $(\approx 0.5 \mathrm{~s})$ will be much shorter than the expected confinement time $(\approx 6 \mathrm{~s}$, (Kardaun 1999,ITER Physics Expert Groups 1999)).

Due to the lack of a comprehensive transport theory, empirical scaling laws are used to extrapolate from present day tokamaks to a future device. The amount of fusion power to heating power, $Q_{\mathrm{DT}}$, can be estimated from these scaling laws, which relate the energy confinement time, $\tau_{\mathrm{E}}$, of present day tokamaks to plasma and engineering parameters, such as plasma current, $I$, heating power, $P$, or major radius, $R$, of the device (Goldston 1984, Yushmanov et al 1990, H-mode Database Working Group 1994, ITER Confinement Database and Modelling Working Group 1997). A generic example is

$$
\tau_{\mathrm{E}} \sim \frac{I R^{2}}{\sqrt{P}} .
$$

Assuming constant aspect ratio, plasma elongation and safety factor at the plasma boundary, $Q_{\text {DT }}$ essentially depends on the toroidal magnetic field, the quality of the plasma confinement ${ }^{4}$ and the size of the tokamak. Based on a simplified analytical estimate, which is derived in the appendix (section A.2), and assuming that the design parameters of the burning

\footnotetext{
3 The quoted value of 0.6 does not include the rate of change of the plasma energy, which, at the transient plasma conditions at which this fusion power was reached, increases $Q_{D T}$.

4 The confinement quality is usually expressed by the confinement multiplier or $\mathrm{H}$-factor, describing the factor by which $\tau_{\mathrm{E}}$ of a given scaling is exceeded. If one refers, for instance, to the ITER89-P L-mode confinement scaling (Yushmanov et al 1990), then $H_{\mathrm{L}}=1$ for L- and $H_{\mathrm{L}}=2$ for normal H-mode confinement.
} 
(a)

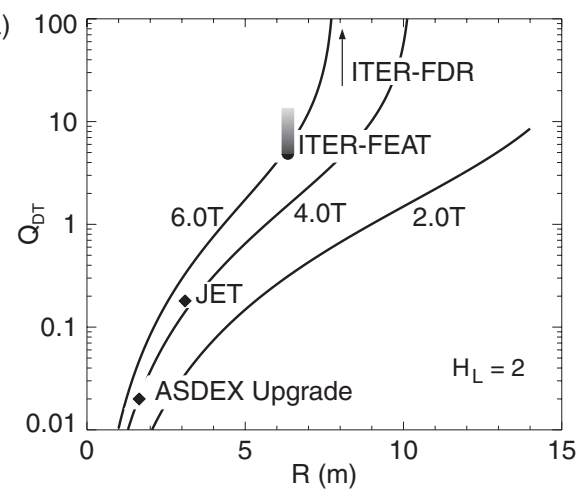

(b)

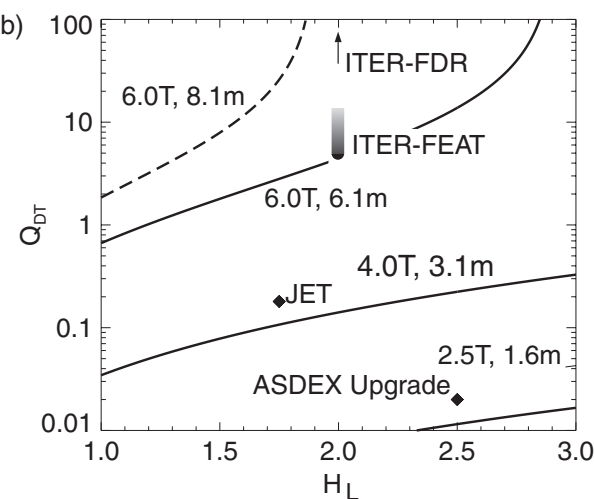

Figure 1. Approximate dependence of the fusion power multiplication factor $Q_{\mathrm{DT}}$ on $(a)$ the major radius $R$ of the tokamak, and $(b)$ the $\mathrm{H}$-factor (referring to L-mode confinement). The various curves, representing the different toroidal magnetic fields and major radii of the tokamak devices considered, are based on an analytical expression for $Q_{\mathrm{DT}}(R, H)$, assuming constant aspect ratio, plasma elongation and safety factor at the plasma boundary (see section A.2). Also assuming that the ITER-FDR parameters (ITER Physics Expert Groups 1999) would lead to ignition $\left(Q_{\mathrm{DT}} \rightarrow \infty\right)$, the thus derived $Q_{\mathrm{DT}}$ values of the other tokamaks are well described by this analytical estimate. The shown operating points include the calculated $Q_{\text {DT }}$ range of ITER-FEAT (Campbell 2001) (shaded area), a steady state JET deuterium-tritium plasma (Gibson et al 1998) and an ASDEX Upgrade steady state high performance plasma. The latter is an extrapolation from a pure deuterium plasma to a mixture of deuterium and tritium.

plasma experiment ITER-FDR ${ }^{5}$ (ITER Physics Expert Groups 1999) would lead to ignition $\left(Q_{\mathrm{DT}} \rightarrow \infty\right)$, this is shown in figure 1 .

The various calculated curves in figure 1 represent $Q_{\mathrm{DT}}$ for the different toroidal magnetic fields and major radii of the tokamak devices considered. They include the two design studies ITER-FDR and ITER-FEAT and the already existing tokamak experiments JET and ASDEX Upgrade. Despite the underlying approximations, the ITER-FEAT scenarios ${ }^{6}$ (Campbell 2001) are well reproduced. The shaded bar indicates the range of possible $Q_{\mathrm{DT}}$ values, considering different fractions of non-inductive current drive. The JET point corresponds to a steady state $\mathrm{H}$-mode discharge, actually using the relevant mixture of deuterium and tritium (Gibson et al 1998). The ASDEX Upgrade point represents a steady state high performance discharge (Wolf et al 2001a), extrapolating from deuterium to deuterium tritium operation. Both, the experimental values of $Q_{\mathrm{DT}}$ from JET and ASDEX Upgrade reflect the general trend quite well. Besides the simplifications made in the calculation of $Q_{\mathrm{DT}}$, the violation of some of the assumptions made in the calculation, e.g. $T_{\mathrm{i}}=T_{\mathrm{e}}=10-20 \mathrm{keV}$, may also add to the deviations.

Both, the toroidal magnetic field and the $\mathrm{H}$-factor, enter with the same weight. Hence, raising $B_{\mathrm{T}}$ or $H$ by the same fraction causes the same increase in $Q_{\mathrm{DT}}$. In a tokamak with superconducting coils, however, the natural limit of $B_{\mathrm{T}}$ is given by the critical field above which superconductivity cannot be maintained. Considering the $1 / R$ fall-off of $B_{\mathrm{T}}$ in a tokamak, a critical field of about $10-12 \mathrm{~T}$ at the high field side of the toroidal magnetic field coils results in a maximum field strength of approximately $6 \mathrm{~T}$ in the plasma centre (ITER Physics Expert

5 ITER-FDR stands for International Thermonuclear Experimental Reactor Final Design Report. This device with a major radius of $R=8.1 \mathrm{~m}$ and a toroidal magnetic field at $R$ of $6 \mathrm{~T}$ was designed to ignite.

6 Compared to ITER-FDR, the size of the device has been reduced in the ITER-FEAT design, decreasing the extrapolated values of $Q_{\mathrm{DT}}$, but still retaining a fusion power significantly above the auxiliary heating power $\left(Q_{\mathrm{DT}}=5-10\right.$ in H-mode). 
Groups 1999). Any additional increase of $Q_{\mathrm{DT}}$ at a given major radius of the device can thus only be achieved by improving the plasma confinement, that is, the H-factor. In other words, a more compact fusion reactor, based on the tokamak design, would require an improvement of the energy confinement.

The toroidal magnetic field and the plasma current are coupled by the safety factor $q$ which, as its name already indicates, is an important measure for the stability of the magnetic field configuration (Freidberg 1982). In divertor tokamaks the safety factor close to the plasma boundary is usually expressed in terms of

$$
q_{95}=g\left(\delta, \kappa, \frac{R}{a}\right) \frac{a^{2} B_{\mathrm{T}}}{R I_{\mathrm{p}}},
$$

which is the value at $95 \%$ of the magnetic flux, since, at the separatrix, $q$ approaches infinity due to the poloidal magnetic field null. $B_{\mathrm{T}}, I_{\mathrm{p}}, R$ and $a$ are the toroidal magnetic field, the plasma current, major and minor radius of the plasma, respectively. $g$ is a function which depends on the aspect ratio $(R / a)$, plasma elongation $(\kappa)$ and triangularity $(\delta)$ (ITER Physics Expert Groups 1999). Keeping $q_{95}$ constant, a reduction of the magnetic field $B_{\mathrm{T}}$ is tantamount to a decrease of the plasma current. From figure 1 it follows again that improved energy confinement, i.e. an increase of the $\mathrm{H}$-factor, permits operation at lower plasma current while sustaining the same fusion performance.

For a given $\mathrm{H}$-factor, the reduction of $Q_{\mathrm{DT}}$ due to a decrease of the plasma current is a direct consequence of the approximately linear dependence of $\tau_{\mathrm{E}}$ on $I_{\mathrm{p}}$. However, a lower plasma current has advantages. Since the flux in the Ohmic transformer of a tokamak is limited, the attainable discharge length increases, lowering the plasma current. In addition, if the fraction of the plasma current produced by the flux change in the transformer (Ohmic current) is to be replaced by a combination of external current drive and the intrinsic bootstrap current (Galeev and Sagdeev 1968, Bickerton et al 1971, Peeters 2000), a lower total current is favourable.

So far, in the discussion above, high energy confinement has been motivated in a very general fashion by the desire to limit the size of a tokamak, and extend the pulse length at a fixed flux swing. While a tokamak plasma cannot be brought to ignition by heating through the plasma current alone, as the parallel electrical resistivity of the plasma decreases with increasing temperature, this very feature leads to the prolonging of the duration of the discharge for a given transformer flux swing.

Any auxiliary heating required for reaching the $10 \mathrm{keV}$ plasma temperature, however, leads at first to a deterioration of the confinement (Goldston 1984, Yushmanov et al 1990), which is, therefore, called low confinement or L-mode. The L-mode is governed by a high level of turbulence which enhances the radial transport perpendicular to the magnetic field lines (see section 2). The combination of sufficiently high neutral beam heating power and a divertor configuration led to the discovery of a high confinement mode in the ASDEX tokamak (Wagner et al 1982). This H-mode is characterized by an increase of the pressure gradient at the plasma edge which is associated with a local reduction of the turbulent transport due to shear in the $E \times B$ flow leading to a decorrelation of the underlying fluctuations (Burrell et al 1992 and references therein). The radial pressure profiles of L- and $\mathrm{H}$-mode are sketched in figures 2(a) and $(b)$. While in L-mode the gradients are limited over the whole plasma cross section, the $\mathrm{H}$-mode exhibits a region with large gradients at the edge, therefore, also termed edge transport barrier, but a similarly flat region in the plasma core. It is evident from the pressure profile shown that in H-mode the product of pedestal pressure (see figure 2) and plasma volume already represents large fractions of the stored energy. Following the considerations regarding such an edge transport barrier, an internal transport barrier (ITB) may be regarded as a region with a steep pressure gradient inside the plasma, as illustrated in figure 2(c). A more detailed 


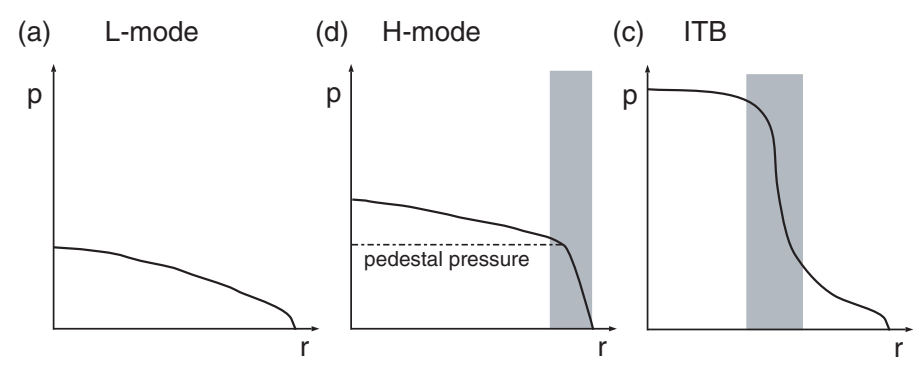

Figure 2. Illustration of pressure profiles observed in (a) L-mode, $(b)$ H-mode and $(c)$ with an internal transport barrier (ITB). The shaded areas indicate regions of reduced radial transport, which in $\mathrm{H}$-mode is located at the plasma edge and for an ITB in the plasma core.

discussion of a possible definition of an ITB also in comparison to L- and H-mode is presented in section 2. In order to attain a similar gain of the internal energy compared to the H-mode, owing to the smaller volume embraced by the internal transport barrier the pressure increase must, accordingly, be larger. If the H-mode edge barrier is combined with an internal transport barrier, the contributions of course add.

\subsection{Fusion power density}

Achieving ignition requires sufficient energy confinement time, $\tau_{\mathrm{E}}$. An additional optimization criterion is inferred from the requirement of adequate fusion power density. The volume averaged fusion power density is the fusion power $P_{\mathrm{f}}$ divided by the plasma volume $V$ :

$$
\frac{P_{\mathrm{f}}}{V}=\frac{1}{4} \overline{n^{2}\langle\sigma v\rangle} \varepsilon_{\mathrm{f}}
$$

where $\langle\sigma v\rangle$ is the fusion reaction rate, $n / 2=n_{\mathrm{D}}=n_{\mathrm{T}}$ the fuel density assuming equal amounts of deuterium (D) and tritium (T) ions and $\varepsilon_{\mathrm{f}}=17.6 \mathrm{MeV}$ the energy per fusion reaction $\left(\overline{n^{2} \ldots}\right.$ denotes the volume average).

An upper limit of $P_{\mathrm{f}} / V$ is given by pressure driven MHD instabilities. The decisive quantity for stability in magnetic confinement devices is the plasma $\beta$, which is the plasma pressure normalized to the magnetic pressure:

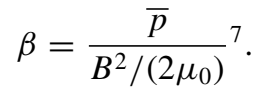

The basic stability limit due to ballooning modes (in the region of first stability) can be expressed in terms of the Troyon scaling (Troyon et al 1984):

$$
\beta_{\max }(\%)=\beta_{\mathrm{N}} \frac{I_{\mathrm{p}}}{a B_{\mathrm{T}}} .
$$

In the region of first stability the normalized $\beta\left(\beta_{\mathrm{N}}\right)$ is restricted to values below approximately three.

7 There are various definitions of $\beta$. The pressure is either a volume or surface average, or the local pressure. If not stated otherwise, here the pressure is equal to the volume average. Regarding the magnetic field normalization, there are three possibilities. The total $\beta$ contains the total magnetic field $B=\sqrt{B_{\phi}^{2}+B_{\theta}^{2}}$, consisting of toroidal $\left(B_{\phi}\right)$ and poloidal $\left(B_{\theta}\right)$ components. $B_{\phi}$ is taken at the magnetic axis and $B_{\theta}$ locally or, if averages are considered, at the plasma boundary. As $B_{\theta} \ll B_{\phi}$ in a tokamak, $\beta \approx \beta_{\phi}$, where in $\beta_{\phi}$ only the toroidal magnetic field is used. Correspondingly, the poloidal $\beta$ is given by $\beta_{\theta}=\bar{p} /\left(2 \mu_{0} B_{\theta}^{2}\right)$. 
Modifications of pressure and $q$ profiles, in particular low or even negative magnetic shear $(s=(r / q)(\mathrm{d} q / \mathrm{d} r))$ allow access to the second stability region, where substantially higher $\beta$ values, at least concerning ballooning stability, can be reached (Strait et al 1995, Rice et al $1996 a, b)$. This feature is one of the advantages of having low or negative magnetic shear, and it is a prerequisite for the formation of the large pressure gradients at the location of internal transport barriers.

Considering the Troyon stability limit, given in equation (6), and $\langle\sigma v\rangle \sim T^{2}$ at $\approx 10 \mathrm{keV}$, where the ignition condition exhibits a minimum (see also relation (1)), the achievable fusion power density becomes

$$
\frac{P_{\mathrm{f}}}{V} \propto\left(\frac{\beta_{\mathrm{N}}}{q_{95}}\right)^{2} .
$$

The explicit dependence of the fusion power density on $B_{\mathrm{T}}$ in this expression has been removed by using the maximum attainable $B_{\mathrm{T}}$ of a superconducting tokamak. According to the confinement criterion for ignition, given above, a normalized measure of confinement can be found

$$
\frac{\beta \tau_{\mathrm{E}}}{a^{2}} \propto\left(\frac{H}{q_{95}}\right)^{2} .
$$

Combining equations (7) and (8) results in the figure of merit $\beta_{\mathrm{N}} H / q_{95}^{2}$ for confinement and fusion power density. Note that the $H^{2} / q^{2}$ dependence is also found in the formula underlying the curves in figure 1, only that there $q_{95}$ is kept constant.

\subsection{Bootstrap current}

Apart from the criteria for the optimization of confinement and fusion power density, another condition regarding the operation of a tokamak in steady state is the requirement for a large fraction of non-inductive current. Although full non-inductive current drive has been achieved in tokamaks by external means (Bernabei et al 1982, Leuterer et al 1985, Anado et al 1986, Saoutic et al 1997, Itoh et al 1999, Sauter et al 2000), due to the limited efficiency of external current drive sources (Pericoli-Ridolfini et al 1999, Oikawa et al 2000 and references therein), in particular at higher electron densities (Gormezano 1993), the maximization of the intrinsic bootstrap current (Galeev and Sagdeev 1968, Bickerton et al 1971, Peeters 2000) is essential. To date current drive efficiencies lie below (Gormezano 1993)

$$
\frac{R n_{\mathrm{e}} I_{\mathrm{CD}}}{P_{\mathrm{CD}}}=0.5 \frac{10^{20} \mathrm{~A}}{W m^{2}},
$$

where $R$ is the major radius of the plasma, $n_{\mathrm{e}}$ the electron density and $P_{\mathrm{CD}}$ the power supplied to the plasma for the purpose of driving the current $I_{\mathrm{CD}}$. Hence, to replace a current of $4 \mathrm{MA}$ in a JET plasma $(R=3.1 \mathrm{~m})$ with a density of $1 \times 10^{20} \mathrm{~m}^{-3}$ entirely by external means would require a power of about $25 \mathrm{MW}$. In a burning fusion plasma with predominantly $\alpha$-particle heating a large current drive power fraction inherently reduces $Q_{\mathrm{DT}}$.

The bootstrap current is associated with the trapped particles in a tokamak plasma and, therefore, is a consequence of the inhomogeneity of the magnetic field strength, which basically falls off like $1 / R$ (Kikuchi and Azumi 1995, Peeters 2000). The underlying mechanism can be thought of as the parallel analogue of the diamagnetic current, which is driven by a density gradient of the trapped particles and subsequently transferred to the passing particles by collisional processes. Depending on the details of heat conductivity and power deposition, a heating and current drive scheme may actually produce more bootstrap current than directly driven current. 
In general, the bootstrap current density is generated by a pressure gradient

$$
j_{\mathrm{BS}} \propto \sqrt{\varepsilon} \frac{\nabla p}{B_{\theta}},
$$

with $\varepsilon$ the inverse aspect ratio and $B_{\theta}$ the poloidal magnetic field component, which is produced by the toroidal plasma current $I_{\mathrm{p}} \propto a B_{\theta}$. Hence, the bootstrap current fraction $f_{\mathrm{BS}}=I_{\mathrm{BS}} / I_{\mathrm{p}}$ becomes

$$
f_{\mathrm{BS}} \propto \sqrt{\varepsilon} \frac{a \nabla p}{B_{\theta}^{2}},
$$

which is proportional to the poloidal $\beta_{\theta}=\bar{p} / B_{\theta}^{2} /\left(2 \mu_{0}\right)$. More general considerations, which include finite aspect ratio (Hirshman 1988) and different collisionality regimes (Hinton and Hazeltine 1976), do not give such a simple dependence on the pressure gradient, but weigh density and temperature gradients differently. Numerical calculations indicate a stronger dependence on the density than the temperature gradient (Houlberg et al 1997, Sauter and Angioni 1999). However, in particular, in the presence of internal transport barriers, the temperature gradient can exceed the density gradient so that nonetheless the temperature gradient contribution can become larger.

In figure 3 the bootstrap current distributions of two different types of pressure profiles are compared (Peeters 2000). The underlying calculations assume constant stored energy and total plasma current. In the conventional regime of operation, which in the plasma core resembles $\mathrm{L}$ - or H-mode confinement, the pressure profile is peaked in the plasma centre, resulting in a broad bootstrap current distribution. The total current density is the sum of bootstrap and Ohmic current contributions, which is provided by the transformer action. In the presence of an ITB the pressure profile develops a strong internal gradient, which produces a bootstrap current peak at the location of the maximum pressure gradient. The stronger gradient of the Ohmic
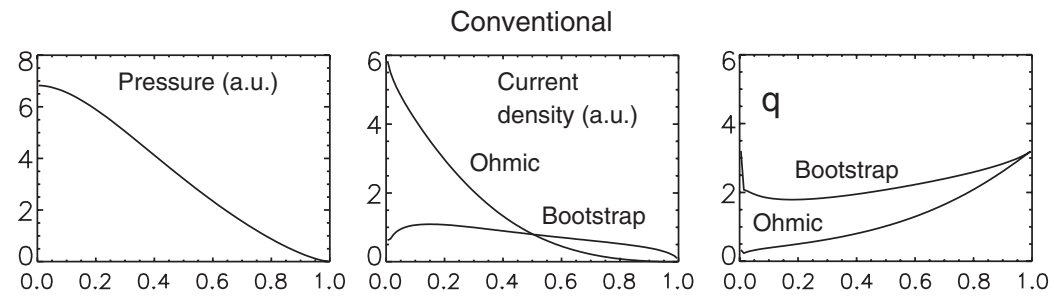

Internal transport barrier
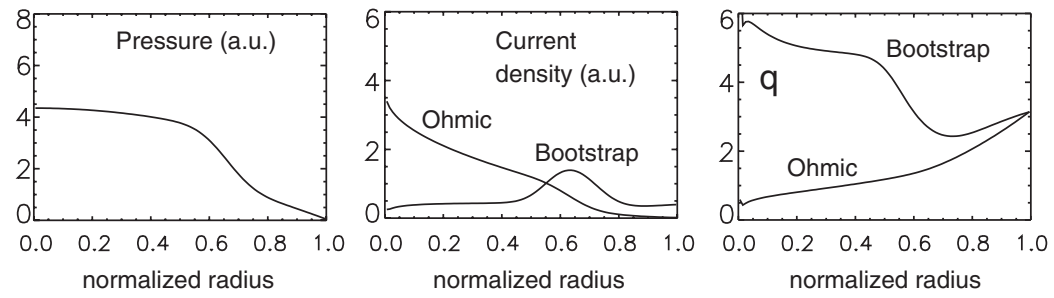

Figure 3. Calculated effect of pressure profile on current distribution (from Peeters (200b)) for a conventional scenario with peaked pressure and current profiles and for an internal transport barrier with a central plateau region and a steep pressure gradient in the plasma core and hence a broad pressure profile. The two cases shown have the same total current $\left(I=\int j \mathrm{~d} A\right)$ and stored energy ( $W=\int p \mathrm{~d} V$ ). The $q$ profiles on the right are calculated, first, assuming an Ohmic current only, and second, including the bootstrap current. The total current is always kept constant, which becomes evident on the unchanged edge $q$. 
current density at the ITB is a consequence of the stronger electron temperature gradient contained in the pressure profile. The plots on the right of figure 3 show the effect of the bootstrap current on the $q$ profile by, first, assuming an Ohmic current only in the calculation, and second, including the bootstrap current. Only in conjunction with the ITB, due to the large off-axis bootstrap current, does the $q$ profile become non-monotonic. As discussed below, the associated negative magnetic shear, $s=(r / q)(\mathrm{d} q / \mathrm{d} r)$, supports the formation of internal transport barriers and hence is consistent with a broad or even hollow current distribution (Kessel et al 1994). Although ideas exist as to how to combine a peaked current distribution also with a large bootstrap current fraction (Taylor 1997), their realization still has to be proven.

The studies to improve confinement and stability in tokamak plasmas are often summarized under the heading advanced tokamak research. The main aims are first, to make a future tokamak reactor more compact, and second, to maximize the non-inductive current drive fraction. These aims are inherently linked. A more compact reactor has to operate at a lower plasma current which requires improved energy confinement to achieve the same $Q_{\mathrm{DT}}$. On the other hand, at given external current drive and bootstrap current a reduction of the plasma current increases the non-inductive current fraction. Ultimately, the maximum bootstrap current is given by the stability limits determining the achievable pressure gradients. Plasma regimes with internal transport barriers can be regarded as a subgroup of the advanced tokamak scenarios (possible definitions of internal transport barriers are discussed in the subsequent sections). Their main advantage is the favourable alignment of pressure and current profiles, required for large bootstrap current fractions. The challenges which lie ahead are the control of the improved confinement state in internal transport barrier regimes to avoid, for instance, detrimental MHD instabilities or undue dilution of the plasma fuel by impurities.

\section{Anomalous transport and characterization of internal transport barriers}

\subsection{Anomalous transport and profile stiffness}

In a magnetically confined plasma the particles are close to moving freely along the magnetic field lines, while perpendicular to them their motion is restricted by the gyration around the magnetic field lines. Binary collisions are the basic mechanisms by which particles and energy are transported across the magnetic field. In a tokamak for typical plasma parameters this collisionally induced, neoclassical transport yields ion thermal conductivities of the order of $\chi_{i} \approx 0.1 \mathrm{~m}^{2} \mathrm{~s}^{-1}$, approximately scaling like $q^{2} Z_{\text {eff }} n_{\mathrm{e}}$, where $q$ is the safety factor, $Z_{\text {eff }}$ the effective ion charge and $n_{\mathrm{e}}$ the electron density (Chang and Hinton 1986). The neoclassical electron heat conductivity is smaller by $\sqrt{m_{\mathrm{e}} / m_{\mathrm{i}}}$ (with $m_{\mathrm{e}} / m_{\mathrm{i}}$ the electron to ion mass ratio) due to the much smaller gyro (or Larmor) radius of the electrons compared to the plasma ions. Mainly driven by ion-electron collisions, the neoclassical particle diffusivity (of the main ion species) is of the order of $\chi_{i}$.

In general, however, neoclassical transport cannot explain the experimentally observed cross-field transport in tokamaks (ITER Physics Expert Groups 1999). In the low confinement regime (L-mode) of auxiliary heated discharges the heat conductivities of both ions and electrons range from 1 to $10 \mathrm{~m}^{2} \mathrm{~s}^{-1}$ and increase from the plasma centre to the edge, exceeding the neoclassical predictions for the ions by one and for the electrons by two orders of magnitude. Also the radial increase of the heat diffusion is not in agreement with neoclassical theory. Although the high confinement regime (H-mode) (Wagner et al 1982), which is associated with the formation of an edge transport barrier, shows a reduction of the transport not only at the plasma edge, but throughout the entire plasma cross section (Burrell et al 1991), the transport remains above neoclassical over large parts of the plasma cross section. 
In the H-mode regime regions where $\chi_{i}$ may approach neoclassical levels are those at the plasma edge and at the very centre. One characteristic of internal transport barriers is that this central region of low ion transport moves or even extends to larger radii (Levinton et al 1995, Rice et al 1996a, Fujita et al 1997a, Söldner et al 1999, Wolf et al 1999a), in the extreme case at least transiently covering most of the plasma volume (Lazarus et al 1996).

This difference between the actual cross-field transport and the neoclassical predictions is attributed to plasma turbulence (Garbet 2001 and references therein) and is called anomalous transport. The turbulence is driven by the free energy sources of a large number of microinstabilities (Connor and Wilson 1994, Yoshizawa et al 2001), essentially initiated by the gradients of temperature and density. Most instabilities which are assumed to be responsible for the plasma turbulence have wave vectors which are oriented mainly perpendicular to the magnetic field lines. Hence, they are supposed to dominate the perpendicular transport, but are of little importance for parallel transport. The validity of parallel neoclassical transport theory is, for instance, confirmed by the good agreement between experimental (Forest et al 1994, Batha et al 1997) and theoretical (Hirshman and Sigmar 1981) parallel electrical resistivities of a tokamak plasma.

Due to the complexity of the physics governing plasma turbulence, a comprehensive theoretical description of anomalous transport does not exist up to now. However, concepts have been devised which are in qualitative and to some extent also in quantitative agreement with experimentally observed phenomena of radial transport.

The main candidates producing anomalous transport are temperature gradient modes, as, on the one hand, they exhibit a threshold behaviour which explains the so-called profile stiffness or profile resilience (Goldston et al 1987, Taylor et al 1989, Baker et al 2001, Hoang et al 2001, Suttrop et al 2001, Ryter et al 2001a) and, on the other hand, their suppression mechanisms are generally consistent with the conditions required for the formation of internal transport barriers. The onset condition for turbulence, driven by temperature gradient modes, is given by a threshold value of the normalized temperature gradient which is proportional to the inverse of the gradient length $L_{\mathrm{T}}$ :

$$
\frac{R}{L_{\mathrm{T}}}=R \frac{|\nabla T|}{T} \text {. }
$$

For convenience, the magnitude signs are omitted in $|\nabla T|$ in the following, always assuming a negative temperature gradient ( $T$ is falling off from the centre to the edge) unless it is stated otherwise. Below the critical value, transport is either neoclassical or dominated by anomalous mechanisms other than temperature gradient modes. Above this threshold the temperature gradient driven turbulence develops, resulting in large levels of radial transport which prevent a further increase of the local temperature gradient.

This means that the resulting heat conductivity is not a property of the plasma, but a consequence of the given heat flux and the temperature at the plasma boundary. This is illustrated in figure 4, for the experimentally determined electron heat transport in JET (Suttrop et al 2001). Above a certain value of $R \nabla T_{\mathrm{e}} / T_{\mathrm{e}}$ an increase of the heat flux results only in a small rise in the logarithmic temperature gradient, but a large rise in the heat conductivity (Baker et al 2001, Ryter et al 2001a), which is indicative of the existence of a critical temperature gradient. The merely small variations of $R \nabla T / T$, when comparing different tokamak experiments (Ryter et al 2001b, Wolf et al 2001b), further supports the conjecture of critical gradient limited profiles. In interpreting the behaviour of the JET data shown, as evidence for profile stiffness, some caution is advised, as also other, less stiff models can describe them (Suttrop et al 2001). The non-linear increase of $Q_{\mathrm{e}}$ with $\nabla T_{\mathrm{e}} / T_{\mathrm{e}}$, however, is beyond question.

In the strict sense the $R / L_{\mathrm{T}}$ criterion is only valid in the limit of flat density profiles. For peaked density profiles it has to be replaced by $\eta=(\nabla|\ln T|) /(\nabla|\ln n|)=L_{T} / L_{n}$, where $n$ 


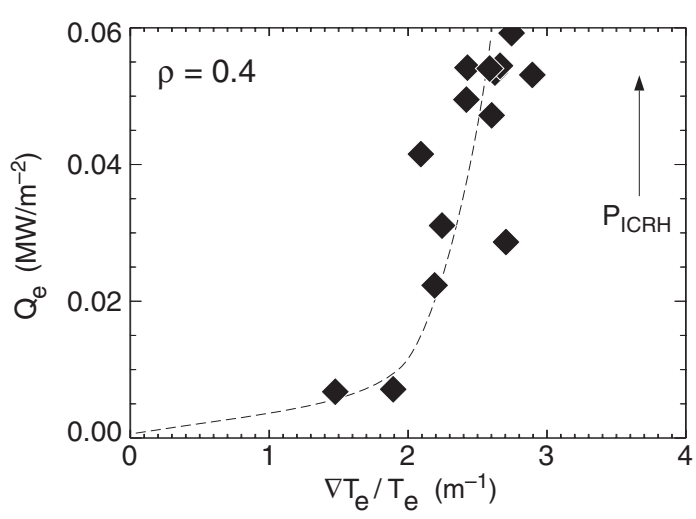

Figure 4. At constant total heating power the electron heat flux, $Q_{\mathrm{e}}$, has been varied in JET by changing the ratio of ion cyclotron resonance heating (ICRH) of the hydrogen minority ions to the neutral beam injection (NBI) power at a constant total heating power of $14 \mathrm{MW}$ (reproduced from (Suttrop et al 2001)). As the minority ions preferably heat the plasma electrons, $Q_{\mathrm{e}}$ increases with rising $P_{\text {ICRH }}$. The strongly non-linear increase of $Q_{\mathrm{e}}$ (at the normalized radius of $\rho=0.4$ ) with $\nabla T_{\mathrm{e}} / T_{\mathrm{e}}$ indicates some kind of critical gradient behaviour.

is the plasma density. Different from the large density gradients at the H-mode edge, in many cases $L_{n}$ is large enough in the plasma core, so that $L_{\mathrm{T}}$ is sufficient to describe the stability condition of the turbulent modes.

The effect of a critical gradient length is exemplified in figure 5 (Ryter et al 2001c) for ASDEX Upgrade high density H-mode discharges. At otherwise similar boundary conditions the heat flux has been varied by changing the acceleration voltage from 60 to $93 \mathrm{kV}$ and hence the power deposition of the neutral beam heating. Nevertheless, the temperature profiles are almost identical (at the high plasma densities $T_{\mathrm{e}}=T_{\mathrm{i}}$ ), resulting in a larger heat conductivity, i.e. a poorer confinement, for the more central deposition at $93 \mathrm{kV}$. This confinement degradation corresponds to a reduction of $H_{\mathrm{L}}$ by about $13 \%$. The complementary case is shown in figure 6 , where the boundary ion temperature has been changed at an approximately constant heat flux. Into both discharges $5 \mathrm{MW}$ of neutral beams are injected at similar line averaged densities, ranging from 4.2 to $4.7 \times 10^{19} \mathrm{~m}^{-3}$. The increase of the edge temperature is due to an H-mode edge barrier, which in the low temperature case has been prevented by limiting the plasma at the inner wall of the torus. In the H-mode the stored energy is about twice as large as that of the L-mode. Because of the same heating power the $\mathrm{H}$-factor is approximately doubled. Maintaining the same $\nabla T / T$ is tantamount to achieving a constant multiplication factor between the two $T_{\mathrm{i}}$ profiles, which is 2.5 here.

Consequently, the boundary condition provided by the edge confinement properties strongly affects the global confinement. Experimental observations support this conjecture that reduced edge transport, which is associated with low edge recycling, correlates with improved core confinement (Strachan et al 1987, Jackson et al 1991, Weisen et al 1991, Ongena et al 1993). In stationary H-mode plasmas edge localized modes (ELMs) (Zohm 1996) fix the edge or pedestal pressure gradient at a certain value (Suttrop et al 1997), depending on the stability margin of the edge instability. If the dependence of the gradient length on the plasma radius does not change, the ratio of any two temperature values at different radial positions is constant. Defining $R / L_{T}=\alpha(\rho)$ this becomes

$$
T(\rho)=T\left(\rho_{\mathrm{b}}\right) \mathrm{e}^{\varepsilon \int_{\rho_{\mathrm{b}}}^{\rho} \alpha\left(\rho^{\prime}\right) \mathrm{d} \rho^{\prime}}=T\left(\rho_{\mathrm{b}}\right) F\left(\rho, \rho_{\mathrm{b}}, \varepsilon\right),
$$



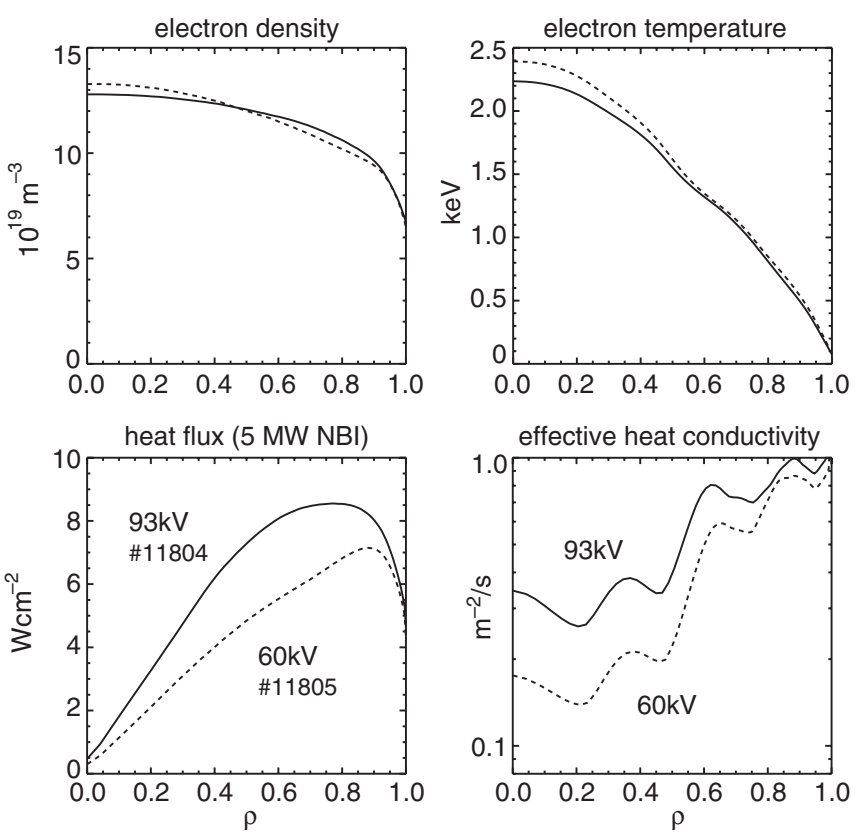

Figure 5. The increase of the heat flux does not result in a change of the local $L_{\mathrm{T}}$ (here of $T_{\mathrm{e}}$ ) in ASDEX Upgrade, but in an increase of the heat conductivity (reproduced from Ryter (2001c); copyright in these materials is vested in the International Atomic Energy Agency, Vienna, Austria from which permission for publication must be obtained). The heat flux has been varied by changing the voltage of the heating beams from 60 (- - -) to $93 \mathrm{kV}$ (- - ). Owing to the slightly lower edge density at $60 \mathrm{kV}$, the corresponding $T_{\mathrm{e}}$ is slightly higher.

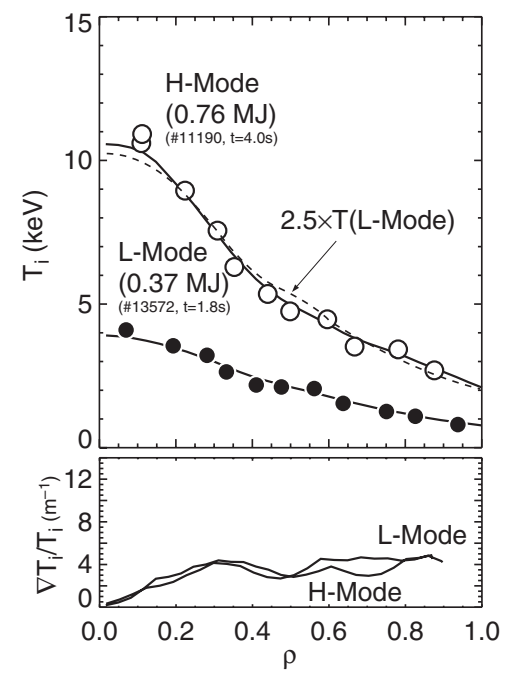

Figure 6. Two ASDEX Upgrade profiles of the ion temperature and its normalized gradient $\nabla T_{\mathrm{i}} / T_{\mathrm{i}}$ for L- and H-mode plasma conditions. Despite a similar heat flux, in the first the H-mode transition is avoided as the plasma is limited at the inner wall of the torus. The constant multiplication factor between the two profiles of 2.5 is clear evidence for the profile resilience or stiffness. The dashed line represents the L-mode profile multiplied by this factor. Only the higher edge temperature in the $\mathrm{H}$-mode case permits the higher core values. 
where $\rho$ is the normalized minor plasma radius, $\rho_{\mathrm{b}}$ its boundary value, for instance at the $\mathrm{H}$-mode pedestal, and $\varepsilon=a / R$ the inverse aspect ratio. Hence, the energy content of the plasma scales with the boundary pressure $p\left(\rho_{\mathrm{b}}\right)=n\left(\rho_{\mathrm{b}}\right) T\left(\rho_{\mathrm{b}}\right)$

$$
W \propto \int_{0}^{\rho_{\mathrm{b}}} n T \rho \mathrm{d} \rho=n\left(\rho_{\mathrm{b}}\right) T\left(\rho_{\mathrm{b}}\right) \int_{0}^{\rho_{\mathrm{b}}} F\left(\rho, \rho_{\mathrm{b}}, \varepsilon\right) \rho \mathrm{d} \rho,
$$

while the integral in this equation depends on the temperature gradient length only, assuming a radially constant plasma density and $T_{\mathrm{e}}=T_{\mathrm{i}}$. This means, that in the presence of stiff temperature profiles the energy of the plasma is solely determined by the pedestal pressure, independent of the ratio of temperature and density at the edge.

Thus, assuming a non-stiff behaviour of the density profile and a temperature gradient length unaffected by the density gradient, any energy increase at constant pedestal pressure has to come from central peaking of the density. This does not consider stabilization of the turbulence by the pressure gradients itself and, therefore, may be valid only within a certain range of pressure gradients.

\subsection{Temperature gradient modes and their stabilization}

Anomalous transport by temperature gradient modes in tokamaks is basically characterized by three different types of microinstabilities, namely the long wavelength $\left(k_{\theta} \rho_{\mathrm{i}} \approx 0.1-0.4\right)$ ion temperature gradient (ITG) mode, the medium wavelength $\left(k_{\theta} \rho_{\mathrm{i}} \approx 0.2-1\right)$ trapped electron mode $\left(\right.$ TEM) and the short wavelength $\left(k_{\theta} \rho_{\mathrm{i}} \geqslant 1\right)$ electron temperature gradient (ETG) mode. Here, $k_{\theta}$ is the poloidal wave number and $\rho_{\mathrm{i}}$ is the ion gyro radius. As elaborated below, the ITG modes are held responsible for the thermal ion, and ETG and trapped electron modes for the thermal electron heat conduction. The interaction between ITG and trapped electron modes produces particle transport, but may also enhance the ion heat conduction.

The stability of these modes depends on various plasma parameters, which include $E \times B$ flows (Burrell 1997 and references therein), magnetic shear, Shafranov shift, electron to ion temperature ratio and impurity content of the plasma (Garbet 2001 and references therein), to name only the most prominent ones. Since these parameters act differently on the different wavelength scales of the turbulent modes, their influence on the respective transport channels also varies.

The turbulent ion heat transport in the core of a tokamak plasma is thought to be dominated by ITG driven modes. The theories of ITG modes (Biglari et al 1989, Romanelli 1989, Garbet et al 1992) predict, even in the limit of flat density profiles, a critical ion temperature gradient $R /\left.L_{T_{\mathrm{i}}}\right|_{\text {crit. }}$ above which the mode is destabilized, leading to the enhanced transport levels ${ }^{8}$. An additional driving mechanism which can reduce the value of $R /\left.L_{T_{\mathrm{i}}}\right|_{\text {crit. }}$ and also enhance the electron transport is provided by TEM, which are driven by the non-adiabatic response of trapped electrons to the perturbed potential of the fluctuations (Redd et al 1999). While pure ITG modes are not directly associated with particle transport, i.e. they do not enhance particle transport and are not excited by density gradients, the trapped electron modes are also driven by density gradients. In fact, contrary to TEM, density gradients can have a stabilizing effect on ITG turbulence. Hence, the overall interaction and mutual excitation or damping of these modes can be rather complex.

Quantitative descriptions of the ion heat conductivities derived from turbulence models have been experimentally verified and in particular recover the observed radial increase of $\chi_{\mathrm{i}}$ (Kotschenreuther et al 1995), which cannot be described by neoclassical theory.

${ }^{8}$ For steep density gradients the relevant quantity describing the marginality of the profiles is $\eta_{i}=\left|\nabla \ln T_{i}\right| /|\nabla \ln n|$. 
The results of a number of transport codes, using various theoretical models of plasma turbulence have been benchmarked in the so-called Cyclone project (Dimits et al 2000), in an attempt to resolve the differences between the theoretical predictions concerning the ignition margin of ITER. Despite the qualitative agreement between the results of various codes, exhibiting a clamping of the logarithmic ion temperature gradient for sufficiently large ion heat fluxes, the quantitative differences for this method of extrapolation are still too large.

The critical gradient at which the temperature gradient modes are destabilized and the saturated mode amplitude at a certain heat flux, which determine the heat conductivity, depend on various plasma parameters, such as magnetic shear, plasma pressure, the ratio of electron to ion temperature and the effective ion charge. A prominent role, at least for the ion transport, is played by sheared $E \times B$ flows, which, by decorrelating the turbulent eddies, can reduce or even suppress the turbulence. Through the force balance equation, the $E \times B$ shear flows themselves depend on various plasma parameters. These dependences are discussed in the following sections.

2.2.1. Current profile and magnetic shear. The details of the current profile play a central role in the transport properties of tokamak plasmas (Kadomtsev and Pogutse 1967). The possible $q$ profiles in a tokamak MHD equilibrium range from monotonic shape to profiles with regions of negative magnetic shear towards the plasma centre (figure 3 ). In conjunction with internal transport barriers the latter are often called negative central shear, negative shear, or reversed shear plasmas. While the core regions of typical L- and H-mode plasmas lie in an intermediate range of positive magnetic shear, both discharges with low or negative and very positive magnetic shear show improved core energy confinement. A rise of the magnetic shear, i.e. an increase of the current profile peaking, is achieved by either ramping down the plasma current (Challis et al 1992, Zarnstorff et al 1993, Kamada et al 1994) or increasing the elongation of the plasma cross section (Lao et al 1993) both fast enough to obtain a deviation of the current profile from its equilibrium distribution, which lasts longer than several energy confinement times. Applying these techniques, the internal inductance $l_{\mathrm{i}}{ }^{9}$ which is a measure for the peaking of the current profile, increases, exhibiting a roughly linear dependence of the $\mathrm{H}$-factor on $l_{\mathrm{i}}$. Internal transport barriers, on the other hand, are observed only with low or negative magnetic shear (Koide et al 1994, Levinton et al 1995, Strait et al 1995, Equipe Tore Supra (presented by X Litaudon) 1996, JET Team (presented by C Gormezano) 1997, de Baar et al 1997, Ishida et al 1997, Buratti et al 1999, Gruber et al 1999a, Pietrzyk et al 2001, Wolf et al 2001a) corresponding to broad or hollow current profiles with low values of $l_{\mathrm{i}}$. This behaviour is reflected in the dependence of the growth rate of the microinstabilities on magnetic shear, as shown in figure 7. For both the high and low positive shear regions the growth rate decreases, corresponding to a reduction of the turbulent transport (Waltz et al 1995, Brunner et al 1998, Wakatani 1998, Maget et al 1999). However, regarding a special role of $s \approx 0$, the various theoretical calculations do not all agree. While some, as the one depicted in figure 7, predict a strong drop in the turbulence at $s \approx 0$ (Wakatani 1998, Maget et al 1999), others merely show a continuing reduction when going from positive to negative magnetic shear (Waltz et al 1995, Brunner et al 1998).

A graphic explanation is given by the magnetic shear dependence of the orientation of convective cells as they map along the magnetic field lines (Antonsen et al 1996). In this picture (figure 8) the transport is largest for intermediate values of shear, where the orientation in major radius direction of the convective cells and hence the extent of the perturbation is best

9 The internal inductance is defined as $l_{\mathrm{i}}=\left(\int \mathrm{d} V B_{\theta}^{2}\right) /\left(V{\overline{B_{\theta}(a)}}^{2}\right)$ where, here, $B_{\theta}(a)$ should be understood as the average poloidal magnetic field on the last closed fluk surface. 


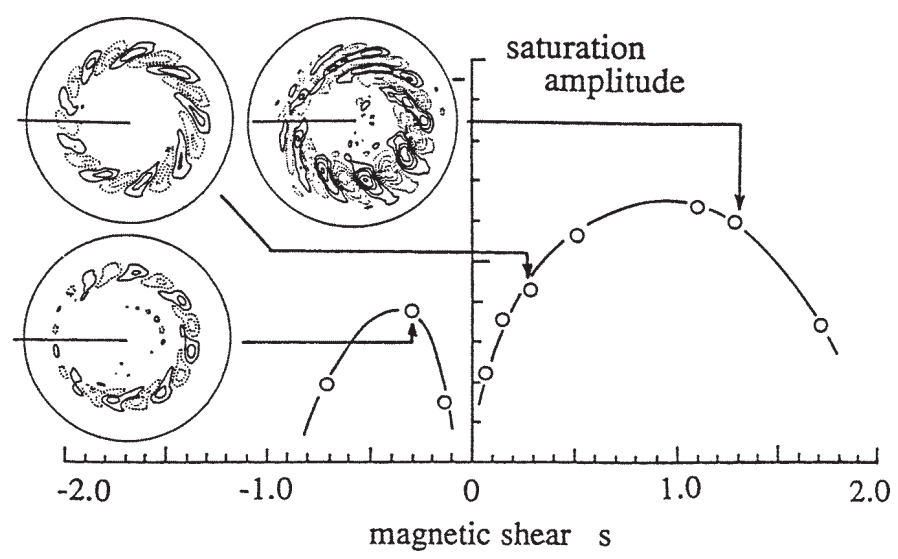

Figure 7. Dependence of the saturation amplitude of electrostatic potential fluctuations on magnetic shear $s=(r / q)(\mathrm{d} q / \mathrm{d} r)$ and potential contours in the poloidal plane for different values of shear (from Wakatani (1998)). In the case shown, the saturation amplitude is proportional to the linear growth rate of the fluctuations. Both for strongly positive magnetic shear $(s>0)$ and negative magnetic shear $(s<0)$ the growth rate decreases, leading to a reduction in the radial transport.
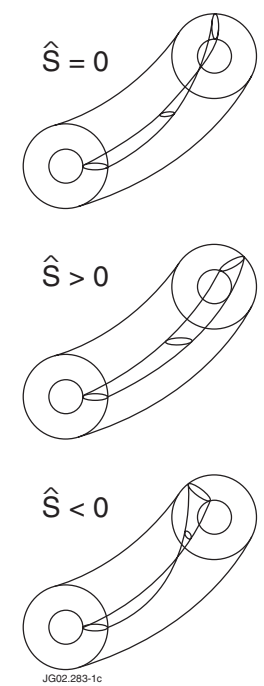

Figure 8. Effect of the magnetic shear $\hat{s}$ on the orientation of the convective cells (diagram reproduced from Antonsen (1996)). The extension in major radius direction is best preserved for $\hat{s}>0$.

preserved. The macroscopic MHD stability follows a similar dependence as shown for the microinstabilities in figure 7 . The stability against ballooning modes allows larger pressure gradients for both high and low magnetic shear. In the latter case access to the second stability region removes the pressure limit due to high- $n$ ballooning modes altogether (see section 4.3).

A different argument regarding the transport reduction due to low magnetic shear is based on the dependence of the turbulence on the spatial density of rational surfaces. The rarefaction of low order rational $q$ values in the vicinity of a $q$ profile minimum is proposed to cause the transport reduction (Garbet et al 2001). This effect is illustrated in figure 9. While for the monotonic $q$ profile the transport is not reduced, the reversed shear cases correspond to 

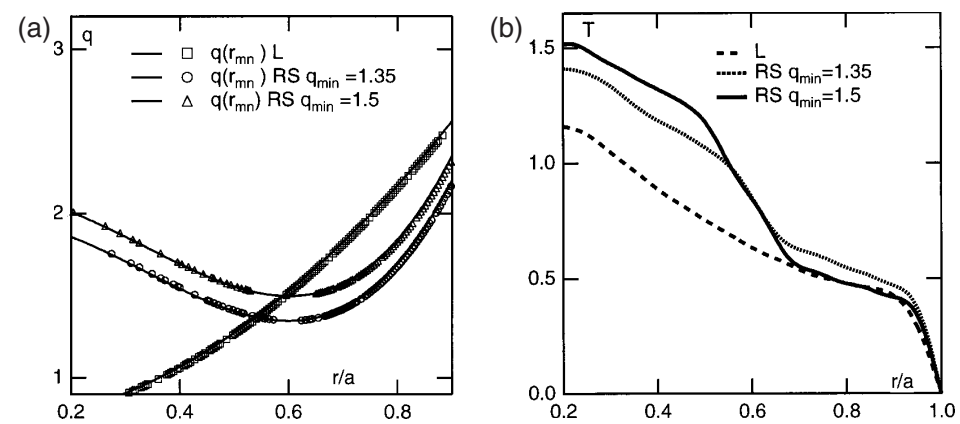

Figure 9. Transport simulations showing the effect of the density of low order rational $q$ values (from Garbet (2001)). In (a) three different $q$ profiles are presented. The rational $q$ values are indicated by the different symbols. For negative magnetic shear the rational $q$ values become sparse at the minimum of the profile, resulting in a steepening of the ion temperature gradient in $(b)$. This region of reduced transport increases with decreasing number of rational surfaces close to the location of the minimum.

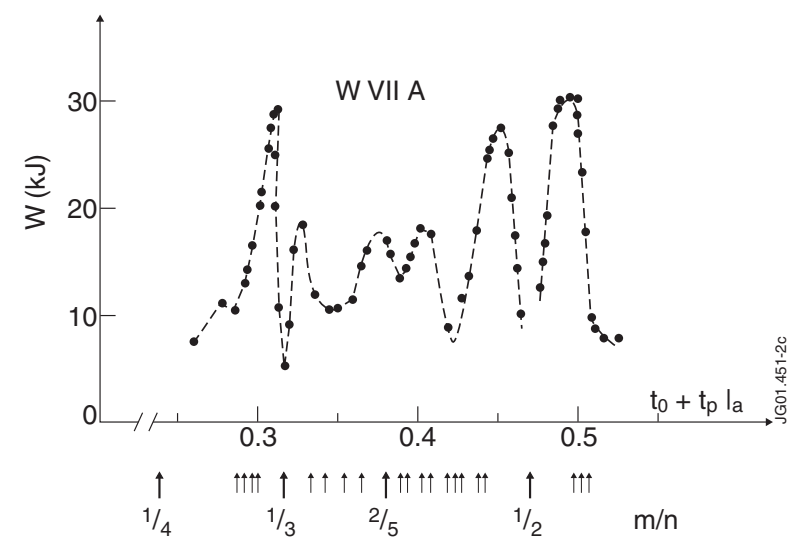

Figure 10. Dependence of the plasma confinement in a low shear stellarator (here Wendelstein VII-A) on the rotational transform $\iota=1 / q$ (reproduced from Wobig (1987)); copyright in these materials is vested in the International Atomic Energy Agency, Vienna, Austria from which permission for publication must be obtained). The confinement is represented by the total plasma energy, which is plotted against the edge $\iota_{a}$. Owing to the bootstrap current, the core $\iota$ deviates slightly from its edge value.

temperature profiles which show a steepening of the gradient in the zero shear region, the extent of which increases with decreasing number of adjacent rational surfaces. This behaviour bears resemblance to the dependence of the confinement on $\iota^{10}$ in low shear stellarators (Wobig et al 1987, Jaenicke et al 1993, Brakel et al 1997). As seen in figure 10 the confinement is worst for $\iota$ equal to low order rational values, $n / m$, and best in their proximity, where narrow zones, depleted of such rationals, are found.

2.2.2. Shafranov shift. The Shafranov shift, which is the displacement of the magnetic flux surfaces with respect to the centre of the bounding surface (Wesson 1997) caused by a rise in the plasma pressure in toroidal geometry, represents an additional turbulence stabilizing

${ }^{10}$ In stellarators, instead of the safety factor $q$, normally the rotational transform $\iota=1 / q$ is used. 
effect (Beer et al 1997, Jenko et al 2000) ( $\alpha$-stabilization). The $\alpha$-stabilization is related to a modification of the magnetic field topology, compressing the flux surfaces on the low field side and stretching the distance between them on the high field side. Although an increase of the density gradient causes an increase of the Shafranov shift, the $\alpha$-stabilization should not be confused with the possible stabilizing influence of density gradients on ITG modes, directly. $\alpha$-stabilization has been suggested as a positive feedback mechanism for confinement transitions (Beer et al 1997), depending on the balance between the drive of the microinstabilities by an increase of the pressure gradient and their reduction by the $\alpha$-stabilization, supported by other stabilizing influences, such as sheared flows and negative magnetic shear.

2.2.3. Electron to ion temperature ratio. As a consequence of the quasi-neutrality condition (see equation (A12)) the ITG mode stability depends on the ratio of $T_{\mathrm{e}} / T_{\mathrm{i}}$ (Romanelli 1989, Garbet et al 1992). Increasing $T_{\mathrm{e}} / T_{\mathrm{i}}$ destabilizes the modes and shifts the critical $R / L_{T_{\mathrm{i}}}$ to smaller values (Kotschenreuther et al 1995). In the context of an $\alpha$-particle heated fusion plasma this is an important issue, as the fast $\alpha$-particles will predominantly heat the electrons, which, moderated by a high plasma density through collisional energy transfer, will result in $T_{\mathrm{e}} \approx T_{\mathrm{i}}$. Thus, with respect to ITG mode driven ion heat transport, a fusion plasma does not lie at the most favourable operating point.

This is in contrast to most present day ITB experiments, which are usually performed at low density, i.e. weakly coupled ions and electrons, and at best achieve $T_{\mathrm{e}} \approx T_{\mathrm{i}}$ by heating electrons and ions separately (Forest et al 1996, Wolf et al 2000, Günter et al 2000b). One exception is the so-called pellet enhance performance (PEP) mode, in which an ITB at high density and $T_{\mathrm{e}} \approx T_{\mathrm{i}}$ is produced transiently, following the injection of frozen deuterium pellets (Tubbing et al 1991) (see section 3).

2.2.4. Effective ion charge. A further plasma quantity influencing plasma transport is the effective ion charge, $Z_{\text {eff }}$. Under certain conditions the ion transport due to ITG modes is reduced with increasing impurity content (Paccagnella et al 1990, Kotschenreuther et al 1995, Tokar et al 1999, Staebler et al 1999). The effect of adding impurities is twofold. They cause an increase of $Z_{\text {eff }}$ and thus modify the electron particle balance, and tend to raise the peaking of the electron density profile. However, depending on the details of the radial distribution of electrons, main plasma ions and impurity ions, impurities can also have the opposite effect, namely an increase of the transport. For flat main ion density profiles, for instance, the stabilization of ITG modes by impurities can turn into destabilization (Fröjdh et al 1992). Besides, while ITG modes may be stabilized by the addition of impurities to the plasma, others may be destabilized (Dominguez and Staebler 1993). The resulting heat or particle transport then depends on the balance between the contributions of the different turbulent modes.

In recent years these effects have been extensively studied in the so-called radiative improved or RI-mode in the limiter tokamak TEXTOR (Messiaen et al 1996, Weynants et al 1999). Similar to earlier experiments in ISX-B (Lazarus et al 1985), the RI-mode features a significant confinement improvement over the L-mode, when seeding small amounts of noble gases at the plasma periphery. Besides the density peaking, a favourable dependence of $\tau_{\mathrm{E}}$ on the plasma density, $\tau_{\mathrm{E}} \propto \bar{n}_{\mathrm{e}}$, is observed, resulting in high confinement at Greenwald density (Greenwald et al 1988). In addition, the large radiated power fraction leads to heat removal, which, in contrast to heat conduction or convection, is uniformly deposited among the wall components. Since most of the radiation is close to the plasma periphery, this takes place without confinement degradation. 
(a)

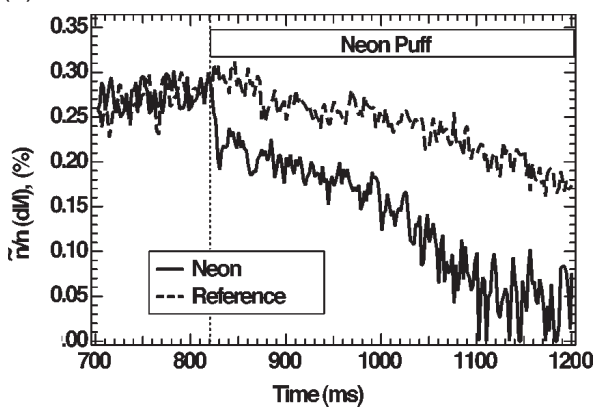

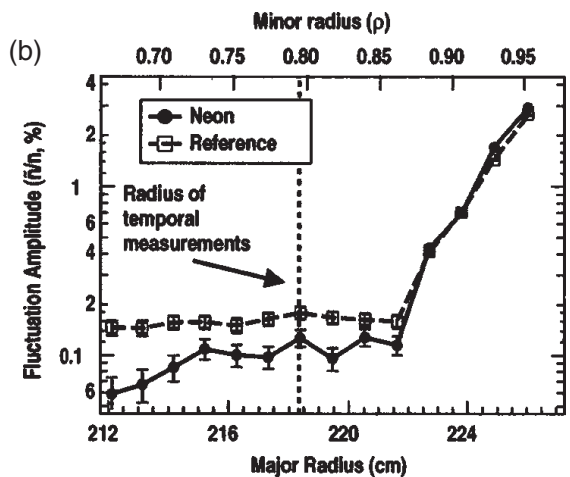

Figure 11. Density fluctuations $\tilde{n} / n$ in the DIII-D tokamak, comparing reversed shear plasmas with (- $)$ and without (- - -) the feeding of neon (neon puff) (from McKee (2000a)). (a) Temporal evolution of $\tilde{n} / n$ at a fixed radius and $(b)$ radial profiles of $\tilde{n} / n$ at $1.1-1.2 \mathrm{~s}$. The density fluctuations have been measured using beam emission spectroscopy (BES) (Fonck et al 1990, Paul and Fonck 1990, McKee et al 1999). The fast initial drop of $\tilde{n} / n$ is explained by the stabilizing effect of impurities on ITG modes directly.

Some of the RI-mode properties have been reproduced in other experiments. In the limiter tokamak TFTR (Hill et al 1999) the main effect of impurity seeding is the increase of the radiated power fraction, while the gain in energy confinement, compared to the reference case without impurity injection, is smaller than in TEXTOR. However, the reference scenario, the so-called supershot regime (Strachan et al 1987), is already above L-mode confinement $\left(\tau_{E} \approx 1.5 \tau_{\text {ITER89-P) }}\right.$. In ASDEX Upgrade (Gruber et al 1995) and DIII-D (Jackson et al 1999) divertor plasmas impurity seeding led to high radiated power fractions and a reduction of the peak heat flux to the divertor target up to complete detachment of the divertor plasma. Although the target load caused by ELMs could be mitigated, while retaining H-mode confinement, these cases do not give clear evidence for an impurity induced turbulence suppression.

At lower density and with a different current profile compared to the RI-mode (negative central shear, i.e. low $l_{\mathrm{i}}$, instead of high $l_{\mathrm{i}}$ ), the effect of impurities on plasma turbulence has been measured directly in DIII-D (McKee et al 2000a, b). Consistent with an increase of the energy confinement time of $80 \%$ and a reduction mainly of the ion heat conductivity, the density fluctuations decrease in the plasma core after neon injection (figure 11). This is explained by the combination of direct effects of the impurity ions on the stability of ITG modes and indirect effects from changes of the pressure and toroidal rotation profiles resulting from the additional radiation.

2.2.5. Comparison of electron and ion transport. The effects described so far mainly concern transport by ITG and trapped electron modes. The associated fluctuations exhibit spatial scales larger than the ion Larmor radius $\left(k_{\theta} \rho_{\mathrm{i}}<1\right.$, with $\rho_{\mathrm{i}} \propto \sqrt{A_{\mathrm{i}} T} / Z_{\mathrm{i}} B$, where $A_{i}$ and $Z_{i}$ denote the ion charge and mass numbers, $T$ the temperature and $B$ the magnetic field of the plasma). The respective counterparts to ITG modes are electron temperature gradient or ETG modes (Drake et al 1988, Horton et al 1988), which may determine the anomalous electron transport if the critical value of $R / L_{T_{\mathrm{e}}}$ is exceeded. Exchanging the ion with the electron mass, the linear dynamics of ETG modes bear strong resemblance to those of ITG modes, however, on much smaller spatial scales $\left(\rho_{\mathrm{e}}=\sqrt{m_{\mathrm{e}} / m_{\mathrm{i}}} \rho_{\mathrm{i}} \approx(1 / 60) \rho_{i}\right.$ for a deuterium plasma) and with correspondingly larger linear growth rates. However, unlike for ITG and TEM, on the spatial scale of the ETG modes the ion density response to potential fluctuations can be regarded as 
adiabatic (Jenko et al 2001) due to finite orbit effects of the much larger ion orbits. Hence, in contrast to the interaction between ITG and TEM, which raises not only the ion but also the electron and particle transport, ETG modes do not affect the ion transport.

Simulations of the linear instability of ETG modes, based on this isomorphism between ITG and ETG turbulence, predict an energy transport which is smaller by $\sqrt{m_{\mathrm{e}} / m_{\mathrm{i}}} \approx 1 / 60$ for the electrons than that for the ions, which is generally not observed in experiments. Experimental values of $\chi_{\mathrm{e}}$ and $\chi_{\mathrm{i}}$ are generally of the same order of magnitude while, on the other hand, strong evidence for $T_{\mathrm{e}}$ profile resilience has been found in electron transport dominated regimes (Ryter et al 2001a,d). Actually, the measured critical $R / L_{T_{\mathrm{e}}}$ values agree rather well over a wide range of tokamak experiments, lying just below 10 (Ryter et al 2001b) and suggesting a common transport mechanism similar to that of the ions. For comparison the critical ion temperature gradient, $R /\left.L_{T_{\mathrm{i}}}\right|_{\text {crit }}$, is lower by a factor of about 1.5-2 (Baker et al 2001, Peeters et al 2001a, Wolf et al 2001b) (see section 2.3.3).

Recent non-linear theoretical simulations of ETG driven turbulence in toroidal geometry (Jenko et al 2000) may resolve this apparent inconsistency. These simulations show the appearance of radially extended structures, so-called streamers, which are larger than the electron Larmor radius and thus may increase the transport to the level caused by ITG modes. This may explain the observed electron heat transport, in particular, in plasmas with low ion temperatures, where the contribution of ITG and trapped electron modes to the overall transport is expected to be low.

Although linear calculations of the ETG turbulence result in transport levels which are too low, it is nevertheless feasible to study the threshold $R /\left.L_{T_{\mathrm{e}}}\right|_{\text {crit }}$ at which ETG modes occur using linear approximations. The inclusion of non-linear effects mainly changes the dependence of the growth rate on $\nabla T_{\mathrm{e}} / T_{\mathrm{e}}$, and accordingly the heat conductivity, and to a lesser extent the threshold itself. Parameter studies of the linear threshold of toroidal ETG modes (Jenko et al 2001) exhibit corresponding dependences as for ITG modes. The dependence on $T_{\mathrm{e}} / T_{\mathrm{i}}$ is reversed. Increasing this ratio is stabilizing, in contrast to ITG modes, for which it is destabilizing. Similarly to ITG turbulence, $R /\left.L_{T_{\mathrm{e}}}\right|_{\text {crit }}$ reduces with decreasing magnetic shear. The non-linear simulations predict a complete suppression of the ETG modes when the magnetic shear approaches zero or becomes negative (Jenko et al 2001). As discussed below, the magnetic shear stabilization of ETG modes is of particular importance for the formation of transport barriers, since sheared flows may not have any effect.

2.2.6. E $\times$ B flow shear stabilization. Magnetic shear stabilization of plasma turbulence is the main experimental tool in present day tokamaks to produce internal transport barriers. An equally important role is played by the stabilization of the turbulent eddies by sheared $\vec{E} \times \vec{B}$ velocities (Burrell 1997 and references therein). In non-linear theory these sheared flows result in a decorrelation of the turbulence and hence in a reduction of the transport (Biglari et al 1990), but not necessarily in a complete suppression of the turbulence. In the linear approach, for sufficiently large sheared flows, the turbulent modes are completely stabilized. Accordingly, turbulence is suppressed if the shearing rate $\omega_{E \times B}$ exceeds the maximum linear growth rate $\gamma_{\max }^{\operatorname{lin}}$ of the dominant or most unstable mode (Waltz et al 1994, 1998).

$$
\omega_{E \times B}>\gamma_{\max }^{\operatorname{lin}} \sim \frac{v_{\mathrm{T}}}{L_{T}} f(s)
$$

Of course, there is a strong link with the other stabilizing mechanisms, in particular the magnetic shear, as they influence the magnitude of the growth rate. In the relation above, this is expressed by $f(s)$, which adds to the principal dependence on the thermal velocity, $v_{\mathrm{T}}$, or the Larmor radius contained therein, and the temperature gradient length, $L_{T}$. Unlike in fluid dynamics, 
where a parallel shear flow $\approx \mathrm{d} v_{\phi} / \mathrm{d} r$ drives the Kelvin-Helmholtz instability, in a tokamak plasma this appears to be compensated by the combined effects of magnetic shear and the corresponding $E \times B$ flow shear $\omega_{E \times B} \approx(r / q)(\mathrm{d} / \mathrm{d} r)\left(v_{\phi} / R\right)$ (Waltz et al 1994).

In an arbitrary shaped finite aspect ratio tokamak equilibrium the $E \times B$ shearing rate depends on the radial derivative of $E_{r} / R B_{\theta}$ and the correlation lengths of the ambient turbulence (Hahm and Burrell 1995). Since the correlation lengths in the various directions are experimentally not accessible, the turbulence is assumed to be isotropic in the plane perpendicular to the magnetic field, which is supported by turbulence simulations (Waltz et al 1994). This leads to the following approximation

$$
\omega_{E \times B} \approx\left|\frac{R B_{\theta}}{B_{\phi}} \frac{\partial}{\partial r}\left(\frac{E_{r}}{R B_{\theta}}\right)\right| .
$$

$B_{\theta}$ and $B_{\phi}$ are the poloidal and toroidal magnetic field components, respectively, and $r$ is the minor radius at the outer midplane of the plasma, where, due to the ballooning character of the turbulent modes, their growth rate is largest.

The radial electric field $E_{r}$ is given by the radial force balance

$$
E_{r}=\frac{1}{Z_{i} e n_{i}} \frac{\mathrm{d} p_{i}}{\mathrm{~d} r}+v_{\phi, i} B_{\theta}-v_{\theta, i} B_{\phi},
$$

which is valid for any plasma species, such as main ions, impurity ions, or electrons. Based on charge exchange recombination spectroscopy (CXRS) measurements of the toroidal $\left(v_{\phi, i}\right)$ and the poloidal $\left(v_{\theta, i}\right)$ rotation velocities and the pressure gradient $\left(\mathrm{d} p_{i} / \mathrm{d} r\right), E_{r}$ can be determined experimentally (Hawkes et al 1992, Bell et al 1999, 1999b, Meister et al 2001). A more detailed discussion concerning $E_{r}$ is given in the appendix (section A.3). However, it is worth noting, that the large discrepancies between the neoclassical and measured values of $v_{\theta, i}$ (Bell et al 1998a) were artificially produced by an effect specific to this measurement of the poloidal rotation (Bell and Synakowski 2000, Ernst et al 2000, Meister et al 2001). Except for large local excursion of $v_{\theta, i}$ prior to the formation of an ITB, observed in TFTR and ASDEX Upgrade (Bell et al 1998a, De Pena-Hempel et al 1998), the correction of this effect results in quite small poloidal rotation velocities, which agree with neoclassical estimates.

Equations (15) and (16) indicate various feedback mechanisms, which may lead to plasma confinement transitions (Burrell 1997 and references therein). On the one hand, $E_{r}$ depends on the perpendicular heat and particle transport, the angular momentum transport and the poloidal flow and, on the other hand, influences the plasma turbulence by changing the transport and subsequently $E_{r}$ itself. Another feedback loop is given by the bootstrap current. Depending on $\mathrm{d} p / \mathrm{d} r$, it reduces $B_{\theta}$ in the plasma core and thereby contributes to $\omega_{E \times B}$ (Forest et al 1996, Synakowski et al 1997a, Hahm et al 1998). Besides, the bootstrap current tends to reduce the magnetic shear and thus $\gamma_{\max }^{\text {lin }}$, provided one is already in the low magnetic shear region, where $\gamma_{\max } \operatorname{lin}_{\text {ancreases with }} s$ (see figure 7).

Unlike for ITG and trapped electron modes, the effect of the $E \times B$ shear flow on ETG modes and the corresponding turbulence is thought to be limited. Owing to the much smaller scale length $\left(\rho_{\mathrm{e}} \propto m_{\mathrm{e}} v_{T_{\mathrm{e}}}=\sqrt{m_{\mathrm{e}} T_{\mathrm{e}}}\right)$ as compared to the ions, the linear growth rate of the ETG modes is much larger (by the factor $\sqrt{m_{\mathrm{i}} / m_{\mathrm{e}}}$ ) than that of ITG modes, or conversely the usually observed $E \times B$ shearing rate is not large enough. All the more, the magnetic shear stabilization is important.

\subsection{Characterization of internal transport barriers}

In section 1 a rather qualitative definition of an internal transport barrier has been presented (see figure 2), basically defining it as a region of reduced radial transport of energy or particles and 
hence increased pressure gradients. In the literature the definition of an ITB is scarcely more precise. Usually, an ITB is postulated if at constant heating power the gradients of temperature or density increase locally above the previously observed level, which corresponds to a local reduction of the heat or particle diffusivities. Below, possible definitions of internal transport barriers are discussed.

2.3.1. Examples of internal transport barriers. An exceptional example of very localized drops of the electron heat conductivity is an empirical model, used to describe electron temperature profiles obtained in electron cyclotron resonance heated plasmas in RTP (Lopes Cardozo et al 1997, de Baar et al 1997,Hogeweij et al 1998, de Baar et al 1999) and recently also in TEXTOR (Schüller et al 2000). Depending on the radial location of the power deposition of the applied electron cyclotron resonance heating (ECRH), which is narrow with respect to the plasma radius, and on certain rational $q$ values being contained in the plasma, the core electron temperature adopts different values with a step-like profile shape (figure 12). This behaviour can be described by an electron heat conductivity, which is a function of $q$ and only at low order rational $q$ values exhibits regions of reduced transport. With respect to $q$ the width of these barriers is constant. Thus, the spatial structure of $\chi_{\mathrm{e}}$ is determined by the momentary shape of the $q$ profile, where the actual radial extent of the barrier is given by the local magnetic shear (figure 12). One possible explanation, suggested by (de Baar et al 1999) for the alternating layers of high and low confinement, is the formation of magnetic island chains at rational $q$-surfaces. However, up to now such a dependence has not been derived from basic plasma physics principles.

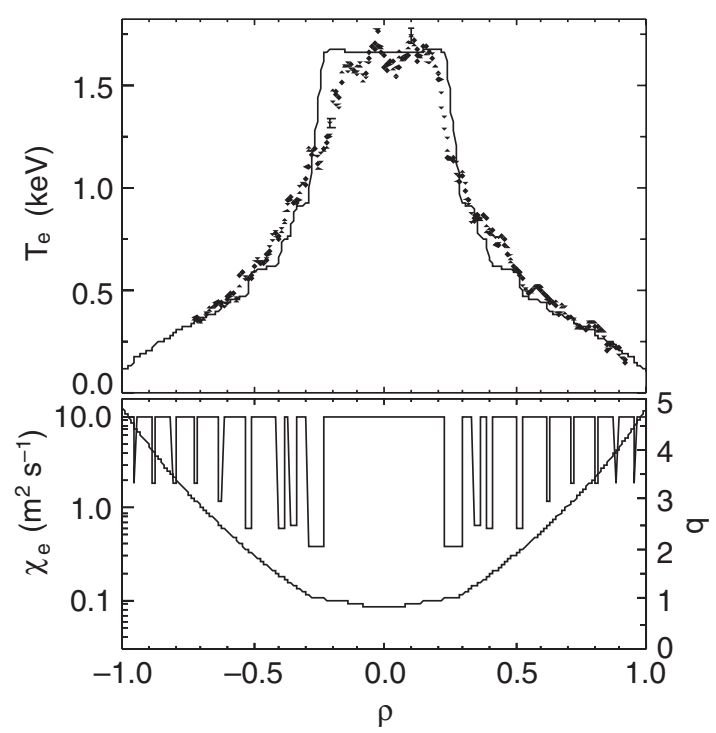

Figure 12. Example of ITBs defined as local incursions of the electron heat conductivity (from Hogeweij et al (1998)); copyright in these materials is vested in the International Atomic Energy Agency, Vienna, Austria from which permission for publication must be obtained). In the top graph experimental electron temperature data, $T_{\mathrm{e}}$, are overlaid with the model calculation (line drawn through). The bottom picture shows the $q$ profile and the spatial structure of the electron heat conductivity, $\chi_{\mathrm{e}}$, which is used to model the $T_{\mathrm{e}}$ profile. The function $\chi_{\mathrm{e}}=f(q)$ is constant except at low order rational values of $q$, where incursions with constant width are introduced. The spatial width of these barriers is then determined by the local magnetic shear. 


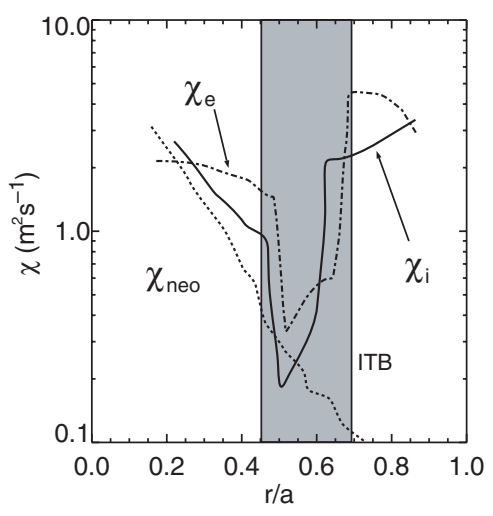

Figure 13. Example of an internal transport barrier (shaded region) in the JT-60U tokamak, becoming evident as a localized drop of the ion and electron thermal conductivities $\left(\chi_{\mathrm{i}}\right.$ and $\left.\chi_{\mathrm{e}}\right)$ which are plotted against the normalized plasma radius (reproduced from Shirai et al (1999)); copyright in these materials is vested in the International Atomic Energy Agency, Vienna, Austria from which permission for publication must be obtained). $\chi_{i}$ and $\chi_{\mathrm{e}}$ decrease by factors of 10 to 20 within $5 \mathrm{~cm}$. Also shown is the calculated neoclassical ion heat conductivity, $\chi_{\text {neo }}$

A different approach to explain the steep temperature gradients, in particular at the $q=1$ surface has been proposed by (Porcelli et al 1999). Instead of assuming reduced perpendicular heat diffusion near the $q=1$ surface, the transport barrier is a consequence of the heat convection, caused by sawtooth relaxation oscillations. This mechanism can reproduce the electron temperature profiles even with a constant electron heat conductivity, provided the growth time of the magnetic island is shorter than the cross-field heat diffusion time and the width of the electron cyclotron power deposition is smaller than the $q=1$ radius. As a radial variation of the heat diffusion is not involved, one would not necessarily speak of a transport barrier in this case. However, it is still a matter of controversy which of the two models better suits the experimental results (Beurskens et al 2001).

A more conventional case of an internal transport barrier in terms of a single localized reduction of the transport coefficients is shown in figure 13 (Fujita et al 1997a). According to the connection between the heat flux $Q$ and the heat conductivity $\chi$,

$$
Q=-\chi n \nabla T=-\chi p \frac{\nabla T}{T}
$$

such localized drops of $\chi$ correspond to sharp gradients of the temperature and density profiles, illustrated in figure 14 (Doyle et al 2000). As shown for the examples from the tokamaks JT60U and DIII-D, such clear internal transport barriers are typically produced in plasmas with negative central magnetic shear, where they are located in the negative shear region close to the minimum of the $q$ profile. Besides the magnetic shear stabilization, the momentum transfer from the neutral heating beams, increasing the $E \times B$ shearing rate, plays a decisive role in reducing the turbulence in these plasmas.

2.3.2. Transport at neoclassical level. Within the accuracy of theory and measurements, the thermal ion and particle diffusivities are often reduced to their neoclassical levels in the region of the internal transport barrier, while $\chi_{\mathrm{e}} \approx \chi_{\mathrm{i}}$ still remains significantly above the neoclassical electron transport. Considering the ion transport only, two experimental observations require revisions of theoretical predictions regarding neoclassical transport or their applicability in plasmas with low or even negative magnetic shear. First, ITBs with $\chi$ lower than the standard 

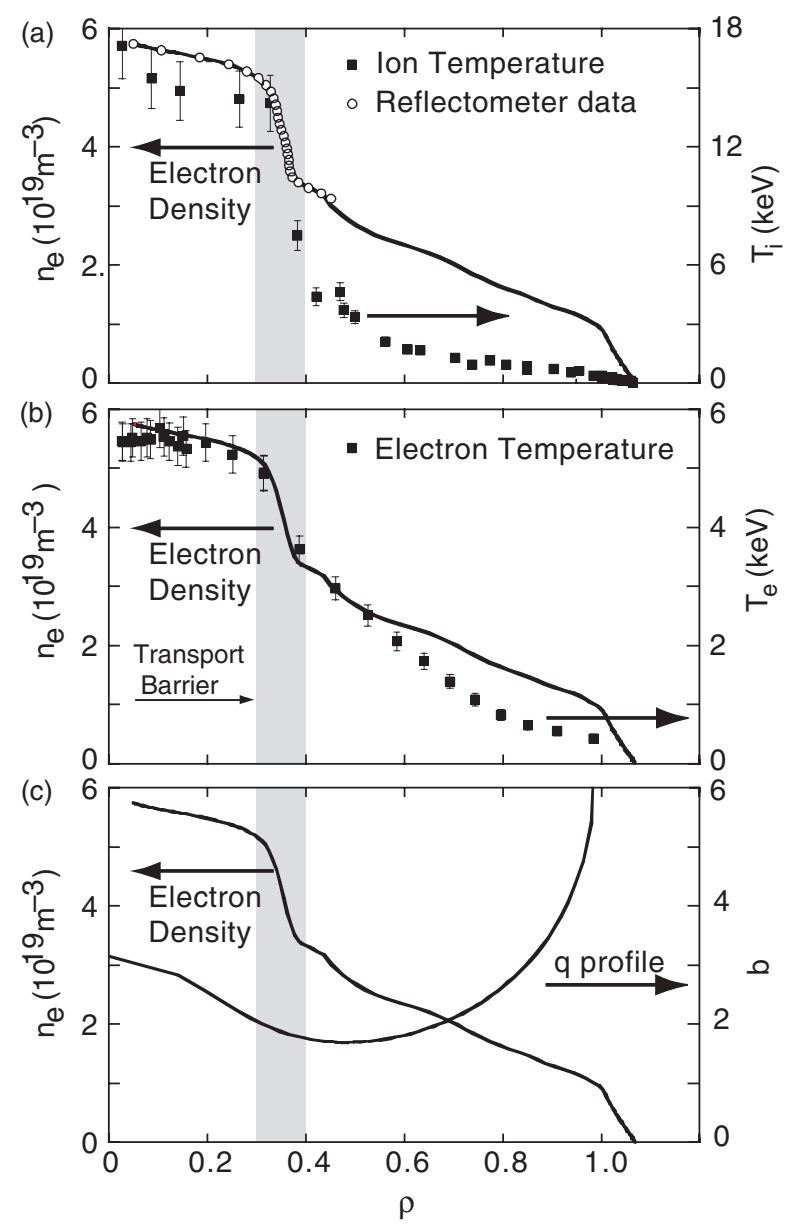

Figure 14. Example of an internal transport barrier (shaded region) in the presence of negative central shear in the DIII-D tokamak (reproduced from Doyle (2000)). Shown are the profiles of ion temperature $(a)$ electron temperature $(b)$ and $q$ profile $(c)$. The good spatial correlation of particle, electron and ion thermal barriers becomes apparent by the superposition with the electron density profile.

neoclassical predictions (Chang and Hinton 1986) have been observed (Levinton et al 1995). It should, however, not be possible to fall below the collisionally induced neoclassical transport. Second, the flattening of the profiles inside the ITB (figure 14), not seen in conventional confinement regimes such as the H-mode, corresponds to a strong increase of the radial transport up to a complete loss of confinement for temperature gradients approaching zero. In general, this is not well described by neoclassical theory.

Standard neoclassical theory assumes the radial size of the particle orbits to be small compared to the local minor radius and the pressure gradient length, $L_{p}=p / \nabla p$. Approaching the magnetic axis, this approximation becomes invalid. The radial extent of this region is determined by the size of the trapped particle orbits, which is given by (Peeters et al 2001a)

$$
r_{\text {orbit }} \propto(q \rho)^{2 / 3} R^{1 / 3},
$$

where $q$ is the safety factor, $\rho$ the Larmor radius and $R$ the major radius. Consequently, in plasmas with low or negative central shear, corresponding to a large central $q$ or low 
current density and poloidal magnetic field, the orbits increase, extending the region where the assumptions made in standard neoclassical theory are violated, to larger radii.

However, the different analytical theories (Shaing et al 1997, Lin et al 1997a), which include the effects of finite orbit widths, also contradict each other. While Lin et al (1997a) predict a low central $\chi_{\mathrm{i}}$ even in the presence of shear reversal, the $\chi_{i}$ from Shaing et al (1997) increases towards the plasma centre (figure 15). Another approach is to use Monte-Carlo techniques to numerically evaluate the neoclassical ion transport (Lin et al 1995, Bergmann et al 2001). In figure 15 such Monte-Carlo calculations for an ASDEX Upgrade reversed magnetic shear ITB are compared to the corresponding results from Lin et al (1997a) and Shaing et al (1997). Similar to ITBs in TFTR (Lin et al 1997b), the best agreement with the measurement in the inner half of the plasma is achieved by the Monte-Carlo technique, both, in reproducing the large drop of $\chi_{\mathrm{i}}$ in the region of the transport barrier and its increase associated with the often observed temperature plateaus close to the magnetic axis. In the example shown the analytical descriptions which include the finite orbit effects either under- or overestimate the ion heat transport.

Another effect which may influence the neoclassical transport is the orbit squeezing associated with the shear of the radial electric field (Hazeltine 1989, Wakatani 1998). The same shearing of $E_{r}$ which is thought to be responsible for the decorrelation of the turbulent eddies may result in a reduction of the neoclassical ion thermal transport near the magnetic axis of the plasma.

From the discussion above it becomes clear, that it is not feasible to define internal barriers of the thermal ion transport as regions where the transport is at the neoclassical level, since both the theoretically and experimentally determined transport coefficients are not known to an accurate enough level. A major uncertainty of the experimental values comes from the determination of the heat flux in the plasma, which is usually inferred very indirectly from

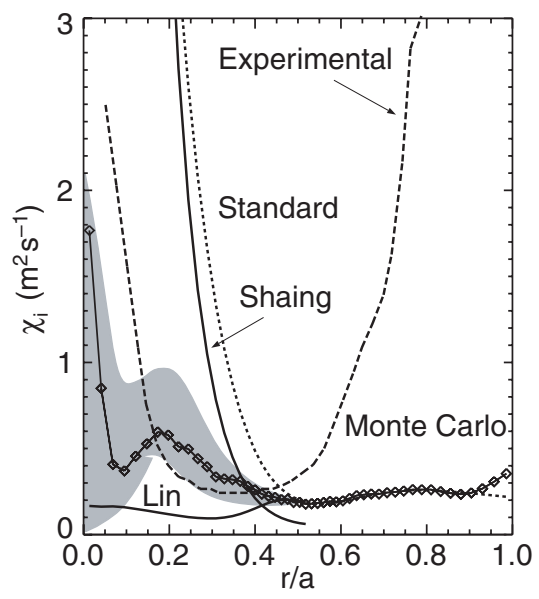

Figure 15. The numerically evaluated neoclassical ion heat conductivity using Monte-Carlo techniques is compared to standard neoclassical theory, neglecting finite orbit width effects, and analytical calculations (Shaing et al 1997, Lin et al 1997a), which, although they both include finite orbit effects, contradict each other (from Peeters et al (2001a); copyright in these materials is vested in the International Atomic Energy Agency, Vienna, Austria from which permission for publication must be obtained). The underlying plasma parameters are taken from an ASDEX Upgrade discharge with negative central shear $\left(q_{0} \approx 4\right.$ and $\left.q_{\min } \geqslant 2\right)$ and an ITB with central values of $T_{\mathrm{i}}=15 \mathrm{keV}$ and $T_{\mathrm{e}}=5 \mathrm{keV}$. The $\chi_{\mathrm{i}}$, inferred from the measured temperature and density profiles, shows the best agreement with the Monte-Carlo calculations. 
calculations of the power deposition of the various heat sources. In addition, such a definition cannot be applied to the electron transport. Although in plasmas with ion transport barriers similar reductions of the electron gradient length are often observed (Forest et al 1996,Litaudon et al 1999, Shirai et al 1999, Doyle et al 2000, Wolf et al 2000) (see figure 14), the electron transport is generally far above neoclassical (Rice et al 1996a, Baranov et al 1999, Greenfield et al 1999, Shirai et al 1999, Becoulet et al 2000, Wolf et al 2000) (see figure 13), which is predicted to be lower than the ion heat transport by a factor of $\sqrt{m_{\mathrm{e}} / m_{\mathrm{i}}}$.

According to equation (17) the temperature plateaus often seen in the centre of ITB plasmas imply very poor central confinement. For the ions this is at least qualitatively in agreement with the influence of the increase of the trapped particle orbit widths (equation (18)). However, for the electrons with their much smaller Larmor radii this cannot serve as an explanation for such an increase of the radial transport. Also, postulating a large anomalous contribution does not resolve this contradiction, since for $\nabla T_{\mathrm{e}}$ approaching zero the drive of the turbulence is missing. Only if the poloidal magnetic field was zero over an extended region close to the magnetic axis ${ }^{11}$, a breakdown of the MHD equilibrium, as $\iota=1 / q$ becomes zero, could readily explain this loss of central confinement. While, in general, plasmas with reversed magnetic shear exhibit finite central $q$ values (Rice et al 1996c, Wolf et al 2001a) and nevertheless show rather flat electron temperature profiles (Rice et al 1996b, Wolf et al 2001a), the extreme case of $q_{0}$ approaching infinity has been observed in JET (Hawkes et al 2001) and JT-60U (Fujita et al 2001a). Owing to strong off-axis current drive and electron heating during the ramp-up phase of the discharge, the combination of a slow radial current diffusion in a highly conductive plasma and sufficiently strong external current drive results in the current density becoming zero in the vicinity of the magnetic axis, which can be sustained for several seconds (figure 16). Therefore, these plasma conditions are also referred to as "current hole".

Other possible explanations for the electron transport being above the neoclassical level even in the presence of ITBs could include the effect of small scale perturbations of the magnetic field. Due to the smaller Larmor radius of the electrons and their much higher velocity along the field lines as compared to the ions, electrons are more susceptible to perturbations of the magnetic field topology. If a magnetic island has a width of the order of or below the ion Larmor radius, ions would not be affected very much, while cross-field diffusion would be enhanced for electrons.

2.3.3. Approaches to define internal transport barriers. Assuming that in conventional L-and $\mathrm{H}$-mode plasmas the profiles of ion and electron temperatures are stiff, ITBs can be defined as a reduction of $L_{T}$ with respect to the critical value, $\left.L_{T}\right|_{\text {crit }}$, of the conventional plasmas (Peeters et al 2001a, b). This is illustrated in figure 17. In contrast to the stiff profiles of L- and $\mathrm{H}$-mode (see also figure 6), the ITB, here of the ion temperature, clearly shows a $L_{T_{\mathrm{i}}}=T_{\mathrm{i}} / \nabla T_{\mathrm{i}}$ at half radius which is lower by a factor of about three. While the plasma edge remains L-mode like, an ITB develops in the core region where the magnetic shear is negative, owing to early heating of the plasma in the current ramp. The heating power in all three cases (L-, H-mode and ITB) is the same.

According to equation (12) in section 2.1 the ratio $T(\rho) / T\left(\rho_{\mathrm{b}}\right)$ at any two radii is constant, if the radial distribution of the gradient length does not change with plasma conditions. In fact, as can be seen in figure 17, $L_{T}$ is even constant over a wide range of the plasma cross section. The distinction between stiff and non-stiff temperature profiles can be visualized by plotting $T(\rho)$ against $T\left(\rho_{\mathrm{b}}\right)$. As shown in figure 18 for the ion temperature profiles of a large number

11 The poloidal magnetic field in a tokamak is generally zero only at the magnetic axis, but with a finite slope corresponding to a finite central current density or safety factor $q$. In contrast, $\mathrm{d} B_{\theta} / \mathrm{d} r=0$ at the magnetic axis implies $\iota=1 / q=0$. 


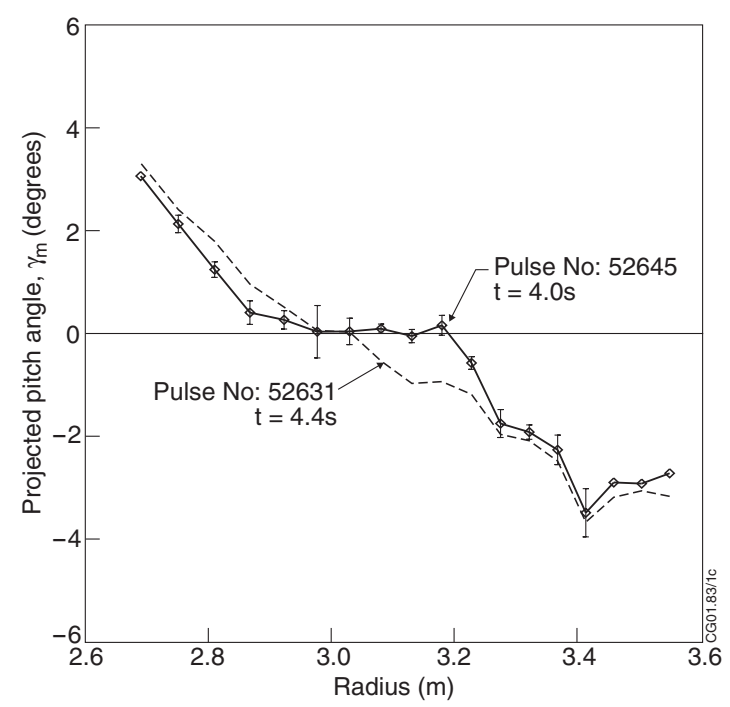

Figure 16. Comparison of the measured MSE polarization angles, $\gamma_{\mathrm{m}}$, projected onto the magnetic midplane for two different JET plasmas (from Hawkes et al (2001)). As $\gamma_{\mathrm{m}} \propto B_{\theta}$, the extended region of $\mathrm{d} \gamma_{\mathrm{m}} / \mathrm{d} r=0$ means that there $\iota=1 / q=0(-)$. Hence a MHD equilibrium which confines the plasma does not exist in this region. In contrast, $\mathrm{d} \gamma_{\mathrm{m}} / \mathrm{d} r<0(---)$ corresponds to a finite central $q$, usually present in tokamak plasmas.

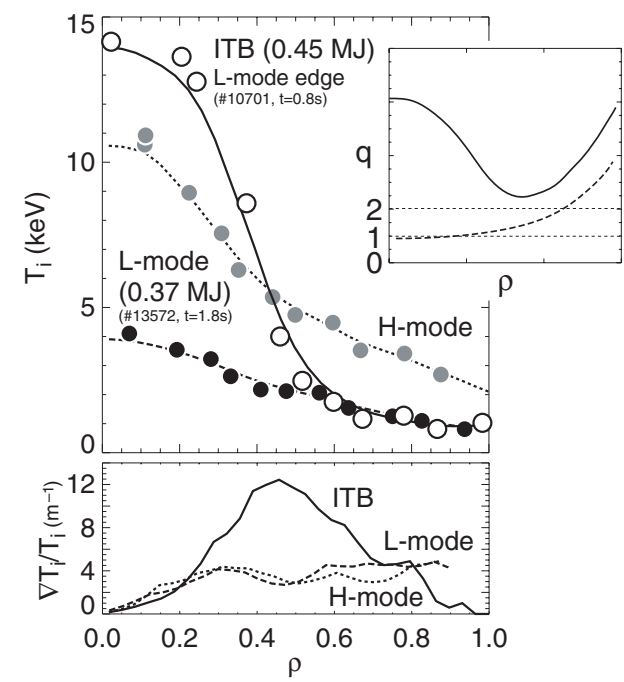

Figure 17. As an extension of figure 6 an ASDEX Upgrade ion temperature profile of an ITB discharge is shown. As the plasma is limited at the inner wall of the torus, the edge remains L-mode like, while due to negative magnetic shear in the plasma centre an ITB develops with $\nabla T_{\mathrm{i}} / T_{\mathrm{i}}$ clearly exceeding the value of the $\mathrm{L}$ - and $\mathrm{H}$-mode cases. The insertion shows the $q$ profiles of the L-mode (- - - ) and the ITB plasma (- - . In the latter case early neutral beam heating in the current ramp is applied, to produce the magnetic shear reversal. At the times when the profiles have been recorded the heating power in all three discharges is the same (5 MW). 

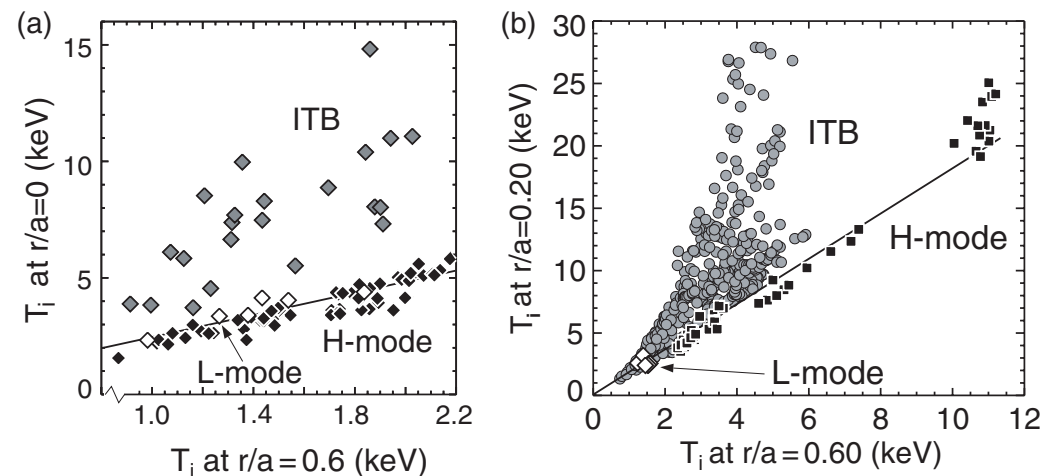

Figure 18. Core versus boundary ion temperatures for (a) ASDEX Upgrade (reproduced from Peeters et al (2001a); copyright in these materials is vested in the International Atomic Energy Agency, Vienna, Austria from which permission for publication must be obtained) and (b) JET (reproduced from Wolf et al (2001b)). The stiff temperature profiles in L- and H-mode follow straight lines in both devices, corresponding to the same $R / L_{T_{\mathrm{i}}}$. The internal transport barriers with their shorter $L_{T_{\mathrm{i}}}$ fill up the space above. The two radial points at which the temperatures are taken have to be chosen such that they embrace the region of reduced $L_{T}$. Hence the data points represent only ITBs which are not broader than $r / a=0.6$.

of ASDEX Upgrade (Peeters et al 2001a, b) and JET discharges (Wolf et al 2001b), all L- and $\mathrm{H}$-mode points follow a straight line. In general the $\mathrm{H}$-mode plasmas exhibit higher values of $T_{\mathrm{i}}(\rho)$ and $T_{\mathrm{i}}\left(\rho_{\mathrm{b}}\right)$, since the higher heating power and the confinement transition at the plasma edge increase $T_{\mathrm{i}}\left(\rho_{\mathrm{b}}\right)$. In contrast, the internal transport barrier cases fill up the space above, corresponding to shorter gradient lengths. Of course, the two radial points have to be chosen such that they embrace the region of reduced $L_{T}$. Correcting for the slightly different aspect ratios of the two devices, which also enter into equation (12), the slopes of the lines representing the stiff temperature profiles give approximately the same $R / L_{T_{\mathrm{i}}}$ in ASDEX Upgrade and JET, further supporting the conjecture of a common transport mechanism in tokamak plasmas.

As discussed in section 2.2, the saturation amplitude or, in linear theory, the growth rate of temperature gradient modes depend on various plasma parameters, including $Z_{\mathrm{eff}}, T_{\mathrm{e}} / T_{\mathrm{i}}$ and magnetic shear. Consequently, the rather small scatter of the L- and H-mode data in figure 18, compared to the deviations observed for the ITB cases, suggests similar turbulence determining parameters in the temperature range shown, although the trend towards a larger $T_{\mathrm{i}}(\rho=0.2) / T_{\mathrm{i}}\left(\rho_{\mathrm{b}}=0.6\right)$ for JET around $20 \mathrm{keV}$ may indicate the increasing stabilization of the ITG modes at a lower $T_{\mathrm{e}} / T_{\mathrm{i}}$ or higher plasma pressure. That, indeed, the dependence of $T_{\mathrm{i}}(\rho)$ on $T_{\mathrm{i}}\left(\rho_{\mathrm{b}}\right)$ does not have to be necessarily linear, in particular if a large temperature range is covered, is illustrated in figure 19. Shown are $T_{\mathrm{i}}(\rho) / T_{\mathrm{i}}\left(\rho_{\mathrm{b}}\right)$ curves, based on theoretical calculations of the ITG mode driven transport (Ernst et al 1998a, Bell et al 1999a). Already, without the turbulence stabilizing effect of the $E \times B$ shear flow the dependence of $T_{\mathrm{i}}(\rho)$ on $T_{\mathrm{i}}\left(\rho_{\mathrm{b}}\right)$ is slightly non-linear. Including the $E \times B$ shearing this non-linearity increases considerably, which is explained by the rise of the pressure gradient at higher temperatures causing an increase of the $E \times B$ shearing rate.

While in the L- and H-mode plasmas all $q$ profiles have a monotonic shape allowing the local magnetic shear to vary only little in the core confinement region, ITB plasmas cover a magnetic shear range from weakly positive to negative. Thus, the statement above has to be relativized. A deviation from the $R / L_{T}=$ const. curve of the $\mathrm{L}$ - and $\mathrm{H}$-mode plasmas may not necessarily mean that such a profile is non-stiff. It could as well be merely a reduction of $L_{T}$, 


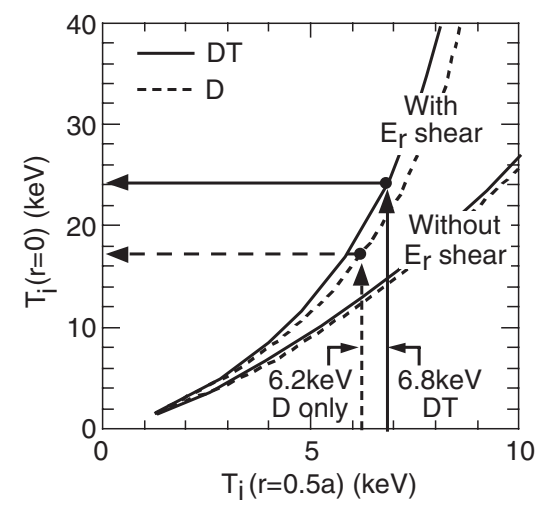

Figure 19. Calculated core versus half-radius ion temperatures for TFTR plasma parameters, including turbulence suppression by self-consistent plasma flows (Ernst et al 1998a) (reproduced from Bell et al (1999a)). The highly non-linear behaviour is caused by the presence of flow shear (with $E_{r}$ shear), which is generated by the plasma pressure gradient itself and has a stabilizing effect on the ITG mode driven turbulence. Also shown is the effect of a modification of the isotope composition, comparing pure deuterium (D) with deuterium tritium (DT) plasmas.

owing to a reduction of, for instance, the magnetic shear, but retaining the profile stiffness at a lower value of $L_{T}$. This difference is illustrated in figure 20. The time trajectory of the plasma with the monotonic $q$ profile (Joffrin et al 2002b) first coincides with the other L- and H-mode plasmas until it makes a transition into the ITB region. As the stabilization of the ITG modes by low magnetic shear can be considered weak, the most likely candidate for triggering this transition is an increase of the $E \times B$ flow shear. In contrast, the negative magnetic shear case deviates even at low temperatures from the L- and $\mathrm{H}$-mode plasmas and later makes a transition to an even lower $L_{T}$, possibly caused by a combination of magnetic and $E \times B$ shear stabilization. Whether one would speak of an ITB before this second transition depends on whether an ITB is regarded as a region where the profile stiffness has to be broken or whether it is sufficient to increase $R /\left.L_{T}\right|_{\text {crit }}$ above the value observed in L- and $\mathrm{H}$-mode plasmas. As discussed above, other effects causing such variations of $R /\left.L_{T}\right|_{\text {crit }}$ include changes of $T_{\mathrm{e}} / T_{\mathrm{i}}$ or $\omega_{E \times B}$.

In conclusion, ITBs of the ion or electron thermal transport can be defined as regions where the gradient length is reduced with respect to the $\mathrm{L}$ - and $\mathrm{H}$-mode values. There is evidence that the latter are governed by temperature gradient modes, producing profile stiffness. Whether or under which conditions the temperature profiles in ITB plasmas remain critical gradient limited is still an open question. Various possibilities include the reduction of the transport to neoclassical level, and smaller reductions with and without the preservation of critical gradients. For the electron transport, the first possibility seems unlikely, as even in the presence of ITBs neoclassical electron transport could not be achieved up to now (Rice et al 1996a, Baranov et al 1999, Greenfield et al 1999, Shirai et al 1999, Becoulet et al 2000, Wolf et al 2000). Of course, also MHD instabilities driven by the large pressure gradients of the ITBs can constitute an upper limit to the temperature gradients (see section 4.3).

Although the data presented in figures 18 and 20 refer to the ion temperature profiles only, similar observations have been made also for the electrons (Ryter et al 2001b, Peeters et al 2001b). In fact, so far the electron transport has been investigated much more thoroughly, since, in contrast to the ions, local heat modulation techniques exist, which allow the propagation of heat waves to be studied (Lopez Cardozo 1995) (see section 2.1).

Based on the condition for stabilization of turbulent modes, given by $\omega_{E \times B}>\gamma_{\max }^{\text {lin }}$ (section 2.2.6), an alternative criterion for the occurrence of ITBs has been proposed by Tresset 


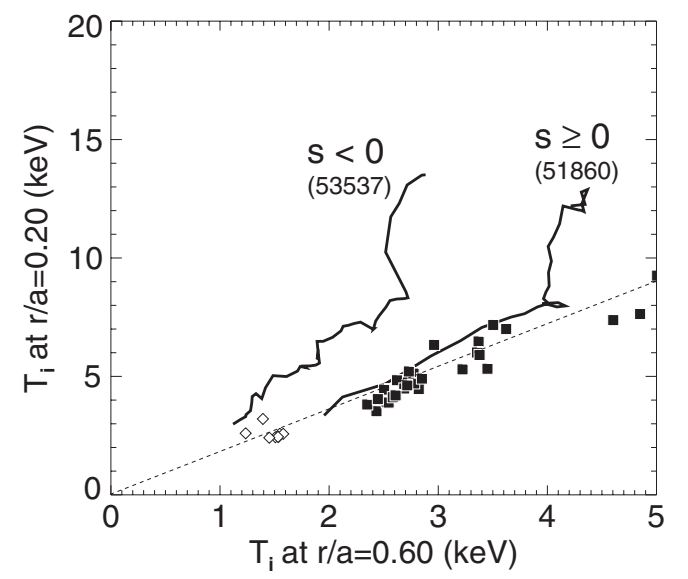

Figure 20. Core versus boundary ion temperatures for the time trajectories of two different JET discharges. The discharge with the monotonic $q$ profile $(s \geqslant 0)$ first follows the L- and H-mode curve and later develops an ITB when the $q=1$ magnetic surface enters the plasma (Joffrin et al $2002 \mathrm{a}, \mathrm{b})$. In contrast, the discharge with the negative central shear $(s<0)$ immediately deviates from the L-and $\mathrm{H}$-mode plasmas and later makes a transition to an even lower $L_{T}$ when the $q=2$ surface appears. This indicates that only in the latter case significant magnetic shear stabilization of the turbulent modes takes place, resulting in a lower $L_{T}$ from the beginning of the discharge.

et al (2001, 2002). Assuming that the magnetic shear stabilization is always the same and that the $E \times B$ shearing rate is dominated by the pressure gradient, an ITB exists if

$$
\rho_{T}^{*}=\frac{\rho_{\mathrm{s}}}{L_{T_{\mathrm{i}, \mathrm{e}}}} \geqslant \rho_{\mathrm{ITB}}^{*},
$$

where

$$
\rho_{T}^{*}=\frac{\rho_{\mathrm{s}}}{L_{T}}=\frac{\sqrt{m_{\mathrm{i}}}}{Z e} \frac{\sqrt{T_{\mathrm{e}}}}{B_{\phi}} \frac{\partial T}{T \partial r}
$$

and $\rho_{\mathrm{ITB}}^{*}$ is an experimentally determined threshold value of the normalized Larmor radius $\rho_{T}^{*}$. A statistical analysis of a large number of JET ITB discharges yielded $\rho_{\text {ITB }}^{*}=1.4 \times 10^{-2}$ (Tresset et al 2001, 2002). Basically expression (19) means that, if the temperature gradient length $L_{T}$ becomes smaller than the turbulence scale length $\rho_{\mathrm{s}}$, it would indicate the suppression of the turbulence. For electron transport by TEMs the scale length is approximated by the Larmor radius at the sound speed, $\rho_{\mathrm{s}} \propto \sqrt{T_{\mathrm{e}} m_{\mathrm{i}}}$. For the ion transport by ITG modes this has to be replaced by the ion Larmor radius, i.e. exchanging $T_{\mathrm{e}}$ against $T_{\mathrm{i}}$ in equation (20). As mentioned in section 2.2.6, ETG modes with their much smaller scale length $\propto \rho_{\mathrm{e}}$ cannot be treated in the same way.

This first attempt to infer a quantitative ITB criterion was mainly motivated by the toroidal magnetic field dependence of the threshold power, required to obtain ITBs in JET plasmas with low but positive magnetic shear (Gormezano 1999, Challis et al 2001a). In the ITB criterion, given by expression (19), the magnetic field dependence is contained in the normalized Larmor radius, $\rho_{T}^{*}$. Figure 21 shows that this ITB criterion, despite the simplifications made, describes the JET data rather well.

Two definitions of ITBs have been presented. In the first, an ITB is characterized by a drop of $L_{T}$ below the critical value, given by stiff temperature profiles, or $R / L_{T}>R /\left.L_{T}\right|_{\text {crit }}$. The second one (expression (20)) is derived from the requirement of a sufficiently high $E \times B$ shearing rate, exceeding the maximum linear growth rate of the dominant microinstability. 


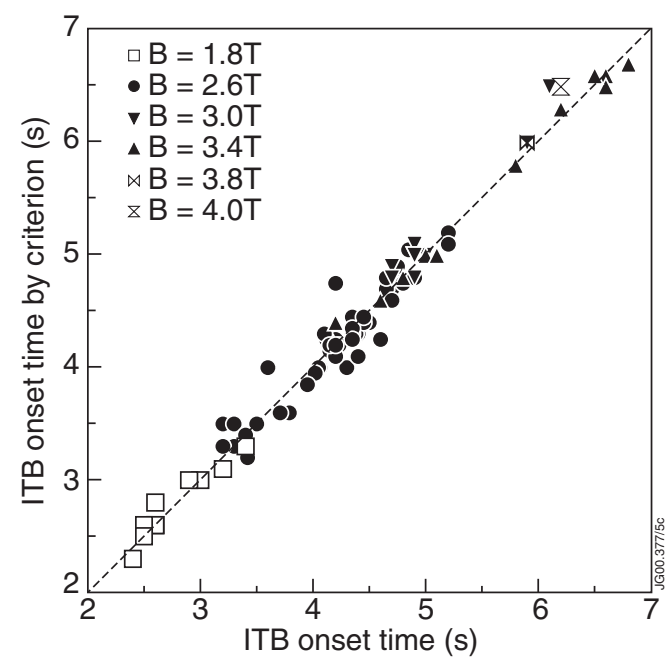

Figure 21. The time, when the ITB emerges according to local changes of the temperature gradients, is compared to the time, detected by the ITB criterion (expression (20)) for a large variety of JET discharges (from Tresset et al (2002); copyright in these materials is vested in the International Atomic Energy Agency, Vienna, Austria from which permission for publication must be obtained). Noticeable is the good agreement for the different toroidal magnetic field values, ranging from 1.8 to $4 \mathrm{~T}$.

This criterion also contains the temperature gradient length as one of the crucial parameters. The main difference, however, between both definitions is, besides the magnetic field dependence, the additional $\sqrt{T}$ term from the normalization to $\rho_{\mathrm{s}}$ in the $\rho^{*}$ criterion, which can lead to contradicting judgements as to whether an ITB is present. It is possible to find two stiff temperature profiles, by definition having the same $L_{T}$, which however lie above and below the $\rho_{\text {ITB }}^{*}$ ITB threshold, as they differ in their absolute temperature values (Peeters et al 2001b). Considering the approximations made, in particular the neglect of the shear of the toroidal plasma rotation and the magnetic shear dependence, which should enter in $\rho_{\mathrm{ITB}}^{*}$ and $R /\left.L_{T}\right|_{\text {crit }}$, further refinements are certainly required.

\section{Survey of plasma regimes with internal transport barriers}

Historically, the beneficial effect of a reduction of the magnetic shear on the transport in tokamak plasmas was already recognized more than thirty years ago (Kadomtsev and Pogutse 1967). However, it took several decades for the first internal transport barriers to be observed, to some extent caused by the inability to produce the desired current profile lacking the necessary techniques.

To date, the standard method to generate low or negative magnetic shear in the plasma centre is to utilize the skin current profile, which forms early in a tokamak discharge when the plasma current builds up. Applying electron heating by external means during this phase, in addition to the generally less efficient Ohmic heating by the plasma current itself, the conductivity $\sigma \propto T_{\mathrm{e}}^{3 / 2}$ is increased and thus the current diffusion process slows down. This preserves the hollow shape of the initial current profile for time scales of the order of the skin time $\tau_{\text {skin }} \sim \sigma(\varepsilon R)^{2}$ ( $R$ is the major radius and $\varepsilon=a / R$ the inverse aspect ratio of the torus). The strong dependence on the size of the tokamak favours the more recently available larger machines in achieving higher values of $\tau_{\mathrm{skin}} / \tau_{\mathrm{E}}$, necessary to study the effects of current profile 
modifications on confinement. As both the current ramp assisted modification of the current profile and its sustainment requires high electron temperatures, the average plasma density has to be kept low enough to achieve the necessary $T_{\mathrm{e}}$ at a given heating power.

Figure 22 gives an overview of the different $q$ profiles obtained in JET using different heating or current drive methods during the current ramp. Other methods to obtain reversed magnetic shear without the use of current ramps include off-axis current drive in the cocurrent direction (Litaudon et al 1996) and on-axis current drive in the counter-current direction (Pietrzyk et al 2001, Wolf et al 2001a). However, here too, low density is required for high current drive efficiencies, approximately scaling like $T_{\mathrm{e}} / n_{\mathrm{e}}=p_{\mathrm{e}} / n_{\mathrm{e}}^{2}$.

Besides the advantage of larger devices in achieving the current profile modification necessary for the confinement improvement, the progress made in plasma control and diagnostic capabilities has also added to the development of tokamak plasmas with ITBs. The improvements in tailoring the current profile without being impeded by detrimental MHD instabilities have been supported, in particular, by the development of current density profile diagnostics (Soltwisch 1992).

The term internal transport barrier was first introduced by Koide et al (1994), while the first internal transport barriers were probably observed following the injection of frozen deuterium pellets in JET (JET Team (presented by G L Schmidt) 1989), providing a strong source of central particle fuelling. This PEP mode was developed in JET (Tubbing et al 1991, Hugon et al 1992) and subsequently also investigated in many other tokamaks (Kamada et al 1991, Geraud

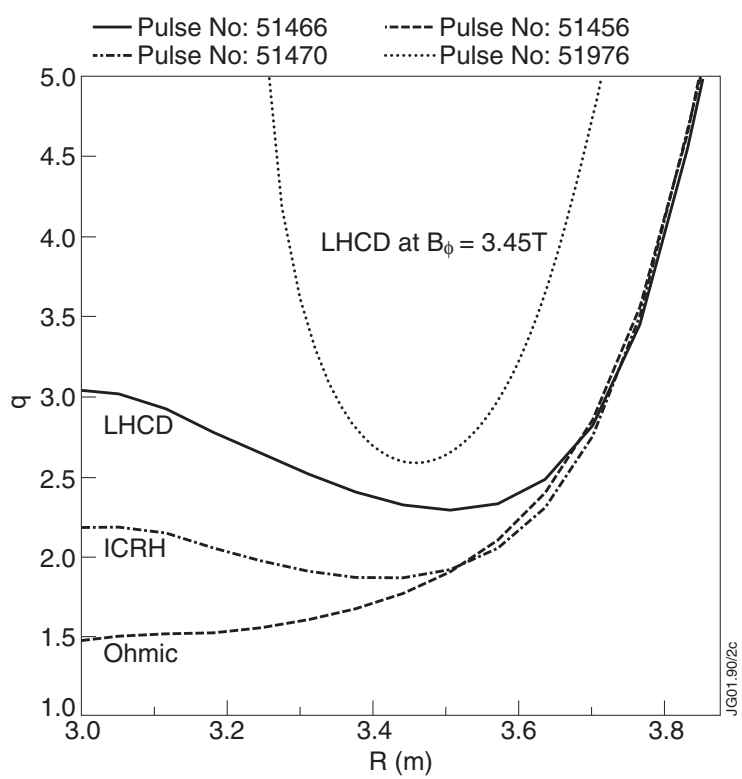

Figure 22. Overview of $q$ profiles showing the outer half of the plasma obtained in JET using different heating and current drive methods during the current ramp (from Tala et al (2002)). The heating and current drive systems available at JET are NBI, ICRH and LHCD. The $q$ profiles are inferred from equilibrium calculations constraint by MSE measurements. The most efficient method to generate reversed magnetic shear is the electron heating and off-axis current drive by lower hybrid waves (LHCD). Keeping current, its ramp rate, density and lower hybrid power the same, stronger magnetic shear reversal is achieved at larger toroidal magnetic field $\left(B_{\phi}=3.45 \mathrm{~T}\right)$. Off-axis ICRH, mainly heating the electrons, produces weak reversed shear, while in the absence of auxiliary heating (ohmic) the $q$ profile remains monotonic. 
et al 1994, Takase et al 1996, Baylor et al 1997). Additional ion cyclotron resonance heating, which is applied just after the injection of the pellet, distinguishes the PEP mode from earlier results of confinement improvement by pellet injection in Ohmically heated plasmas (Greenwald et al 1984, Wolfe et al 1986). The PEP mode has been identified with negative magnetic shear $s=(r / q)(\mathrm{d} q / \mathrm{d} r)<0$ and $q_{0}>1$ in the plasma centre (Hugon et al 1992, Baylor et al 1997), fulfilling the requirement for transport reductions and high- $n$ ballooning stability. Both, the shear reversal and $q_{0}>1$ are caused by the perturbation of the pellet, which by depositing large amounts of cold particles in the plasma centre produces flat or even hollow temperature profiles. Connected to the electron temperature by the plasma conductivity, a current profile of similar shape is formed. The off-axis bootstrap current generated by the strong pressure gradients, following the core confinement improvement, further supports the favourable modifications of the magnetic shear and the $q$ profile, extending the duration of the high performance phase. However, due to the lack of current and pressure profile control MHD instabilities limit the duration of the PEP mode to a few energy confinement times. The formation of an internal transport barrier in the PEP mode is evidenced not only by the increase of the density gradient, but, in particular, by a reduction of the temperature gradient length, after the plasma helped by ICRH has recovered from the initial cooling by the pellet (Hugon et al 1992, Takase et al 1996). For the ions, this indicates the stabilization of ITG modes (Baylor et al 1997).

In contrast to pellets, which cool the plasma without a loss of energy by exchanging density against temperature, radiation cooling by high- $Z$ impurities can have the same effect on the current density profile (Koslowski et al 1997), but at the expense of an energy loss, which is not compensated by possible confinement improvements. In this context, the effect of pellet injection on temperature, current profile and confinement bears more resemblance to the formation of hollow electron temperature profiles and hence hollow conductivity profiles by off-axis ECRH in RTP (de Baar et al 1997).

Another early example of an internal transport barrier was produced in the DIII-D tokamak. Studying the effect of a reduction of the internal inductance on plasma stability, the creation of a slightly hollow current profile by neutral beam heating during an increase of the plasma current is followed by a strong central pressure profile peaking (Lazarus et al 1991).

As pointed out by Lackner et al (2000), internal transport barriers can be classified according to the ratio of ion to electron heating. In a burning fusion plasma the dominant heat source will be the fast $\alpha$-particles mainly heating the electrons. The fusion reaction rate will require high density, which means that collisional energy exchange between electron and ions will also be high. With the exception of a few examples like the PEP mode, this is not the case in plasmas with ITBs in present day experiments, which at low densities exhibit only a weak coupling of the two plasma components. However, concerning the ratio of ion to electron temperatures, the full range from $T_{\mathrm{i}}>T_{\mathrm{e}}, T_{\mathrm{i}} \approx T_{\mathrm{e}}$ and $T_{\mathrm{i}}<T_{\mathrm{e}}$ has been covered in these experiments. In the following, the discussion of the results from the various tokamak devices is categorized accordingly.

\subsection{Internal transport barriers with $T_{\mathrm{i}}>T_{\mathrm{e}}$}

In present day tokamaks, one of the dominant heating methods is the injection of neutral particles, which for the purpose of heating the plasma must have energies clearly above the thermal energy of the plasma. With the conventional technique of extracting positive ions from a plasma source, energies up to $\approx 150 \mathrm{keV}$ can be reached. This, however, restricts the penetration depth of the beam, in particular, at the high plasma densities $\left(\approx 1 \times 10^{20} \mathrm{~m}^{-3}\right)$ envisaged in a burning fusion plasma. Therefore, recent developments aim for higher energies up to $1 \mathrm{MeV}$ which requires negative ion sources in order to achieve sufficiently 
high neutralization rates for the conversion of the ion into a neutral beam (Okumura et al 2000, Vollmer et al 2000). For heating purposes normally hydrogen or deuterium are used, while in special applications also tritium or helium have been injected.

The heating of a minority population of ions (normally hydrogen) by the resonant absorption of ion cyclotron resonance frequency (ICRF) waves produces ion energies in the $\mathrm{MeV}$ range, which is equivalent to ion velocities of the order of the thermal electron velocity. Hence, in the thermalization process of the fast ions a large fraction of the power goes into the electrons, depending on the minority concentration and the amount of direct coupling of the ICRF waves to the main plasma ion population (e.g. deuterium) at a higher harmonic. It should be noted that at the high ion temperatures reached in ITBs of larger tokamaks, this direct damping process turns ICRH from heating mainly the electrons into a heat source with a substantial fraction of ion heating.

In contrast, neutral beam injection (NBI) with its much lower ion energies predominantly heats the thermal plasma ions. In the absence of strong collisional coupling at low plasma densities $\left(<5 \times 10^{19} \mathrm{~m}^{-3}\right)$ this leads to $T_{\mathrm{i}}>T_{\mathrm{e}}$, which is the case in the majority of neutral beam heated plasmas with ITBs. Internal transport barriers assisted by neutral beam heating have been attained in many tokamaks (Koide et al 1994, Levinton et al 1995, Strait et al 1995, JET Team (presented by C Gormezano) 1997, Wolf et al 1999a). They all feature low or negative magnetic shear in the core plasma region. The neutral beam injection is not only the dominant heating source, but also plays a decisive role in the suppression of the turbulence in providing toroidal momentum, which, through generating toroidal plasma rotation, contributes to the $E \times B$ shearing rate. Another possible effect is the central fuelling of the plasma by NBI which, by increasing the density peaking, can stabilize ITG mode turbulence.

3.1.1. JET. In the JET tokamak two types of internal transport barriers are distinguished, the optimized shear (OS) and the reversed shear (RS) regimes. First, the optimized shear regime was developed (JET Team (presented by C Gormezano) 1997, JET Team (presented by F X Söldner) 1997, Sips et al 1998, Cottrell et al 1998, Baranov et al 1999, JET Team (presented by F X Söldner) 1999, Gormezano 1999, Becoulet et al 2000, Challis et al 2001a). With moderate auxiliary heating during the current ramp, using mainly ICRH, $q$ profiles with low or weakly negative magnetic shear and $q>1$ are formed. Subsequently, the ITB is established by a combination of ICRH and NBI. Figure 23 shows a comparison between an OS and a RS ITB plasma.

The first successful ITBs with the fusion reactor relevant fuel mixture of about equal amounts of deuterium and tritium were achieved in this OS regime (Gormezano et al 1998). They exceeded the performance of the steady state ELMy H-mode in terms of fusion power ( $\leqslant 8.2 \mathrm{MW})$, triple product $n_{\mathrm{i}}(0) T_{\mathrm{i}}(0) \tau_{\mathrm{E}} \leqslant 1.1 \times 10^{21} \mathrm{~m}^{-3} \mathrm{keV} \mathrm{s}$ and power multiplication factor $Q_{\mathrm{DT}} \approx 0.4$ (Söldner et al 1999, JET Team (prepared by C Gormezano) 1999), but still falling short of the transiently reached performance of the ELM-free H-mode discharges (Keilhacker et al 1999). Although the sustainment of the ITB was complicated by the lower $\mathrm{H}$-mode threshold with tritium, the reduction in electron and ion transport seems similar in DD and DT plasmas (JET Team (prepared by C Gormezano) 1999). In fact, one of the main obstacles of extending the ITBs in JET and and also in other tokamaks to power levels clearly above the H-mode threshold is the incompatibility with the large Type-I ELMs, which, although an instability of the edge H-mode transport barrier, seem to perturb the core plasma also and thus destroy the ITB (JET Team (presented by F X Söldner) 1997, Sarazin et al 2000).

In H-mode, quasi-stationary conditions are achieved partly by ELM activity (Zohm 1996, Hubbard 2000) limiting the edge confinement to a level, which is compatible with the requirements for a stationary distribution of energy, particles and impurities inside the 

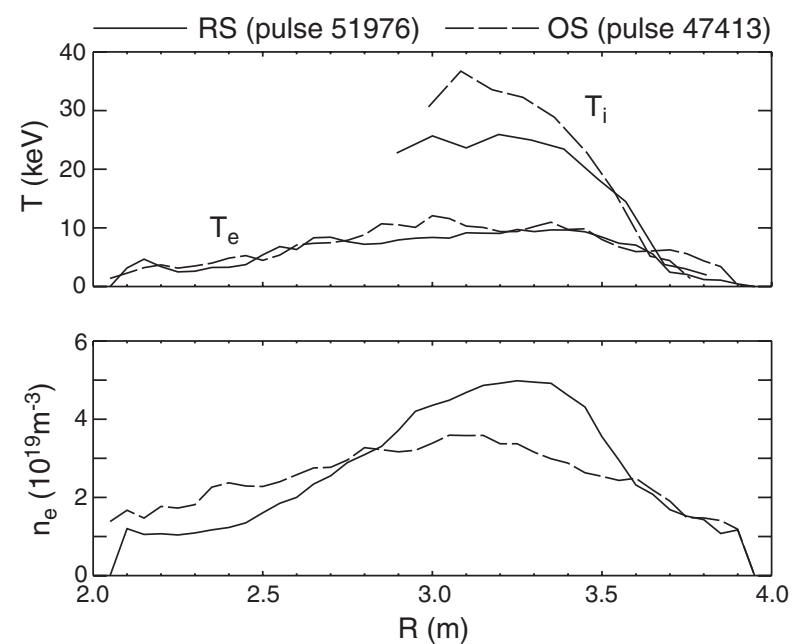

Figure 23. Examples of internal transport barriers in JET. Compared are the profiles of electron temperature, $T_{\mathrm{e}}$, and density, $n_{\mathrm{e}}$, and of the ion temperature, $T_{\mathrm{i}}$. While the neutron rates are the same in both cases, the profiles of temperature and density are different. In the OS case, the central $T_{\mathrm{i}}$ is higher, but the density profile is less peaked. The ion temperature gradients in the ITB region are comparable. The electron temperature profile peaking is weak in both cases. The heating powers for producing these profiles differ considerably. In the OS case (3.5 MA plasma current and 3.45 T toroidal magnetic field) $29 \mathrm{MW}$ (19 MW NBI and $10 \mathrm{MW} \mathrm{ICRH)} \mathrm{is} \mathrm{required,} \mathrm{while} \mathrm{in}$ the RS case (2.5 MA plasma current and 3.45 T toroidal magnetic field) $21 \mathrm{MW}$ (17 MW NBI and $4 \mathrm{MW}$ ICRH) is sufficient.

plasma. The ELM stability is thought to be determined by the interplay between ballooning stability, driven by the edge pressure gradient, and the peeling mode stability, driven by the edge current density and improved by the edge pressure gradient (Connor et al 1998, Wilson and Miller 1999). ELMs cause relaxation oscillations, i.e. the collapse of the edge pressure gradient, which follows the destabilization of the ELM, produces a stable situation, resulting in a renewed build-up of the pressure.

There are various categories of ELMs. Type-I ELMs exhibit the lowest repetition rate. Accordingly, a single Type-I ELM expels large fractions of the plasma energy. Extrapolating this to a fusion reactor, Type-I ELMs may lead to intolerable heat fluxes reaching the target plates. Type-III ELMs are usually obtained at heating power levels not too far above the $\mathrm{H}$-mode threshold. Their occurrence is higher in frequency and hence the perturbations of edge and core plasma is limited. Other forms of edge MHD activity which exhibit a favourable more continuous heat exhaust are Type-II ELMs (Stober et al 2001a and references therein), quasi-coherent modes (Hubbard et al 2001) and edge harmonic oscillations (Greenfield et al 2001). In DIII-D the latter facilitates the combination of internal and edge transport barriers, with an edge pressure gradient otherwise only seen in conjunction with Type-I ELMs (see section 3.1.4).

In JET, ITBs have been combined with L- and $\mathrm{H}$-mode edge conditions. The latter include edge transport barriers with both Type-III and Type-I ELMs and have been termed double transport barriers or double barrier (DB) modes (Söldner et al 1999, JET Team (presented by F X Söldner) 1999). However, long sustainment of ITBs to date was only possible with the smaller Type-III ELMs, but at the expense of a lower pedestal pressure. In discharges with L-mode or Type-III ELMy edge the problem is how to avoid Type-I ELMs, as the applied 
heating power levels usually lie above the power threshold for the transition to Type-I ELMs ${ }^{12}$. One possible method to suppress strong ELM activity, which has been applied in JET OS discharges, is the injection of argon or other noble gases into the plasma periphery (Gormezano 1999). Possible explanations of this ELM mitigation include the cooling of the edge plasma inside the last closed flux surface by impurity radiation and thus a reduction of the edge pedestal pressure below the instability threshold of Type-I ELMs (Becoulet et al 2000). However, argon tends to enter the core of the plasma diluting the fuel ions. In addition, the analysis of the carbon charge exchange recombination spectra, used to derive the ion temperature, is partially compromised by the presence of argon lines in these spectra (Zastrow et al 1999).

ITBs with reversed magnetic shear are obtained in JET by either applying off-axis ICRH or having a prolonged phase of off-axis LHCD (Challis et al 2001a) in the current ramp. The latter technique facilitates a large range of negative magnetic shear values (see figure 22). As the lower hybrid waves not only drive off-axis current but also heat the electrons, electron ITBs with the central $T_{\mathrm{e}}$ in excess of $10 \mathrm{keV}$ can form (Ekedahl et al 1998, Hogeweij et al 2002). Figure 23 compares the two types of JET ITB regimes. The examples are chosen such that, for the temperature and density profiles shown, the neutron rates and also the energy content of the plasmas do not differ by more than $10 \%$. Nevertheless, the profiles of temperature and density are different. In the OS case the central $T_{\mathrm{i}}$ is higher, while the density profile is less peaked. The ion temperature gradients in the ITB regions are comparable. Also, the electron temperature profile peaking is weak in both plasmas, partly due to the limited electron heating. The heating power required for producing the temperature and density values in the OS plasma is higher by $40 \%$, which is also reflected in the lower $\mathrm{H}$-factor of $H_{\text {ITER } 89-\mathrm{P}}=2.3$, as compared to $H_{\text {ITER89-P }}=3.3$ for the RS case. In conjunction with the strong density peaking, however, high- $Z$ impurities tend to accumulate more strongly in the centre of the RS ITBs (Dux et al 2001) (see also section 4.2).

The lower power threshold for ITB formation is another distinctive feature of RS discharges (Becoulet et al 2001a) (see section 4). Also, the dependence of this threshold on the magnetic field is not evident in RS cases. Connected to this are higher neutron rates at a given power level or, in other words, the same neutron rate at lower power (Challis et al 2002) (figure 24). Nevertheless, the overall highest neutron yields have been obtained in the OS plasmas due to improved stability, mainly caused by the lower pressure profile peaking at higher heating powers (Huysmans et al 1999) (see section 4.3).

The better confinement of reversed shear discharges, on the other hand, is reflected in larger bootstrap current fractions but also in the tendency to disrupt ${ }^{13}$. At reduced $\beta$, by limiting the heating power, and with decreased plasma current the favourable alignment of pressure and current profiles for high confinement and bootstrap current fractions has been exploited to develop ITBs, which approach steady state conditions (Crisanti et al 2002, Litaudon et al 2002). At $90 \%$ of non-inductive current drive with a $50 \%$ contribution from the bootstrap current, ITBs have been sustained for $27 \tau_{\mathrm{E}}$ or about one resistive time scale for current diffusion (skin time). This is facilitated also by the avoidance of strong ELM activity. Similar to the effect of ramping up the total plasma current, this is possibly caused by the increase of the edge current density by continuous LHCD during the main heating phase, lowering the edge stability threshold and hence initiating ELM activity at a lower pedestal pressure (Becoulet et al 2001b, Becoulet et al

\footnotetext{
12 In JET, the ability to form ITBs and keep the plasma edge in L-mode depends on the divertor configuration. While with the 'Mark-IIa' divertor ITBs with L-mode edge plasmas were obtained regularly, this was not possible anymore with the more closed 'Mark-IIGB' ('GB' for gas box) divertor, where the edge, on increasing the heating power, immediately went into $\mathrm{H}$-mode. The reason for this different behaviour is unknown.

${ }^{13}$ In a tokamak the complete loss of confinement due to a sudden drop in the plasma current is called disruption. The occurrence of disruptions leads to considerable mechanical forces and heat loads.
} 


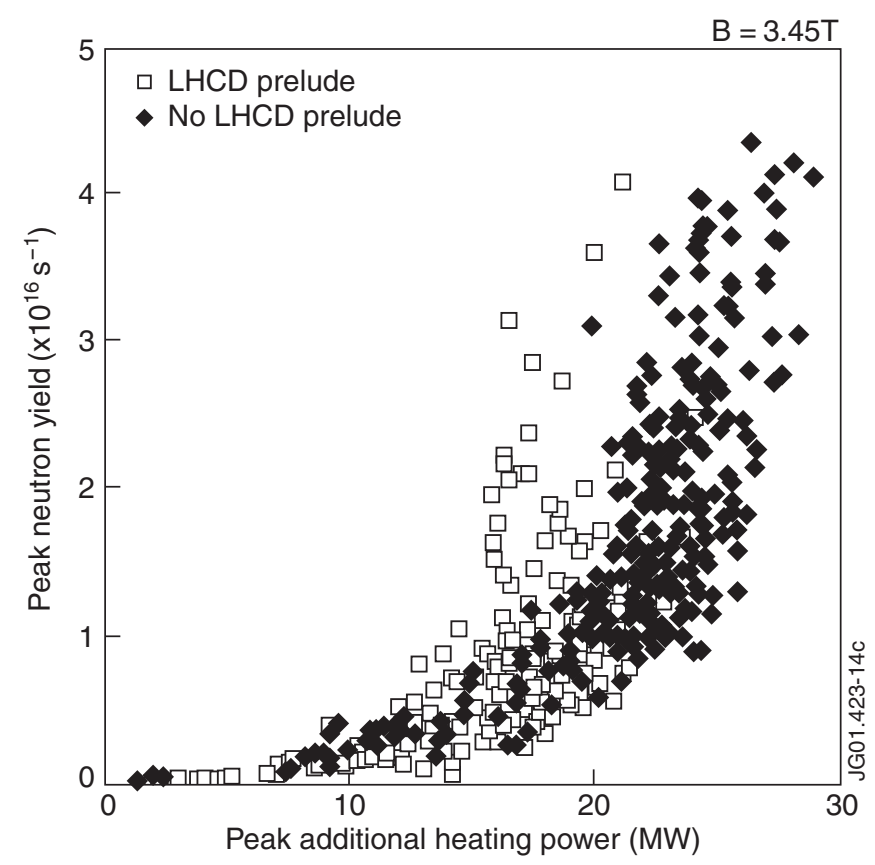

Figure 24. Dependence of the peak neutron rate plotted on the additional heating power for the two types of JET ITB regimes (from Challis et al (2002)). The RS ITBs have been produced at a toroidal magnetic field of $B_{\phi}=3.45 \mathrm{~T}$ by LHCD during the current ramp, in contrast to the OS ITBs, which have been generated at the same magnetic field but without LHCD. Although higher performance is achieved at lower power levels in the RS regime, the overall highest performance is obtained in the OS regime.

2002). With the help of a real time feedback control scheme, not only keeping the neutron rate at a prescribed value, but also controlling the gradient length at the transport barrier, the feasibility of full non-inductive current drive, lasting several seconds, has been demonstrated at reduced plasma current in JET reversed shear plasmas (Crisanti et al 2002, Mazon et al 2002).

3.1.2. JT-60U. Internal transport barriers have been obtained in JT-60U for different types of $q$ profiles, ranging from weakly positive to negative magnetic shear (Koide et al 1994, Fujita et al 1997a, b, Koide et al 1998b, Kamada et al 1999a, Ide et al 2000). Similar to JET, there is a distinction between basically two confinement modes in JT-60U ITB discharges. The RS modes correspond to each other in both tokamaks, while with respect to the $q$ profile shape the high- $\beta_{\mathrm{p}}$ mode resembles the JET OS regime. However, as the name of the confinement mode already suggests, $\beta_{\mathrm{p}}$ and bootstrap current fractions are higher in the JT-60U high- $\beta_{\mathrm{p}}$ plasmas. In contrast to JET, NBI is not only used as the main heating method during the ITB phase but also for tailoring the current profile during the current ramp. In JET this is not effective (Tala et al 2002). Reasons are the limitation of the heating power to avoid undue heat fluxes onto the wall components opposite to the beam injectors and the limited electron heating efficiency. The first is a consequence of low power absorption at low plasma density. The second is aggravated by the narrow central power deposition at low density, which leads to insufficient slowing down of the current diffusion.

Another major difference between JT-60U and JET, but also the tokamaks DIII-D and ASDEX Upgrade, is the spatial configuration of the heating beams (Koide et al 1997). While 
in the latter devices the toroidal orientation is the same for all beams, in JT-60U a balance between the power injected parallel (co) and opposite (counter) to the toroidal plasma current is possible (see section 4.1). As a consequence of the different torques transferred to the plasma by NBI, the turbulence stabilizing terms in the $E \times B$ shearing rate and their effect on transport can differ significantly (Koide et al 1998b, Shirai et al 1998, 1999, 2000). With ideally balanced NBI the toroidal rotation in the plasma should be zero. Different fast ion orbits or orbit losses, depending on the injection direction of the fast ions, result in deviations from this (see figure 28). Another effect to be considered is the higher toroidal magnetic field ripple in JT60-U compared to JET (van Blokland et al 1994). The increased fast ions losses, associated with the field ripple, on the one hand reduce the local momentum transfer and on the other hand produce radial currents, both effects influencing the toroidal rotation profile.

In addition to the medium energy beams, JT-60U is the first tokamak equipped with a negative ion source beam injection (N-NBI) system, delivering heating powers of $\approx 5 \mathrm{MW}$ at neutral beam energies of $360 \mathrm{keV}$ (Okumura et al 2000). The increase of the beam energy leads to a considerable shift of partition of the heat fluxes towards electron heating.

The two different types of ITB regimes in JT-60U are shown in figure 25. As in JET, ITBs have been observed with positive and negative magnetic shear in the centre of the plasma. The positive shear regime is termed high- $\beta_{\mathrm{p}}$ mode (Koide et al 1994, Fujita et al 1997a, Koide et al $1998 \mathrm{~b}$, Kamada et al 1999a), owing to high values of $\beta_{\mathrm{p}}$ at relatively low plasma current and high toroidal magnetic field. This results in large fractions of non-inductively driven current, as the bootstrap current fraction scales with $\beta_{\mathrm{p}}$. The high- $\beta_{\mathrm{p}}$ mode in JT-60U is distinguished by low density and high central ion temperatures, facilitated by strong central heating and fuelling by NBI into sawtooth free discharges. The ITB is mainly seen in the density and ion temperature profiles, which exhibit breaks in their gradients (figure 25(b)), while the electron temperature profile does not always show such changes. Going from positive to weakly negative magnetic shear the ion temperature profiles develop a box-like shape with a plateau in the centre (Koide et al 1997). It is worth noting, that this is not necessarily a feature of an ITB. As pointed out in section 2.3.2, the ion confinement degradation in the centre of many ITBs may be to some extent associated with increased neoclassical transport in the presence of large central $q$ values.

Similar to JET, in JT-60U the reversed shear regime (Fujita et al 1997a) develops steeper gradients of density and temperature in particular of $T_{\mathrm{e}}$ (figure 25(a)). As illustrated in figure 13 (section 2.3.1), this is associated with very localized drops of the electron and ion heat conductivities. The improvement of the electron transport with negative magnetic shear may be important in the view of a burning fusion plasma which relies on strongly coupled ion and electron transport and electron heating by $\alpha$-particles. In JT-60U the highest performance, although not in steady state, has been achieved in reversed shear ITB plasmas. Assuming deuterium and tritium as plasma fuel, the extrapolated fusion power of such discharges corresponds to $Q_{\mathrm{DT}}^{\mathrm{eq}}$ of up to 1.25 (Ishida et al 1997, Fujita et al 1999).

Comparing the high- $\beta_{\mathrm{p}}$ and the RS discharges (Koide et al 1998b, Kamada et al 1999a), the RS plasmas tend to have better performance, which is however difficult to sustain for longer periods of time. One of the problems is that the $q$ profile is still evolving, which results in the termination of the high performance phase, driven by the large pressure gradients in the low shear region, when $q_{\text {min }}$ reaches 2 (Kamada et al 1999a) (see section 4.3). Generally, the high- $\beta_{\mathrm{p}}$ discharges exhibit better steady state properties, however, at lower performance. This is illustrated in figure 26 , which compares the achieved duration of $\beta_{\mathrm{N}} \times H_{\text {ITER89-P }}$ and $Q_{\mathrm{DT}}^{\mathrm{eq}}$ for different types of discharges. At a low plasma current of $1 \mathrm{MA}$ full non-inductive current drive with a $75 \%$ bootstrap current fraction has been reached in the high- $\beta_{\mathrm{p}}$ regimes (not shown in figure 26). 

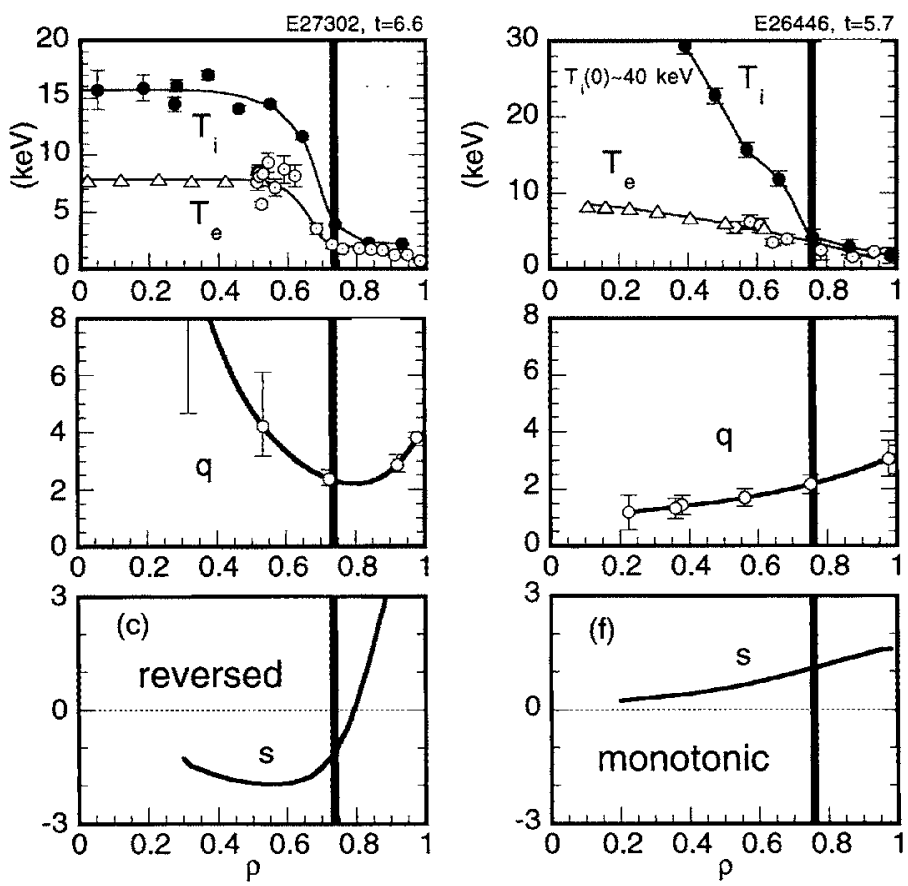

Figure 25. Comparison of the JT-60U ITBs with $(a)$ negative magnetic shear in the plasma centre and $(b)$ throughout positive magnetic shear (reproduced from Koide et al (1998b)). The latter is termed high $\beta_{\mathrm{p}}$ mode. Shown are the profiles of temperature, $T_{\mathrm{e}}$ and $T_{\mathrm{i}}$, safety factor, $q$, and magnetic shear, $s$. In the high $\beta_{\mathrm{p}}$ discharges ITBs are associated mainly with changes of the density and ion temperature gradients. In the presence of negative magnetic shear also strong reductions of the electron thermal heat conduction are observed. With RS the transport barrier lies in the vicinity of the minimum of the $q$ profile.

Similar to JET, the reversed shear discharges also could be prolonged with the help of feedback control of the plasma pressure, keeping it low enough to avoid any immediate $\beta$-collapse (RS $2.4 \mathrm{MA}, q_{95}=3.4$ in figure 26 ). A further reduction of the plasma current and pressure permits such ITBs to be sustained quasi-stationary. Thus, at $\beta_{N} \approx 2$ and $H_{\text {ITER89-P }} \approx 3.5$ full non-inductive current drive with a bootstrap current fraction of $80 \%$ has been sustained for about $6 \tau_{\mathrm{E}}$ (Fujita et al 2001b). Also here, as in JET, it is important that the bootstrap current from the ITB is well aligned with the current profile supporting high confinement. Since the discharge duration of $6 \tau_{\mathrm{E}}$ (equivalent to $2.7 \mathrm{~s}$ ) is below the resistive time scale for current diffusion, the stationarity of the current profile is concluded from the radially constant loop voltage profile, which is derived from MSE measurements (Forest et al 1994).

In contrast to JET, there appears to be no problem in JT-60U in combining ITBs with H-mode edge barriers and Type-I ELMs even for longer periods of time (Kamada et al 1999a, b). The reason for this different behaviour is unclear. Some of the JT-60U plasmas are operated at low plasma current corresponding to high $q_{95}$, where ELMs are less perturbative. In line with the ballooning stability limit the critical pressure gradient, at which Type-I ELMs are destabilized, decreases approximately like $I_{\mathrm{p}}^{-2}$ (Suttrop et al 1997). Hence, lower current means a less violent collapse of the edge pressure when the ELM occurs. For plasmas with high $q_{95}$ and high triangularity even the complete disappearance of strong ELMs has been observed. Calculations of high- $n$ ballooning stability suggest that this change of the ELM activity is caused by opening the access to the second stability regime at the plasma edge (Kamada et al 

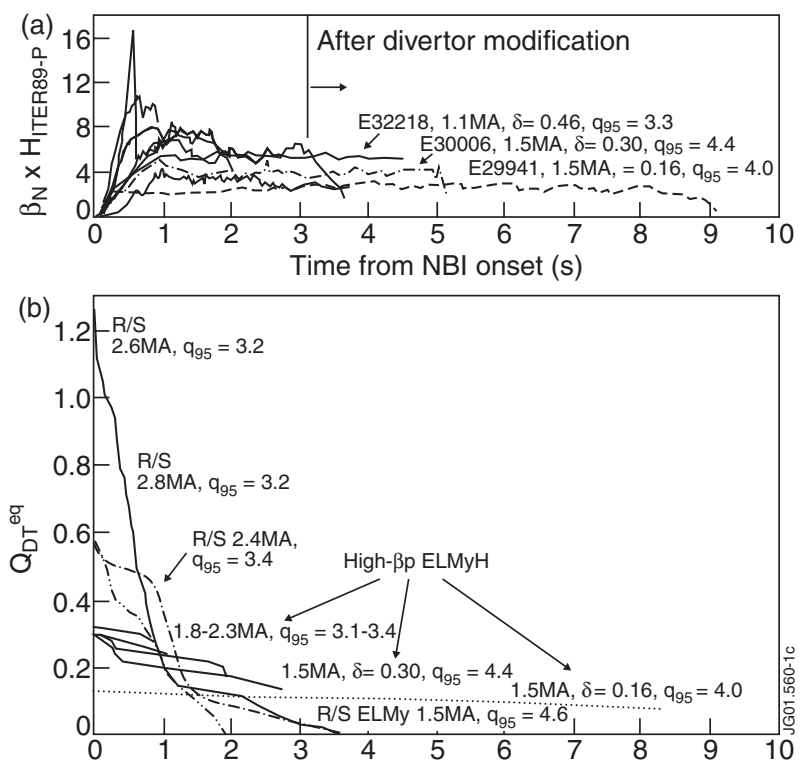

Figure 26. Comparison of JT-60U high- $\beta_{\mathrm{p}}$ and RS discharges with respect to sustainment of $(a)$ $\beta_{N} \times H_{\text {ITER } 89-\mathrm{P}}$ and $(b) Q_{\mathrm{DT}}^{\mathrm{eq}}$ (reproduced from Kamada et al (1999a)). Note that in $(a)$ the timescale starts at the onset of the NBI and in $(b)$ reflects the duration of the high performance phase. Generally, as in other tokamaks, sustainment of confinement and stability is only possible at reduced performance. Higher triangularity, $\delta$, raises the performance. As indicated in $(a)$, the possible discharge durations have been extended by the introduction of a pumped divertor configuration (Kamada et al 1999b). In particular at high heating power the control of the particle content of the plasma has been improved.

2000). It should be noted, that the larger toroidal magnetic field ripple compared to JET may influence the behaviour of the plasma edge transport and stability (van Blokland et al 1994).

3.1.3. TFTR. Of the tokamaks discussed in this section, TFTR is the only one with a circular cross section and a limiter configuration. In contrast to the X-point configuration of divertor tokamaks, in a limiter tokamak the confined plasma inside the last closed flux surface is in direct contact with the first wall components. This proximity to the wall prevents the build-up of the edge pressure gradients characteristic for the H-mode. In fact, limiter configurations have been utilized also in divertor tokamaks to suppress the H-mode transition (Rice et al 1996a, Wolf et al 2000). Thus, the potential difficulty of combining ITBs with ELMs does not apply to TFTR. Similar to JT-60U, the neutral beam injectors of TFTR point in opposite toroidal directions, resulting in the possibility of balanced NBI.

In TFTR ITBs are produced and sustained by NBI heating (Levinton et al 1995, Synakowski et al 1997a, b Bell et al 1998b,1999a). Starting with moderate NBI power in the current ramp, to preform the reversed shear profile, the power is increased up to $30 \mathrm{MW}$. In some of these RS discharges a sudden transition into an improved confinement regime was observed, referred to as enhanced reversed shear (ERS) mode. As shown in figure 27 this transition manifests itself essentially as an increase of the core density, while ion and electron temperatures (with $T_{\mathrm{i}}>T_{\mathrm{e}}$ ) are comparable in the RS and ERS regimes (Levinton et al 1995). The equal $q$ profiles (within error margins) at the ERS transition suggest a bifurcation process, 

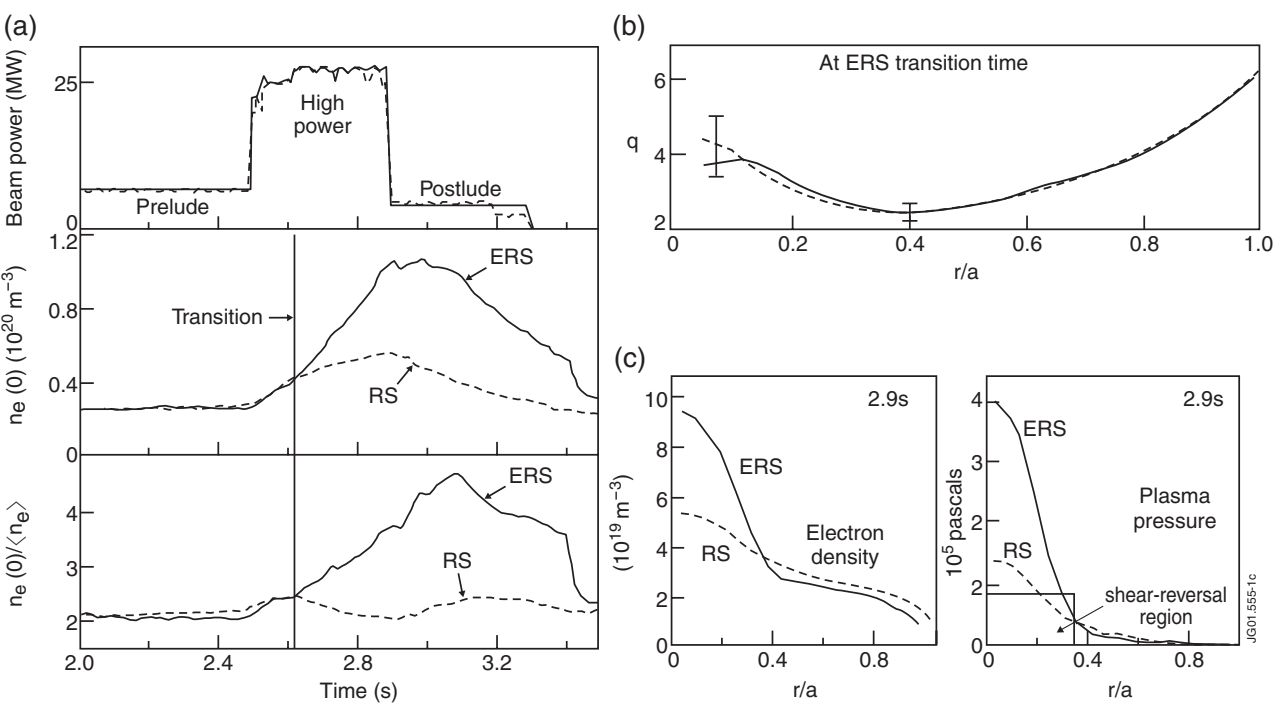

Figure 27. Comparison of RS and enhanced reversed shear (ERS) mode in TFTR (reproduced from Synakowski et al (1997b)). (a) Time evolution of NBI heating power, central density, $n_{\mathrm{e}}(0)$, and density peaking factor, $n_{\mathrm{e}}(0) /\left\langle n_{\mathrm{e}}\right\rangle$. The reversed magnetic shear is formed during the current ramp in the prelude phase. High power is required to trigger the ERS transition. The power may be reduced in the postlude phase, to study, for instance, the back transition to lower confinement. (b) The $q$ profiles at the ERS transition are identical within the error margins. (c) The ERS mode manifests itself essentially as an increase of the core electron density, resulting in a strong increase of the electron pressure.

which may be triggered by minute differences in pressure or current profiles. In many cases the ERS transition is accompanied by spatially and temporarily confined excursions of the poloidal plasma rotation, leading to very localized peaks of the radial electric field of up to $200 \mathrm{kV} \mathrm{m}^{-1}$. The origin of this $E_{\mathrm{r}}$ behaviour is understood only rudimentarily. However, it has been confirmed independently by measurements of the poloidal plasma rotation (Bell et al $1998 \mathrm{a}$ ) and $E_{\mathrm{r}}$ directly (Levinton et al 1998) (see section 5.3). Possible theoretical explanations include the formation of jets of $E \times B$ flow (Staebler 2000) and $E_{\mathrm{r}}$ bursts, produced by radial fast particle losses due to MHD instabilities (Günter et al 2001a). The latter is used to explain a similar observation in ASDEX Upgrade (De Pena-Hempel et al 1998) (see section 4.1).

Besides the Type-I ERS mode described above, which has a power threshold of about $20 \mathrm{MW}$ in the main heating phase, a different ERS transition has been observed at heating powers as low as $5 \mathrm{MW}$ (Bell et al 1996, 1998b). This Type-II ERS regime also exhibits reductions of ion, electron and particle transport. The latter, however, is not as strong as in the Type-I ERS mode. The Type-II transition is characterized by a sudden increase of $T_{\mathrm{i}}$ and $T_{\mathrm{e}}$ and only a gradual rise of $n_{\mathrm{e}}$.

The proximity of $q_{\min }$ to 2 at the time of the transition to the Type-II ERS regime suggests the involvement of rational $q$-surfaces as a possible trigger mechanism (Bell et al 1996). In many other tokamaks there are clear indications that the appearance of rational $q$ values in an evolving current profile can trigger ITBs (Koide et al 1994, Greenfield et al 1999, Joffrin 2001, 2002a, Güenter et al 2001a) (see section 4.1).

For completeness it should be mentioned that also in TFTR a few ITBs with a fuel mixture of deuterium and tritium have been attempted (Bell et al 1997). As it was found that the already high power threshold for accessing the ERS regime (Type-I) had increased further with the 
addition of tritium, the discharges tended to approach the $\beta$-limit soon after the confinement transition. These and other difficulties resulted only in fusion power levels distinctly below the expectations from DD operation. Considering that the JET ITB experiments with DT were more successful, but without clear results with respect to the effect of tritium on confinement, this aspect of the extrapolation of ITBs to a burning fusion plasma still remains unclear.

3.1.4. DIII-D. In the DIII-D tokamak reversed magnetic shear plasmas have been investigated under a wide range of conditions (Strait et al 1995, Rice et al 1996a,b,c, Forest et al 1996, Lao et al 1996, Greenfield et al 1997, 1999, Rice et al 1999a, Doyle et al 2000). This negative central shear (NCS) regime, which in figure 28 is compared to a JT-60U reversed shear plasma, has many common aspects with the other ITB modes discussed in this section. Similar to JT-60U, TFTR and ASDEX Upgrade (see below), neutral beam heating during the current ramp is used to tailor the current profile (Rice et al 1996c). As in JET and ASDEX Upgrade, the main heating source is uni-directional NBI, strongly driving toroidal plasma rotation (Strait et al 1995, Rice et al 1996a, b). Simulations of the plasma turbulence suggest
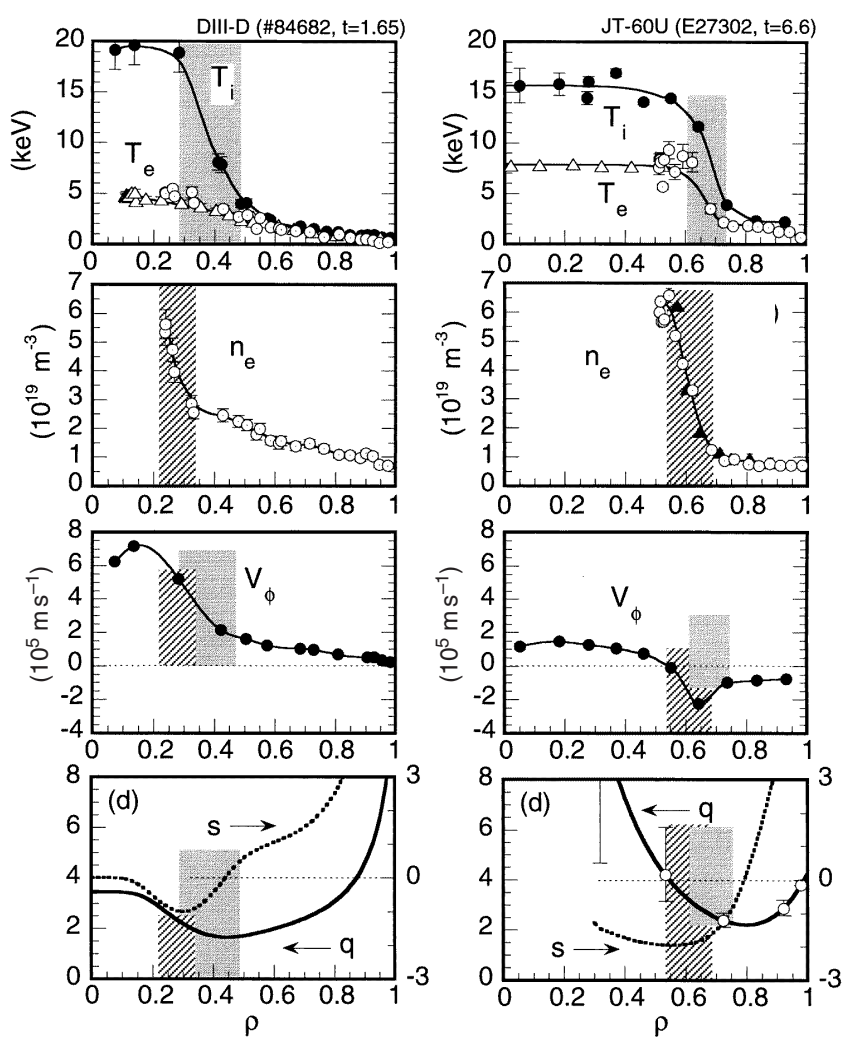

Figure 28. Comparison of DIII-D and JT-60U ITBs with negative magnetic shear, $s=$ $(r / q)(\mathrm{d} q / \mathrm{d} r)$, in the plasma centre (from Koide et al (1998a)). Common features are strong gradients of ion temperature, $T_{\mathrm{i}}$ (shaded areas), and electron density, $n_{\mathrm{e}}$ (hatched areas). The stronger electron temperature, $T_{\mathrm{e}}$, gradient in JT-60U may be associated with more negative magnetic shear in the ITB region. Although the toroidal rotation due to the different beam configurations is distinctively different in both devices, the local gradients of $v_{\phi}$, in JT-60U observed as a notch at the location of the ITB, contribute significantly to the $E \times B$ shearing rate in DIII-D (Lao et al 1996a) and JT-60U (Shirai et al 2000). 
that the shear of $v_{\phi}$ contributes significantly to the formation of the ITB both in DIII-D (Lao et al 1996a) and JET (Budny et al 2002). In agreement with the other tokamaks, negative (or reversed) magnetic shear generally facilitates reductions of the electron thermal transport, the ion thermal and momentum transport, and the particle transport (Doyle et al 2000).

The DIII-D ITBs have been observed also in conjunction with weakly negative (Lao et al 1996a, Greenfield et al 1997) or positive magnetic shear (Greenfield et al 1999). Consistent with the observations in other tokamaks at least for the plasma ions, negative magnetic shear is not necessary to obtain or sustain an ITB. Generally the ITB power threshold is rather low in DIII-D. For negative magnetic shear and with NBI in co-current direction it lies below $2.5 \mathrm{MW}$ (Greenfield et al 2000a). For positive magnetic shear the necessary $4 \mathrm{MW}$ still seems low. However, in relative terms the corresponding heat flux is not so different to the power threshold of 10-20 MW of the JET positive (optimized) shear cases, considering the larger size of the latter device.

The high flexibility of DIII-D in producing fast changes of the plasma configuration is utilized to control the power threshold of the H-mode transition by changing the bias between the lower and upper X-point (Greenfield et al 1997). As the H-mode threshold is higher, if the ion $\nabla B$ drift points away from the X-point, and lower, if it points towards the $\mathrm{X}$ point, L- to H-transitions can be induced by changing the vertical plasma position. With this technique the influence of the edge confinement properties on the confinement and stability of internal transport barrier plasmas has been investigated (Lazarus et al 1996, Rice et al 1996a, Strait et al 1997). It has been found that the broadening of the pressure profile, which is associated with the H-mode transition, makes it less sensitive to pressure driven MHD instabilities. Thus, if the $\mathrm{H}$-mode transition takes place before the pressure gradient at the ITB reaches critical values, $\mathrm{H}$-mode operation allows the discharge to achieve higher $\beta_{N}$ values.

In combination with strong plasma shaping (elongation $\kappa=2.15$ and triangularity $\delta=0.9$ ) this resulted in an impressive value of $Q_{\mathrm{DT}}^{\mathrm{eq}}=0.32$ for a device of this size (Lazarus et al 1996). However, DIII-D plasmas which combine ITBs with an H-mode edge are also susceptible to MHD instabilities. In ELM-free phases the large pressure gradients and the rise of the current density near the edge eventually become MHD unstable (Strait et al 1997). Here, both, edge bootstrap current and the continuous current ramp contribute to the edge current density. With ELMs, like in JET, the coupling of strong ELMs into the core plasma limits the duration of the central confinement improvement (Rice et al 1999a).

Injecting the neutral beam particles opposite to the plasma current (counter-NBI), for which the plasma current has to be reversed compared to standard operation, a sustainable combination of internal and edge transport barriers has been achieved in DIII-D (Burrell et al 2001, Doyle et al 2001, Greenfield et al 2001), resulting in stationary values of $\beta_{\mathrm{N}}=2.9$ and $H_{\text {ITER89-P }}=2.6$. Figure 29 compares the temperature, density and $q$ profiles of such a quiescent double barrier (QDB) plasma with those of an L-mode and an ITB with L-mode edge, the latter also with counter-NBI. The ion and electron temperature difference between QDB and ITB with L-mode edge is basically given by the additional pedestal temperatures. The main feature of the QDB regime is the quiescent $\mathrm{H}$-mode edge, which is combined with an ITB and is responsible for the name of the regime. Although the edge pressure gradients and the pedestal pressure resemble those of an H-mode with Type-I ELMs, the energy is released in a more continuous heat flow, which is in contrast to the short energy bursts characteristic for Type-I ELMs. This behaviour is similar to the quasi-coherent modes in Alcator C-Mod (Hubbard et al 2001). However, amongst other things, frequency range and edge collisionality suggest that the edge harmonic oscillations observed in the quiescent $\mathrm{H}$-mode edge are a different type of MHD modes (Doyle et al 2001). The QDB mode not only exhibits the more favourable absence of Type-I ELMs, from a divertor heat load point of view, but also avoids the perturbation of the 
(a)

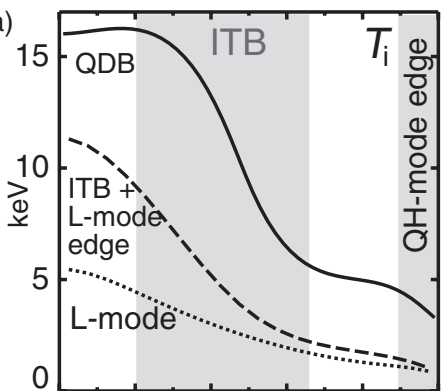

(c)

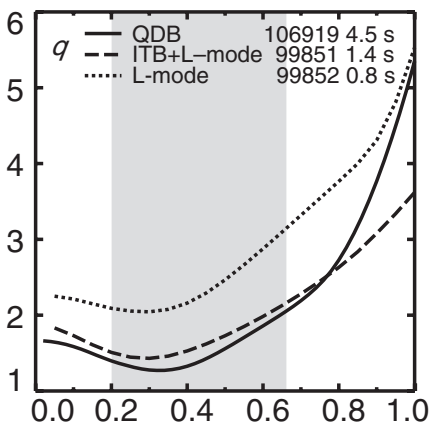

(b)
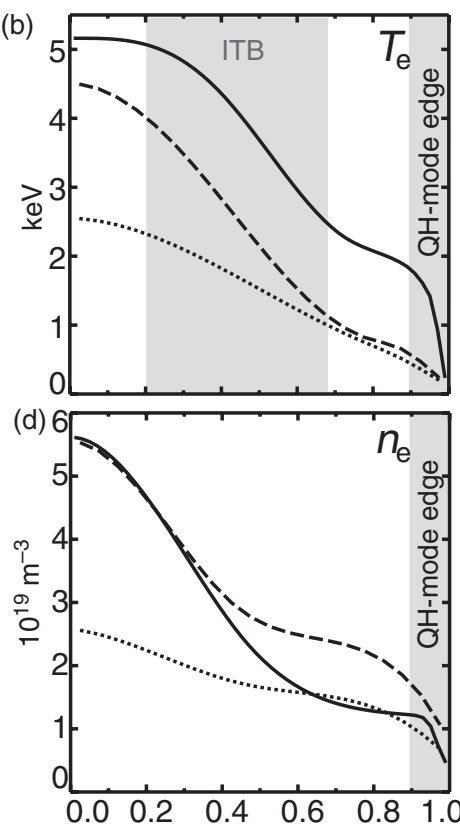

Figure 29. Profiles of (a) $T_{\mathrm{i}}$, (b) $T_{\mathrm{e}},(c) q$ and $(d) n_{\mathrm{e}}$ comparing an L-mode plasma (with reversed magnetic shear), an ITB with L-mode edge and the quiescent double barrier mode (QDB) (from Doyle et al (2001)). Shaded areas indicate regions of internal or edge transport barriers.

ITB by strong ELMs, which has been documented in DIII-D (Rice et al 1999a) and JET (JET Team (presented by F X Söldner) 1997, Sarazin et al 2000).

The effect of the counter-NBI is twofold. First, the compared to co-NBI modified edge ion orbits, may play a role in the different edge behaviour. Second, the stable separation of edge and internal transport barriers by a low confinement region is attributed to the modified contributions of rotation velocity and pressure gradient terms to the radial electric field, since counter-NBI is synonymous to a change of sign of the toroidal rotation term, $v_{\phi} B_{\theta}$, in the radial force balance equation. While for co-NBI the $E \times B$ shearing rate remains large over the whole plasma cross section, reversing the plasma current with respect to the angular momentum supplied by NBI results in an $\omega_{E \times B}$ which goes through zero between edge and core (Burrell et al 2001, Greenfield et al 2001).

However, similar to JET, there are indications of high- $Z$ impurity accumulation in the QDB mode which, as in JET, are associated with the strong density peaking (Doyle et al 2001) (see section 4.2).

3.1.5. ASDEX Upgrade. In the ASDEX Upgrade tokamak, which in size and shape is similar to DIII-D, a variety of $q$ profiles are generated by changing the time and level of NBI heating power during the current ramp. The $q$ profiles range from a monotonic shape with zero magnetic shear in the plasma core and $q_{0} \approx 1$ to negative central shear and $q_{\text {min }}>1$.

In the first case, fishbone oscillations, an instability driven by the fast neutral beam particles, clamp the current profile and hence prevent the appearance of the sawtooth instability (Günter et al 1999, Wolf et al 1999b). Thus, improved stability and confinement could be

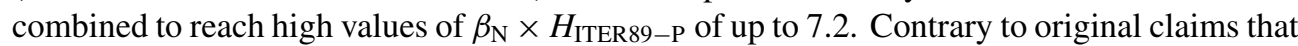
this also involved internal transport barriers (Gruber et al 1999b, Wolf et al 1999b), according 
to the ion temperature gradient length criterion (Peeters et al 2001a) discussed in section 2.3, these plasmas do not exhibit ITBs (Peeters et al 2001b) during their stationary phase. In the initial phase ITBs have been obtained transiently (Joffrin et al 2002b), which however decay to stiff temperature profiles as soon as the H-mode transition takes place. This example shows, that, obtaining an ion heat conductivity, which, within error bars of the power balance analysis is close to neoclassical transport levels in conjunction with an increase of the temperature gradient, may not be enough to decide whether an ITB is present. As long as the gradient length still does not deviate from other $\mathrm{L}$ - and $\mathrm{H}$-mode cases, residual turbulence must still be present, clamping the temperature profile at its critical gradient. To what extent the increased density profile peaking may be regarded as a different confinement state is not taken into account in the temperature gradient length criterion.

As described above for the other tokamaks, in ASDEX Upgrade too negative magnetic shear in the central plasma regions combined with NBI enables the formation of ITBs (Wolf et al 1999a, Güenter et al 2000b, Wolf et al 2000, 2001a). As illustrated in figure 30 the heating power, $q$ profile and ITB evolution are closely coupled. Both transport theory, which suggests a link between low or negative magnetic shear and the suppression of turbulence, and the bootstrap current at the ITB relate the evolution of the ITB to that of the $q$-profile (see section 4.1). Without external off-axis current drive the current diffusion can only be slowed down, which limits the sustainment of such ITBs to about $10 \tau_{\mathrm{E}}$.
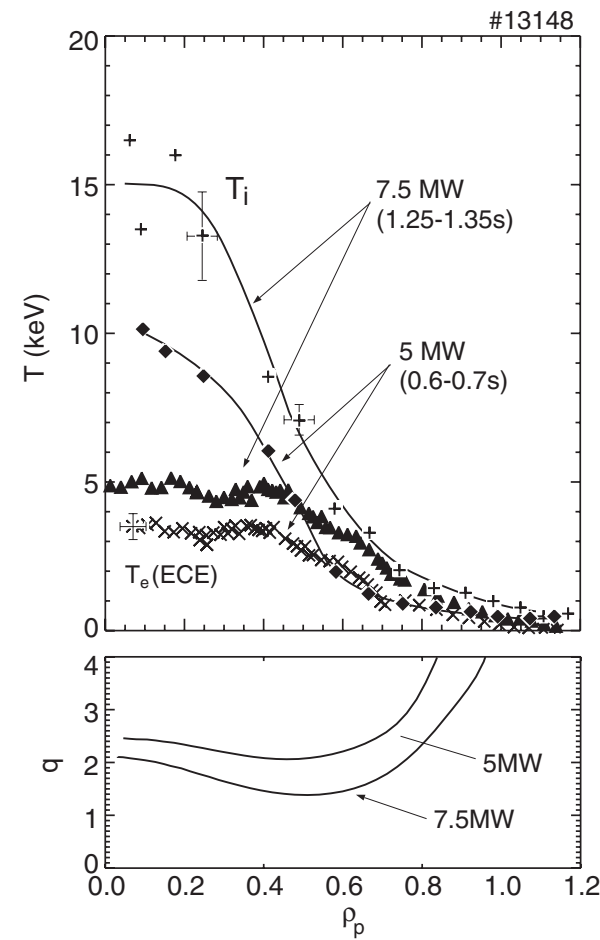

Figure 30. Evolution of an internal transport barrier in ASDEX Upgrade, generated by negative magnetic shear in the plasma centre (from Wolf et al (2001a)); copyright in these materials is vested in the International Atomic Energy Agency, Vienna, Austria from which permission for publication must be obtained). The regions of both large electron and ion temperature gradients move outwards, as the heating power is increased from 5 to $7.5 \mathrm{MW}$. Transport and bootstrap current link the minimum of the $q$ profile to the location of the ITB. 
In ASDEX Upgrade ITBs with high central pressure the transition of the plasma edge into $\mathrm{H}$-mode has to be avoided for stability reasons. This is facilitated by either the contact to the inner wall of the vessel or, comparable to DIII-D, by increasing the H-mode power threshold, changing from a lower to an upper X-point configuration and thus inverting the ion $\nabla B$ drift. Both, strong ELM activity and the destabilization of an external kink mode can terminate the ITB phase. The latter is caused by the additional edge bootstrap current of the H-mode pressure pedestal in combination with large core pressure gradients as soon as the edge $q$ becomes rational. Up to now the potentially beneficial effect of the $\mathrm{H}$-mode transition, improving the MHD stability due to a broadening of the pressure profile, has not been achieved in ASDEX Upgrade. The pressure, corresponding to the temperature profiles shown in figure 30 for the later time, is already close to the theoretical pressure limit without a conducting wall (Günter et al 2000a). At $\beta_{N} \approx 1.7$ this finally causes the disruptive termination of the discharge, when the H-mode transition occurs with $q_{95}$ in the vicinity of 4 .

An ITB at lower plasma current and higher heating power has been attained in ASDEX Upgrade in the high- $\beta_{\mathrm{p}}$ regime (Hobirk et al 2001, Wolf et al 2001a). Similar to the JT-60U plasmas these conditions are obtained by strong central heating (up to $10 \mathrm{MW}$ of NBI) into low density plasmas $\left(<3 \times 10^{-19} \mathrm{~m}^{-3}\right)$. This generates sawtooth free discharges with internal transport barriers and an H-mode edge. In contrast to JT-60U, the Greenwald density is reached during the high power phase. However, bearing in mind that the Greenwald density scales with the plasma current (Greenwald et al 1988), the absolute density values are nevertheless only moderately high. Owing to $\beta_{\mathrm{p}}$ values as high as 3.1, however, at the expense also of a high $q_{95}=9$, nearly full non-inductive current drive is accomplished by a combination of large bootstrap and neutral beam current fractions. In figure 31 the composition of such a current profile is shown. As illustrated by the $q$ profile derived from the current diffusion calculation, the magnetic shear reversal would be expected from the large bootstrap current, which by definition approaches zero at the plasma centre. The measurement, although in general agreement with the calculation, cannot resolve this feature. As discussed in section 3.1.2, the ELMs do not seem to adversely affect the ITB at low plasma current.

In summary, internal transport barriers with $T_{\mathrm{i}}>T_{\mathrm{e}}$ are characterized by an ion thermal transport close to neoclassical levels as far as it is possible to judge, considering experimental and theoretical inaccuracies. This appears to be valid for positive and negative magnetic shear. Negative magnetic shear, however, produces more localized and stronger confinement improvements of electron heat and particle transport, bearing in mind that the electron heat flux nevertheless stays above its neoclassical level. As discussed below, the good particle confinement can cause problems, in particular with the accumulation of high- $Z$ impurities (section 4.2). According to turbulence simulations negative magnetic shear is predicted to reduce the anomalous electron heat transport due to ETG and trapped electron modes. As ETG modes are thought to increase only the heat flux, the stabilization of the TEMs could be responsible for the improvement of the particle transport. For the reduction of the ITG driven heat conduction, magnetic shear and sheared $E \times B$ flows can be equally important, explaining why ion ITBs are observed for such a wide range of magnetic shear values. Since $E \times B$ shear flows are ineffective in suppressing ETG driven turbulence, electron ITBs in the presence of positive shear may be related to the stabilization of trapped electron modes.

\subsection{Internal transport barriers with $T_{\mathrm{i}}<T_{\mathrm{e}}$}

As discussed in the previous section, plasmas with $T_{\mathrm{i}}>T_{\mathrm{e}}$ are produced at densities low enough to decouple the two plasma components and with heating methods which predominantly supply energy to the ions, such as NBI and minority ICRH at high ion temperatures. Correspondingly, 

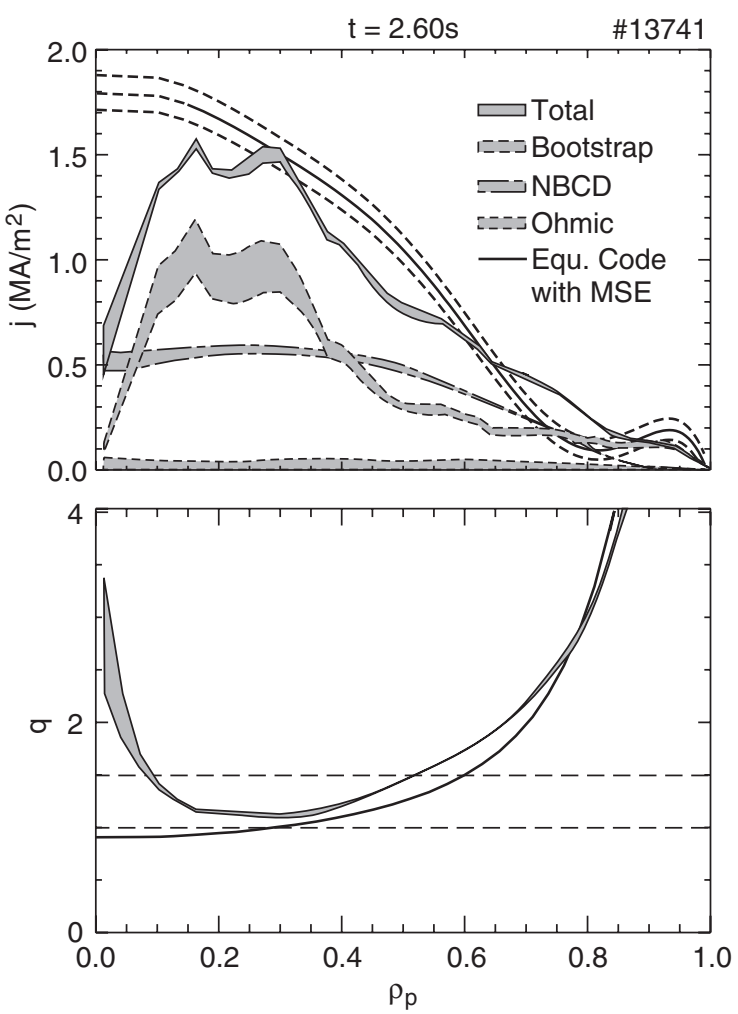

Figure 31. Comparison of calculated and measured current and $q$ profiles for a high $\beta_{\mathrm{p}}$ discharge in ASDEX Upgrade (reproduced from Wolf et al (2001a); copyright in these materials is vested in the International Atomic Energy Agency, Vienna, Austria from which permission for publication must be obtained). The composition of the current density profile is inferred from a current diffusion calculation taking the time history of the plasma current and neoclassical conductivity into account. Within the error bars the Ohmic current is zero. The fractions of neutral beam driven current (NBCD) and bootstrap current are $(44 \pm 2) \%$ and $(56 \pm 6) \%$, respectively. The bootstrap current distribution dominates the shape of the total current profile, which is in general agreement with the profile, derived from the equilibrium code and constrained by MSE measurements. The resolution of the measurement, however, does not allow the calculated negative magnetic shear in the plasma centre to be confirmed.

at low density electron heating schemes generate $T_{\mathrm{i}}<T_{\mathrm{e}}$. They include ECRH, ICRH at low minority concentrations and low ion temperatures, fast wave electron heating (FWEH), electron heating by lower hybrid waves and NBI at high energies using negative ion sources.

Although not relevant to burning fusion plasma with respect to the primary goal of achieving high fusion power, which needs high ion temperatures and densities, plasma regimes with $T_{\mathrm{i}}<T_{\mathrm{e}}$ can be exploited to study electron transport. Due to the strong collisional coupling of ions and electrons in a burning fusion plasma, ion and electron transport are equally important, as the confinement will be determined by the strongest transport channel. Extrapolating the results from the plasma conditions with $T_{\mathrm{i}}<T_{\mathrm{e}}$ to a fusion reactor type plasma, one should, however, remember, that $T_{\mathrm{i}} \approx T_{\mathrm{e}}$ may imply modified electron transport properties.

In this section the experimental evidence regarding the effect of magnetic shear on electron transport is presented. The RTP and TEXTOR results (Lopes Cardozo et al 1997, de Baar et al 
1997, Hogeweij et al 1998, de Baar et al 1999, Schüller et al 2000), which indicate a close link between the arrangement of rational $q$-surfaces and alternating layers of low and high confinement, suggesting a different transport mechanism (see section 2.3), will not be further addressed.

Besides the commonly used technique to modify the current profile by heating during the current build-up utilizing the skin effect, many electron heating methods also include the possibility of driving plasma current (Bernabei et al 1982, Leuterer et al 1985, Anado et al 1986, Saoutic et al 1997, Itoh et al 1999, Sauter et al 2000). As was demonstrated in Tore Supra with off-axis LHCD in the co-current direction (Litaudon et al 1996), and in TCV (Pietrzyk et al 2001), T-10 (The T-10 Team (presented by D A Kislov) 2001) and ASDEX Upgrade (Wolf et al 2001a) with central electron cyclotron current drive (ECCD) in the counter-current direction, negative central magnetic shear can be generated without the use of current ramps.

The main feature of these electron heating experiments is that in conjunction with negative magnetic shear an increase of the inverse electron temperature gradient length is observed (Barbato 2001) with respect to the value of $R / L_{T_{\mathrm{e}}} \approx 10$ in conventional confinement regimes with monotonic $q$ profiles (Ryter et al 2001b). The influence of the $q$ profile on the electron confinement is exemplified in figure 32. Two ASDEX Upgrade discharges with ECCD (Wolf et al 2001a) in the plasma centre are compared. Central heating and current drive in the direction of the plasma current supports the natural current profile shape with positive magnetic shear and $q_{0}<1$. In this case $R / L_{T_{\mathrm{e}}} \approx 11$ over an extended region of the plasma cross section. Driving the central current in the opposite direction, negative magnetic shear with $q>1$ develops and $R / L_{T_{\mathrm{e}}}$ increases by almost a factor of two to $\approx 21$ in the negative shear region. Although some of this increase may be attributed to the absence of sawtooth oscillations, this can certainly not explain a factor of two. This kind of behaviour results in exceptionally high electron temperatures even in the smaller tokamaks FTU (Buratti et al 1999, Alladio et al 1999, Bracco et al 2001) and TCV (Pietrzyk et al 2001, Sauter et al 2001). The high central heat fluxes from ECRH in combination with the improved confinement leads to electron temperatures in excess of $10 \mathrm{keV}$ (Bracco et al 2001, Pietrzyk et al 2001). The corresponding thermal electron heat conductivities lie at $1 \mathrm{~m}^{2} \mathrm{~s}^{-1}$ or below. Despite the much larger heat fluxes, these values are comparable to the transport in an Ohmic plasma, which is heated by the plasma current alone. Moving the ECRH deposition sufficiently offaxis, the electron temperature profiles tend to become hollow (Alladio et al 1999, Buratti et al 1999, Prater et al 2001) as the central electron heat flux is reduced and the cold ions constitute an energy sink for the much hotter electrons (figure 34). This effect should not be confused with the flat electron temperature profiles observed in ITB plasmas with high ion temperatures. There, in the absence of a net energy loss, as $T_{\mathrm{i}}>T_{\mathrm{e}}$, and without gradients driving ETG modes, the cause is not understood (see section 2.3.2).

In Tore Supra the electron confinement has been investigated using various other heating and current drive methods, including LHCD, FWEH (Equipe Tore Supra (presented by X Litaudon) 1996, Voitsekhovitch et al 1997, Hoang et al 1998) and minority ICRH (Hoang et al 2000). As illustrated in figure 33 for Tore Supra plasmas with lower hybrid heating, the electron confinement improves continuously as the shear is decreased (Equipe Tore Supra (presented by X Litaudon) 1996). This link between magnetic shear and a reduction of the electron transport has been confirmed by JET results (Hogeweij et al 2002). Off-axis LHCD, which in JET is normally used to establish reversed magnetic shear (figure 22), produces internal transport barriers of the electrons, which are located inside the minimum of the $q$ profile in the negative magnetic shear region. Independent of any changes of ITB position and $q$ profile, which can be a shrinking of the ITB by as much as $30 \mathrm{~cm}$, the ITB remains located at about the same value of negative magnetic shear. It should be noted that the dependence 
(a)
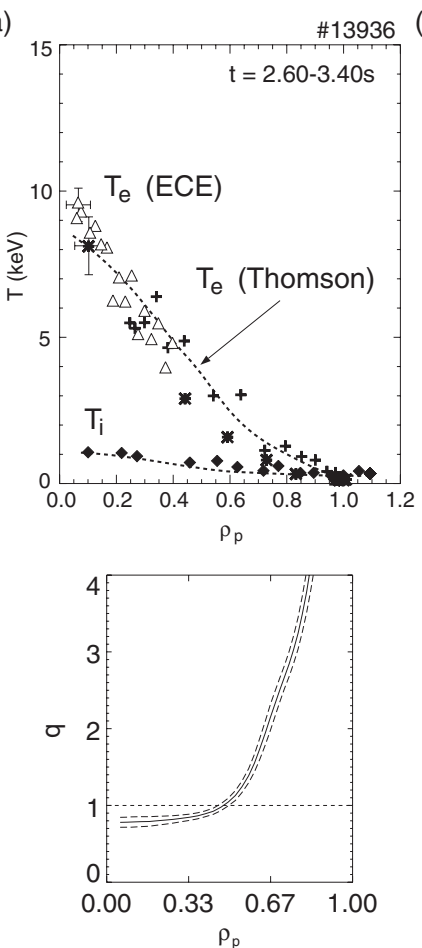

(b)
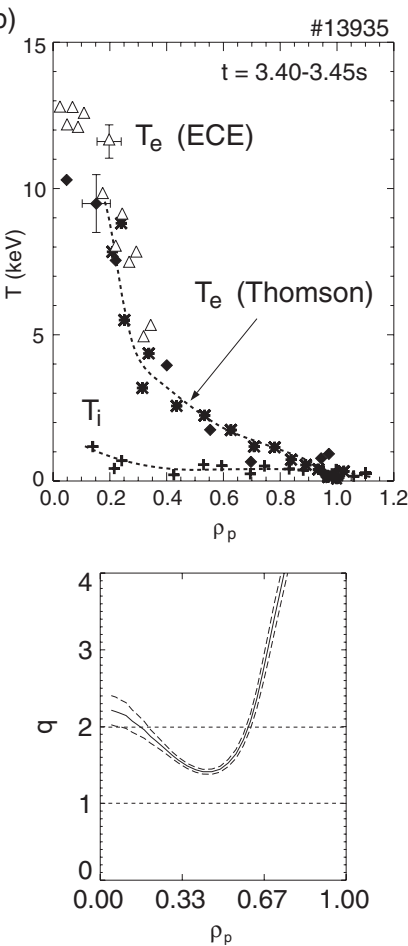

Figure 32. Comparison of temperature $\left(T_{\mathrm{e}}\right.$ and $\left.T_{\mathrm{i}}\right)$ and $q$ profiles for two ASDEX Upgrade discharges (reproduced from Wolf et al (2001a)); copyright in these materials is vested in the International Atomic Energy Agency, Vienna, Austria from which permission for publication must be obtained). (a) Central co-ECCD sustains the monotonic $q$ profile and $R / L_{T_{\mathrm{e}}} \approx 11$ remains low. (b) Counter-ECCD produces negative central magnetic shear and an electron ITB with $R / L_{T_{\mathrm{e}}} \approx 21$. Electron temperatures are measured by electron cyclotron emission (ECE) and Thomson scattering. The ion temperatures, inferred from CXRS, are an order of magnitude below $T_{\mathrm{e}}$. This is consistent with only $0.1 \mathrm{MW}$ of ion heating power compared to $1.5 \mathrm{MW}$ going to the electrons. The $q$ profiles are calculated by an equilibrium code using MSE measurements.

of the transport on the magnetic shear, shown in figure 33, does not cover the high magnetic shear (or high $l_{\mathrm{i}}$ ), where the heat conductivity is expected to decrease again.

In addition to the effect of the magnetic shear, $\alpha$-stabilization has been suggested as a mechanism to reduce the electron transport. Simulations of the linear ETG mode stability indicate that, in order to recover the measured electron temperature profiles of electron cyclotron heated DIII-D plasmas (Prater et al 2001), $\alpha$-stabilization caused by the additional Shafranov shift of the increased electron pressure is required (Greenfield et al 2000b, Doyle et al 2002) (figure 34). Although the ion temperature is low $(<1 \mathrm{keV})$, the ion transport in the core is approximately neoclassical, and also the heat flux delivered to the ions is low.

The stabilizing influence of low magnetic shear and increased plasma pressure is summarized in figure 35, showing the results of theoretical simulations of the non-linear ETG mode driven turbulence, which explains enhanced electron transport by the formation of radially elongated streamers (Jenko et al 2000). As the pressure, i.e. $\alpha$, increases, the magnetic shear can be increased, still retaining the low electron transport. At least qualitatively, this trade-off between high pressure or low magnetic shear is also reflected in the theoretical modelling of the experimental results. In DIII-D, on the one hand, $\alpha$-stabilization is invoked 


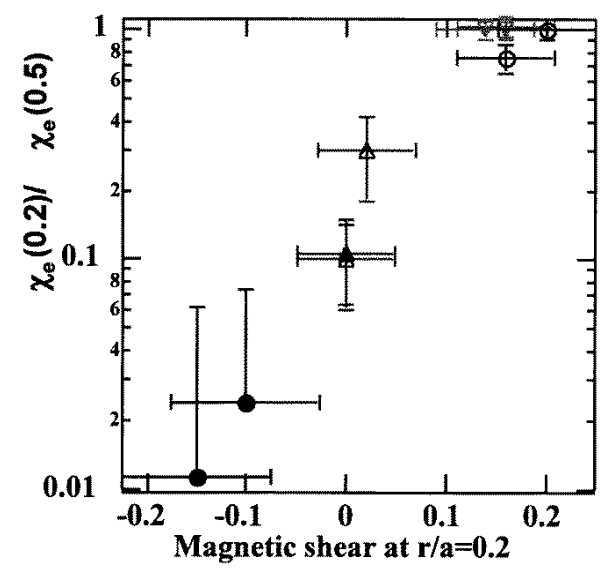

Figure 33. Dependence of the electron thermal transport on the magnetic shear for various Tore Supra plasmas with lower hybrid heating and current drive (from Equipe Tore Supra presented by $\mathrm{X}$ Litaudon 1996). The core electron heat conductivity at $r / a=0.2$ normalized to its half-radius value is shown. The magnetic shear is inferred from Faraday rotation angles (Soltwisch 1992). Obviously, the electron confinement improves by reducing the magnetic shear.

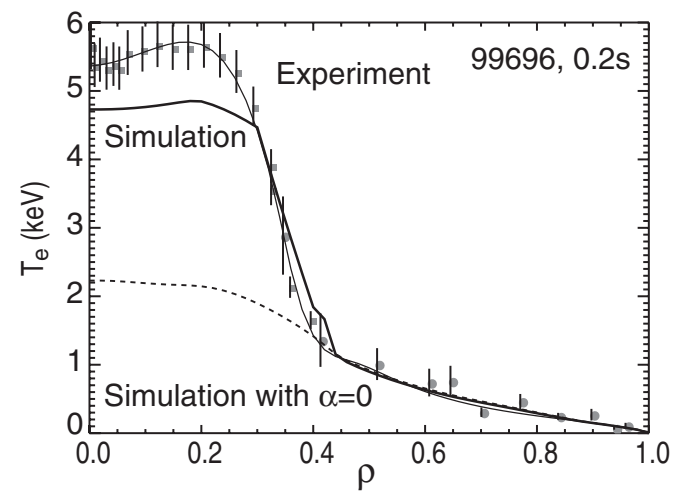

Figure 34. Comparison of experimental and simulated electron temperature profiles for a DIIID plasma with off-axis ECRH $(\rho \approx 0.3)$ during the ramp-up of the plasma current (reproduced from Doyle et al (2002); copyright in these materials is vested in the International Atomic Energy Agency, Vienna, Austria from which permission for publication must be obtained). In the absence of direct ion heating $T_{\mathrm{i}}<1 \mathrm{keV} \ll T_{\mathrm{e}}$. The electron ITB is only recovered by the theoretical simulations of the linear ETG mode stability, if $\alpha$-stabilization, caused by the additional Shafranov shift of the increased electron pressure, is invoked (Greenfield et al 2000b, Doyle et al 2002). Without $\alpha$-stabilization $(\alpha=0)$ the predicted $\nabla T_{\mathrm{e}}$ is substantially lower than the experimental one.

to explain the low electron transport. On the other hand, for instance in the Tore Supra plasmas discussed above, the electron pressure is too low to have any considerable effect. There, the transport reduction has to be caused mainly by magnetic shear stabilization.

\subsection{Internal transport barriers with $T_{\mathrm{i}} \approx T_{\mathrm{e}}$}

In a burning fusion plasma both the higher density and the heating by the $\mathrm{MeV} \alpha$-particles implies $T_{\mathrm{i}} \approx T_{\mathrm{e}}$. Increasing the plasma density at a given triangularity is known to degrade the 


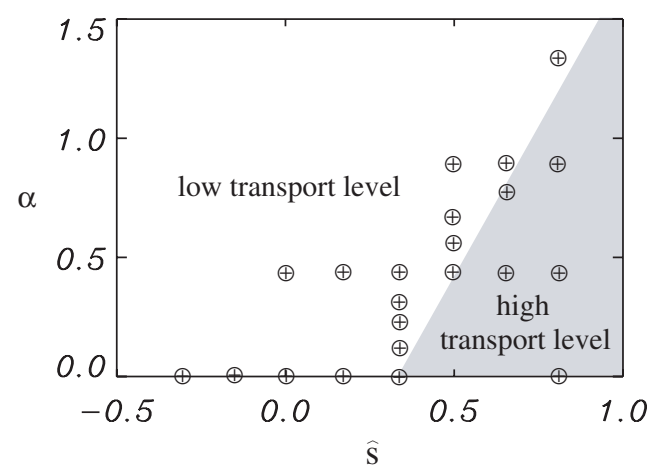

Figure 35. Qualitative behaviour of electron transport from non-linear ETG mode stability simulations (from Jenko et al (2000)). Low magnetic shear, $\hat{s}$, and high normalized pressure gradient, $\alpha=-q^{2} R_{0} \nabla \beta$ (ballooning parameter; $R_{0}$ is the major radius), span the region of low transport. The $\oplus$ 's denote the $(\alpha, \hat{s})$ pairs of the numerical simulations.

energy confinement of H-mode plasmas (Saibene et al 1999, Stober et al 2000). According to transport theory, making $T_{\mathrm{i}}$ and $T_{\mathrm{e}}$ equal may increase either the ion (Romanelli 1989, Garbet et al 1992, Kotschenreuther et al 1995) or the electron energy transport (Jenko et al 2001), depending on whether $T_{\mathrm{i}}$ or $T_{\mathrm{e}}$ were higher at the starting position. As most high performance plasmas with internal transport barriers feature $T_{\mathrm{i}}>T_{\mathrm{e}}$, this is a critical issue for their adaptability to reactor conditions.

The experimentally observed trend towards a confinement degradation as $T_{\mathrm{e}} / T_{\mathrm{i}}$ increases is exemplified in figure 36 for a number of JT-60U discharges (Kamada et al 1999a). The standard $\mathrm{H}$-mode at low triangularity shows the typical reduction of the $\mathrm{H}$-factor at higher $T_{\mathrm{e}} / T_{\mathrm{i}}$, which is associated also with an increase of the density. In the high- $\beta_{\mathrm{p}}$ regime the improvement obtained by raising the triangularity is similar to the observations in standard H-mode plasmas. Because of the considerable reduction of the electron heat transport, the reversed shear plasmas achieve the highest $\mathrm{H}$-factor for given $T_{\mathrm{e}} / T_{\mathrm{i}}$. Regarding the generalization of the trends shown in figure 36, caution is advised, since the change of $T_{\mathrm{e}} / T_{\mathrm{i}}$ may also involve the change of other plasma parameters influencing transport, such as toroidal momentum or energy content. In addition, it is not clear that the heating power available always allowed to reach the possible confinement or stability limits.

The experimental evidence regarding the effect of additional electron heating in plasmas with ITBs seems contradictory. Both examples with and without confinement degradation due to electron heating can be found.

Central counter-current drive, provided by either FWEH and current drive in DIII-D (Forest et al 1996) or by ECRH and current drive in ASDEX Upgrade (Wolf et al 2000, Günter et al $2000 \mathrm{~b}$ ) to enhance the negative magnetic shear in neutral beam heated discharges with already established ITBs, does not have any detrimental effect on the plasma confinement. In these cases the momentum transfer and the ion heating change little, as the electron heating is added together with the NBI. The temporal evolution and radial profiles of the discharges are shown in figures 37 and 38. In DIII-D the electron temperature rises sharply when the electron heating is switched on. The ion temperature continues to rise steadily until a transition to even higher electron and ion confinement is observed (figure 37(a)), coinciding with increases of temperature, density and $E \times B$ shearing rate (figure $37(b)$ ). This transition is attributed to the turbulence stabilizing effect of the increase of $\omega_{E \times B}$, caused by a reduction of the poloidal magnetic field in the plasma center due to the additional bootstrap current of the increased electron pressure (Forest et al 1996). 


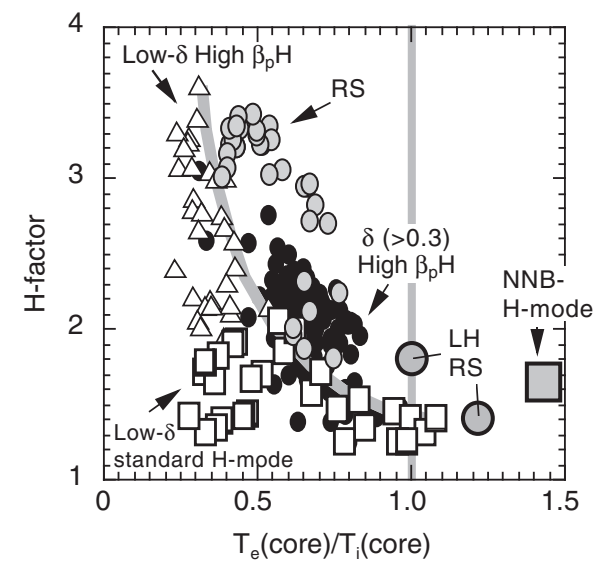

Figure 36. Dependence of $\mathrm{H}$-factor (L-mode scaling) on the temperature ratio $T_{\mathrm{e}} / T_{\mathrm{i}}$ in the plasma core $(r / a=0-0.2)$ for various types of JT-60U plasmas (from Kamada et al (1999a)). Standard H-mode plasmas at low triangularity, $\delta$, high- $\beta_{\mathrm{p}}$ H-mode plasmas with ITBs at low and high $\delta$, RS plasmas with ITBs, plasmas sustained by LHCD and H-mode plasmas with negative ion beam source NBI (NNB) are shown.
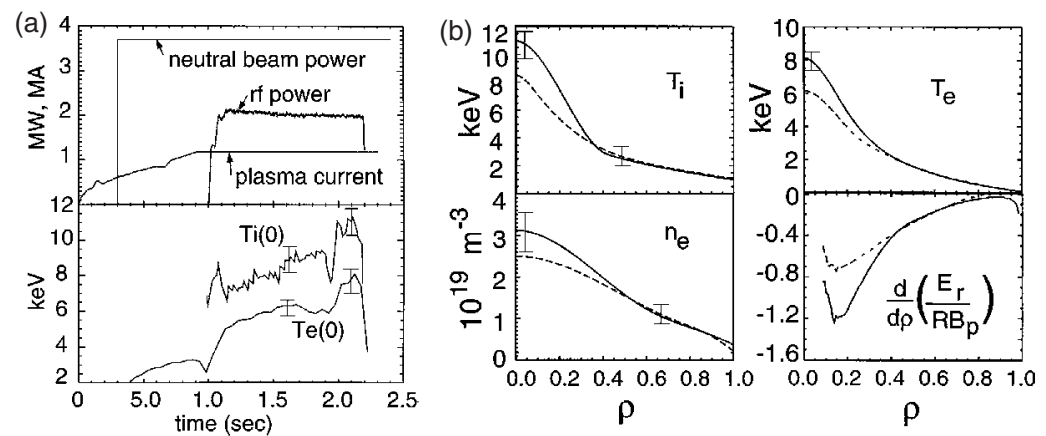

Figure 37. (a) Temporal evolution of plasma current, FWEH power (RF power), NBI power and central electron and ion temperatures for a DIII-D reversed magnetic shear discharge (from Forest et al (1996)). After the electron heating is switched on, ion and electron temperature continue to rise steadily until a transition to even higher confinement is observed. (b) The profiles of temperature, density and $E \times B$ shearing rate before (- - - ) and after the transition (- - ) show a clear increase of all four quantities. The lowering of the central poloidal magnetic field $\left(B_{\mathrm{p}} \equiv B_{\theta}\right)$ and the corresponding increase of $\omega_{E \times B} \propto(\partial / \partial r)\left|E_{r} /\left(R B_{\theta}\right)\right|$ (see equation (15)) are made responsible for the confinement improvement.

The phenomenology in ASDEX Upgrade is similar. Figure 38 compares the electron heated discharge with the reference case with NBI only. In these reversed shear plasmas $\left(q_{0}>q_{\mathrm{min}}\right)$ the rise of $T_{\mathrm{e}} / T_{\mathrm{i}}$ does not have any detrimental effect on the confinement. Despite the fivefold increase of the electron heat flux in the centre, neither the ion nor the electron heat conductivity increases. In fact, as a consequence of the higher electron temperature and hence lower collisional transfer from the beam ions to the electrons, the ion heat flux from NBI rises, resulting in an even higher ion temperature with additional electron heating (Wolf et al 2000). The explanation of the poloidal magnetic field reduction and its effect on $\omega_{E \times B}$ for the confinement improvement in DIII-D is also conceivable for the ASDEX Upgrade case. However, the turbulence stabilizing effect of the additional Shafranov shift ( $\alpha$-stabilization), 

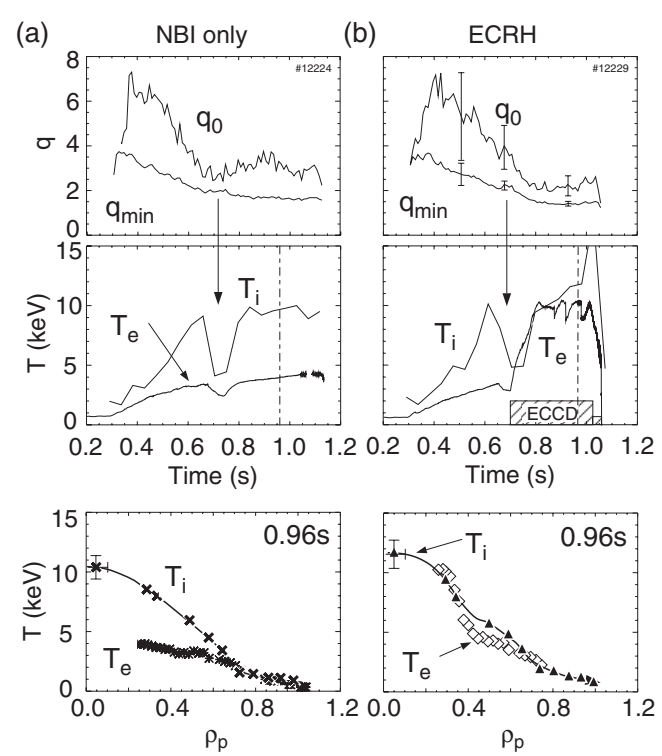

Figure 38. Comparison of (b) a discharge with combined NBI and central counter-ECCD with (a) a reference case with NBI only (from Wolf et al (2001a); copyright in these materials is vested in the International Atomic Energy Agency, Vienna, Austria from which permission for publication must be obtained). Displayed are the temporal evolutions of central and minimum safety factor, $q_{0}$ and $q_{\min }$, respectively, central ion and electron temperatures and the temperature profiles at a time when the ITBs are fully established. In these reversed shear plasmas $\left(q_{0}>q_{\min }\right)$ the rise of $T_{\mathrm{e}} / T_{\mathrm{i}}$ does not have any detrimental effect on the confinement. The transient loss of confinement at $0.7 \mathrm{~s}$ is caused by the destabilization of a double tearing mode when $q_{\min }$ reaches 2 (Wolf et al 2000) (see section 4.3.2).

owing to the rise of the electron pressure, seems to be sufficient to compensate the increased destabilization of the ITG modes at higher $T_{\mathrm{e}} / T_{\mathrm{i}}$ (Peeters et al 2001a). Regarding the portability of these results to a fusion reactor plasma, it should be noted that $T_{\mathrm{e}} \approx T_{\mathrm{i}}$ has been achieved by a similar heating of electrons and ions and, due to the low density, not by collisional heat transfer.

In contrast to the results reported so far, in ITB discharges obtained in DIII-D with low or slightly negative central magnetic shear the addition of pure ECRH or FWEH without current drive leads to a confinement degradation in both the ion and electron channel (Staebler et al 1998, Greenfield et al 1999). As illustrated in figure 39 the onset of the electron heating, here in the form of ECRH, is accompanied by a drop in the central ion temperatures and rotation velocities. The power balance analysis shows that, in addition to this considerable loss of ion and momentum confinement, the electron heat conduction also rises. In line with the general observation that even in ITB plasmas the electron transport remains anomalous (above its neoclassical level), calculations of the critical $R / L_{T_{\mathrm{e}}}$ for ETG mode instability in the presence of negative magnetic shear suggests that, even with an ITB, the electron temperature remains critical gradient limited (Stallard et al 1999). Thus, an increase of the heat flux would result in a rise in the electron heat transport. On the other hand, as discussed for the ASDEX Upgrade case and in section 3.2, the stabilizing influence of the additional electron pressure also has to be considered. Similarly, the response of the ion transport to the electron heating may depend on the details of the balance between turbulence stabilizing and destabilizing contributions. Once the ion temperature and the toroidal rotation velocity start decreasing in the presence 

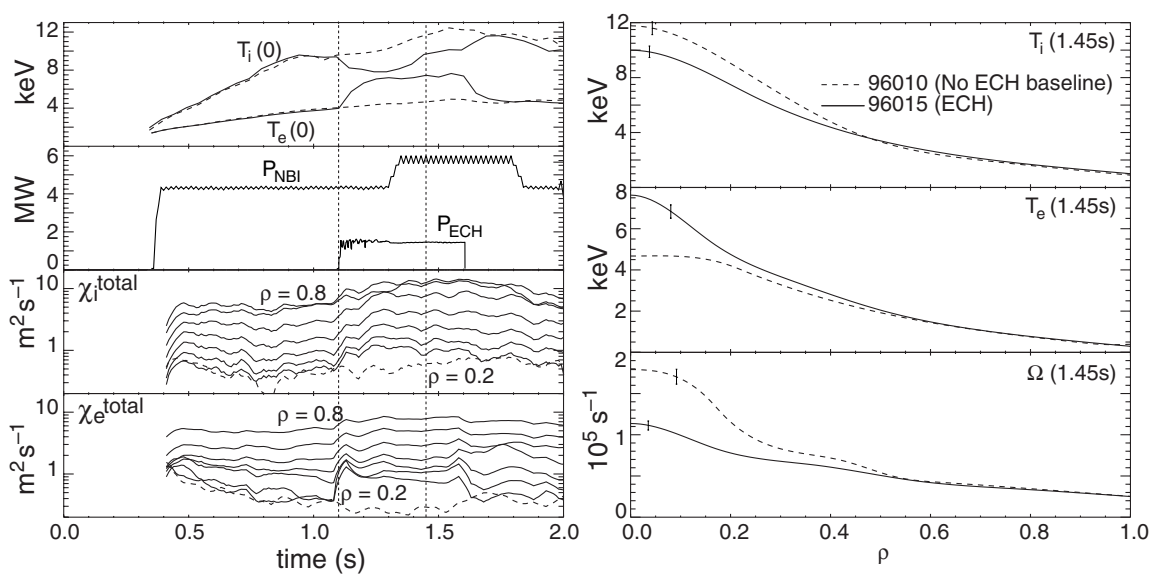

Figure 39. Effect of electron heating by ECRH on an ITB discharge in DIII-D. The time traces of central temperatures, heating powers and inferred heat conductivities are shown. The temperature and toroidal rotation $(\Omega)$ profiles are compared to a reference case without ECRH (from Greenfield et al (1999); copyright in these materials is vested in the International Atomic Energy Agency, Vienna, Austria from which permission for publication must be obtained). In this example additional ECRH increases both the ion and electron thermal heat conduction and the momentum transport.

of ECRH, a further confinement degradation seems to be initiated by the combined effects of $E \times B$ shearing rate reduction, amongst other things due to the drop of $\mathrm{d} v_{\phi} / \mathrm{d} r$, and the rise of the ITG mode growth rate (Greenfield et al 1999), possibly caused by the $T_{\mathrm{e}} / T_{\mathrm{i}}$ increase.

In the experiments reported above pure electron heating in the form of ECRH or FWEH was added together with constant NBI powers. Another method to increase the electron heat flux is ICRF minority heating, which primarily heats the electrons, provided the minority concentration is low enough and the ion temperature is not too high (which would increase the direct coupling of the ICRF waves to the main ion population). In addition to the different heating mechanism, when compared to ECRH and FWEH, ICRF minority heating tends to have broader power deposition profiles and thus lower local heat fluxes. The power deposition depends on the fast minority ion orbits, which can become very large at the low poloidal magnetic field values in the core of negative magnetic shear configurations. The size of these orbits can even result in the loss of the fast particles to the wall.

In JET the combination of NBI and ICRF heating has been used regularly for the formation and sustainment of internal transport barriers in the optimized shear regime (Cottrell et al 1999). In JT-60U dedicated experiments have been performed, in which some of the neutral beam power is replaced by the same amount of ICRF power, keeping the total power constant (Iwase et al 1999). However, exchanging ICRF against NBI power, not only modifies the partition between ion and electron heating powers, but also reduces the particle fuelling, the current drive and the momentum transfer, all three provided by NBI. This complicates the interpretation of such electron heating experiments, as density, current and toroidal rotation profiles may be affected directly, in addition to the influence on the confinement. Nevertheless, it appears that neither in JET (Cottrell et al 1999, Wolf et al 2001b) nor in JT-60U (Iwase et al 1999) does the electron heating by ICRH adversely affect the confinement. At least for JET it has to be added, that the $6 \mathrm{MW}$ of ICRF power, normally coupled to the plasma, is just the level required to reach the critical electron temperature gradient length of $\mathrm{H}$-mode plasmas (Suttrop et al 2001). This may not be sufficient to probe possible increases of the electron heat transport in ITB plasmas, which may exhibit reduced critical values of $L_{T_{\mathrm{e}}}$ (Wolf et al 2001b). 
The confinement degradation, observed in the DIII-D ITB discharges with additional ECRH or FWEH heating resembles the behaviour of low density neutral beam heated H-mode discharges in ASDEX Upgrade, which are critical gradient limited and thus, according to the ITB definition given in section 2.3.3, do not exhibit ITBs (Peeters et al 2001b). Adding central ECRH to these plasmas with weakly coupled electrons and ions and hence $T_{\mathrm{i}}>T_{\mathrm{e}}$ results in a reduction of $R / L_{T_{\mathrm{i}}}$, while that of the electrons stays constant (Wolf et al 2001b). A conceivable interpretation is that the increase of $T_{\mathrm{e}} / T_{\mathrm{i}}$ causes the rise of the critical gradient length of $T_{\mathrm{i}}$, as predicted by ITG mode theories. Similar to high density H-mode discharges, the increase of the central electron heat flux is also accompanied by a substantial drop of the central electron density. In the high density cases this flattening of the density profile following the increase of the central heat flux can be described by a constant ratio of particle to effective heat diffusivity of $D / \chi_{\text {eff }} \approx 0.1$ and the neoclassical Ware pinch for the particle convection (Stober et al 2001b, 2002) In the presence of stiff temperature profiles the increase of $\chi_{\text {eff }}$ and therefore also $D$ is then a consequence of the rise of the central heat flux.

Now, the question remains, as to why some ITB plasmas exhibit such a confinement degradation, when increasing the central electron heating (Staebler et al 1998, Greenfield et al 1999), while others do not (Forest et al 1996, Iwase et al 1999, Wolf et al 2000, Günter et al 2000b, Ikeda et al 2001, Wolf et al 2001b). At first it should be considered, whether in all cases the electron heat flux was sufficiently large to probe the different transport channels. For the electron transport, which always seems to stay above neoclassical, it has been argued that ETG driven turbulence may still exist in ITB plasmas (Stallard et al 1999). Hence, the critical gradient length may be reduced by for instance modifications of the magnetic shear, but could still exist and therefore would result in an increase of the electron heat conduction if the corresponding heat flux is raised. This explanation, however, is not in agreement with the theory by Jenko et al (2000), which for negative magnetic shear predicts a complete suppression of the radially elongated streamers, made responsible for the enhanced electron transport levels. For the ion transport, experimental results from JET indicate that, similar to the above argument for ETG modes, low or negative magnetic shear may only decrease $L_{T_{\mathrm{i}}}$, still retaining the critical gradient behaviour (Baker et al 2001, Wolf et al 2001b) and thus possibly the direct dependence on $T_{\mathrm{e}} / T_{\mathrm{i}}$. In addition, the $E \times B$ shearing rate, although large enough to stabilize ITG modes without central electron heating, could be insufficient to overcome the increased ITG mode growth rate at larger $T_{\mathrm{e}} / T_{\mathrm{i}}$.

As already mentioned, the dependence of the $E \times B$ shearing rate on the poloidal magnetic field may contribute to the ITG mode stability, as a low $B_{\theta}$ in the plasma core increases the $E \times B$ shearing rate. This may explain why the cases where the central electron heating is combined with counter-current drive, which tends to enhance the central shear reversal, i.e. lower $B_{\theta}$, do not show adverse effects of the electron heating. Finally $\alpha$-stabilization, which acts to decrease ETG and ITG driven turbulence as the respective temperature gradients increase, may be essential in achieving $T_{\mathrm{i}} \approx T_{\mathrm{e}}$ in plasmas with ITBs.

So far in this section only examples of ITB plasmas with $T_{\mathrm{i}} \approx T_{\mathrm{e}}$ have been discussed, where, due to the low density, electrons and ions are weakly coupled. In the various tokamak discharge scenarios with internal transport barriers there seem to be only two exceptions to this. The first is the PEP mode (Kamada et al 1991, Tubbing et al 1991, Geraud et al 1994, Takase et al 1996, Baylor et al 1997), in which a reversed magnetic shear configuration with an ITB at high densities is facilitated by the injection of frozen deuterium pellets, followed by strong central heating of the plasma (for a more detailed description see beginning of section 3 ). The PEP mode, although more reactor relevant with respect to the strong collisional energy transfer between electrons and ions, up to now has not been pursued further, partly because of its transient nature. 
The second exception is high density ITB discharge in Alcator C-Mod (Fiore et al 2001, Rice et al 2001). They are distinguished by the development of strong density gradients in the inner half of the plasma cross section. As no evidence of barriers is seen in the electron or ion temperature profiles, these ITBs cannot be characterized by the criterion given in section 2.3.3, which relies on changes of the temperature gradient lengths. The Alcator C-Mod ITBs are obtained in H-mode plasmas with purely Ohmic or off-central ICRF heating. The measurement of the toroidal impurity rotation velocity suggests that, assuming neoclassical poloidal rotation, the ITB formation is associated with a change of the sign of the radial electric field. However, both, the mechanisms which drive this rotation in the Ohmically and ICRF heated plasmas and which cause the ITB formation are unexplained.

Whether the model to describe the density peaking in ASDEX Upgrade H-mode discharges, which links heat and particle transport, is also applicable to the Alcator C-Mod ITBs, in particular with off-axis ICRH, remains to be seen. In ASDEX Upgrade in the presence of stiff temperature profiles, i.e. in the absence of ion or electron temperature ITBs, the density peaking is explained by a reduction of the central heat flux, jointly decreasing the central heat and particle diffusion (Stober et al 2001b, 2002).

\section{Internal transport barrier dynamics}

As presented in the previous sections, ITBs occur under various conditions. Depending on the interplay between the mechanisms driving and stabilizing plasma turbulence, reductions of the ion heat, electron heat or particle transport may appear. The turbulence drive is basically given by the free energy of the gradients of temperature and density (Connor and Wilson 1994), which adjust themselves according to the supplied heat and particle fluxes, and the transport coefficients, which describe diffusion and convection. The heat and particle fluxes are determined by the different heating methods and the fuelling and recycling at the plasma periphery. In a burning fusion plasma the main heat source will be the fast $\alpha$-particles from the fusion reaction between deuterium and tritium, which is also a source of helium ions and a sink for the fuel ions.

In order to form an internal transport barrier, first the conditions for the transport bifurcation from low to enhanced core confinement have to be met, which includes a certain threshold power (Koide et al 1996, Lao et al 1996b, Synakowski et al 1997b, Koide et al 1998a, Greenfield et al 2000a, Fukada et al 2001, Challis et al 2001a). In contrast to the H-mode power threshold, which roughly scales with plasma density, magnetic field strength and plasma dimensions (Ryter et al 2002), the transition to ITBs in general does not seem to follow such a simple description (Fukada et al 2001). Secondly, the ITB once obtained has to be sustained. Similar to the H-mode, ITBs exhibit a hysteresis behaviour (Diamond et al 1997, Synakowski et al $1997 \mathrm{a}, \mathrm{b})$, i.e., the power to sustain them is below the threshold power. Besides, for the steady state sustainment of ITBs, the control of current and pressure profiles to adjust to the desired confinement and stability properties may be required. Another critical issue, especially when considering longer times scales, is the avoidance of undue impurity concentrations in the plasma centre.

\subsection{Internal transport barrier formation and sustainment}

4.1.1. $E \times B$ shearing and magnetic shear. For the formation of internal transport barriers, in general, the interplay between low or negative magnetic shear and sufficiently large $E \times B$ shear flows has been identified to be an essential ingredient (Burrell 1997, Synakowski 1998). The first provides access to the second stability region by stabilizing high- $n$ ballooning modes 
and has a direct stabilizing influence on plasma turbulence, as was recognized already by Kadomtsev and Pogutse (1967). The latter facilitates the stabilization of the residual long to medium wavelength turbulence, mainly driving ion and particle transport. Assuming that the fastest linear growth rate, $\gamma_{\max }^{\operatorname{lin}}$, of the dominant microinstability represents an upper limit of the turbulent drive, the stabilization condition is given by $\omega_{E \times B}>\gamma_{\max }^{\text {lin }}$ (Biglari et al 1990, Waltz et al 1994). The electron heat transport, on the other hand, due to the smaller scale length of the underlying turbulence is not believed to be affected by sheared flows. Thus, negative magnetic shear may be a necessary condition for the formation of ITBs in the electron channel. However, this requirement is moderated by the stabilizing effect of an increasing Shafranov shift ( $\alpha$-stabilization: see sections 2.2 and 3.2).

As outlined by (Synakowski 1998), additional interdependences exist between the magnetic shear and sheared $E \times B$ flows. First, a hollow current profile, i.e. shear reversal, produces a larger Shafranov shift for a given plasma energy, which itself may lead to a reduction of the turbulence (Beer et al 1997). Second, the larger Shafranov shift generates increased pressure gradients in the outer midplane of the plasma and hence larger values of $E_{r}$ and its gradients, which raises $\omega_{E \times B}$. This comes into effect, only if the toroidal plasma rotation is not the dominant contribution to the $E \times B$ shearing rate. Third, in a tokamak plasma the reduction of the magnetic shear corresponds to a larger $q_{0}$ or a lower poloidal magnetic field near the magnetic axis, which again increases $\omega_{E \times B}$. The last argument has been used to explain the confinement improvement in DIII-D plasmas with ITBs and $T_{\mathrm{i}} \approx T_{\mathrm{e}}$ (Forest et al 1996) (see section 3.3).

Figure 40 exemplifies the dependence between magnetic shear and $E \times B$ shearing rate, necessary to form internal ion heat transport barriers in JET optimized shear discharges (Tala et al 2001), which are characterized by monotonic $q$ profiles $(s \geqslant 0)$. Both quantities, $s$ and $\omega_{E \times B}$ are taken at the footpoint of the barrier in the ion temperature profile, i.e. at the maximum of $\mathrm{d}^{2} T / \mathrm{d} r^{2}$, when the ITB emerges. Obviously, $\omega_{E \times B}$, required for the confinement transition, decreases with decreasing magnetic shear, giving evidence for the synergy between magnetic and $E \times B$ shear. This also shows, that at a given $\omega_{E \times B}$ the ITB cannot exist beyond a certain value of magnetic shear. In fact, theoretical models predict the expansion of the ITB exactly to the point, where $\omega_{E \times B}$ no longer exceeds $\gamma_{\max }^{\operatorname{lin}}$ (Kinsey et al 2001). A further lowering of the magnetic shear towards negative values also suggests a reduction of the necessary $\omega_{E \times B}$. Consequently, as the build-up of pressure gradients and plasma rotation, determining $\omega_{E \times B}$, is directly related to the heating power, a reduction of the magnetic shear may serve to decrease the power threshold (Challis et al 2001a). In figure $40(b) \omega_{E \times B}$ is normalized to the maximum linear growth rate of ITG modes, which reduces the scatter of the data, indicating that ITG driven turbulence indeed dominates the anomalous transport prior to the ITB formation.

There are many possibilities for modifying the local value of $E_{r}$ and hence $\omega_{E \times B}$ through their dependence on plasma pressure and rotation velocities. As mentioned in section 2.2.6, many possible feedback loops between the components of $E_{r}$ and their effect on the plasma transport exist (Burrell 1997 and references therein), which may induce confinement transitions. How the components of $E_{r}$ are affected generally depends on the interplay between plasma confinement and the heating method applied. In TEXTOR experiments were performed, in which $E_{r}$ was controlled by external means, namely with electrically biased probes in the plasma edge. The results indicate, that the electric field shear, and not merely $E_{r}$, modifies the confinement (Jachmich et al 1998, Weynants et al 1998). In agreement with the $E \times B$ shearing rate, given by Hahm and Burrel (1995), also the sign of $\partial E_{r} / \partial r$ appears not to be relevant with respect to the decorrelation of the turbulent eddies. In addition, the results of these experiments support the perception, that the increased $E \times B$ shear is the cause of the confinement improvement and not merely the result of the modified confinement properties. In DIII-D the modification of the rotational shear by magnetic braking, using an external coil to 

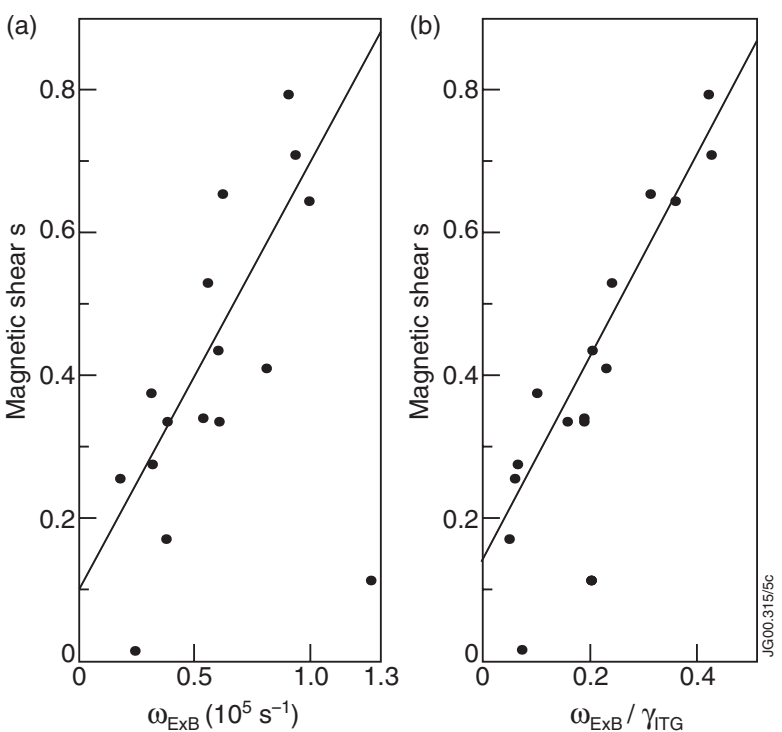

Figure 40. (a) Dependence of magnetic shear, $s$, on the $E \times B$ shearing rate, $\omega_{E \times B}$, for JET optimized shear discharges (from Tala et al (2001)). The quantities are evaluated at the onset of the ITB and at the location of the footpoint of the barrier in the ion temperature profile. $\omega_{E \times B}$ is derived from CXRS measurements of ion density, temperature and toroidal rotation velocity, assuming neoclassical poloidal rotation. Lacking MSE measurements for these discharges, the magnetic shear is inferred from current diffusion calculations, using neoclassical conductivity. (b) Also shown is the dependence on $\omega_{E \times B}$ normalized to a simplified estimate of the maximum linear growth rate of the ITG modes, which reduces the scatter of the data, indicating that ITG modes indeed dominate the anomalous transport prior to the ITB formation. The lines which are drawn through separate the plots into areas of anomalous transport (above the lines) and reduced transport (below the lines).

perturb the internal magnetic field, gives further evidence for this causal connection (La Haye et al 1995, Lao et al 1998).

The influence of the toroidal momentum on ITB confinement has been studied by Synakowski et al (1997a, b, 1999), Greenfield et al (2000a), Shirai et al (2000), Sakamoto et al (2000). In many of the tokamak discharges with ITBs, neutral beam injection is the dominant heating method (Koide et al 1997, Greenfield et al 1999, Bell et al 1999a, Challis et al 2001a, Wolf et al 2001a). If the heating beams are oriented in such a way, that they have a unidirectional toroidal component, the momentum carried by the fast beam ions leads to the toroidal rotation of the bulk plasma. The strength and distribution of this rotation depend on the momentum transport, which is determined by the friction between adjacent layers at different velocities. Experimental observations, that the $v_{\phi}$ profiles roughly resemble those of $T_{\mathrm{i}}$, even in the presence of ITBs (see, for instance, Sips et al (1998), Doyle et al (2000), Challis et al (2001b)), suggest a close link between momentum and ion heat transport.

Unlike in JET, DIII-D and ASDEX Upgrade, the symmetric arrangement of the neutral beam injectors in JT-60U and TFTR allows ITBs to be produced with balanced NBI, where equal powers are injected in opposite directions and hence the applied torque almost cancels to zero (Synakowski et al 1997a, b, Shirai et al 2000). The residual torque is a consequence of the increased orbit losses of the fast particles, injected in the counter-current direction (Shirai et al 2000, Greenfield et al 2000a). Taking this into account, the calculated radial distribution of the toroidal momentum in JT-60U ITB plasmas produced with balanced injection shows central 
values, which are only reduced by about a factor of two (Shirai et al 2000). The total toroidal momentum, integrated over the whole plasma volume, is of course much lower, compared to unidirectional NBI. However, since the shear of $v_{\phi}$ is the decisive quantity affecting the $E \times B$ shearing rate, the more narrow distribution of the toroidal momentum can be beneficial for the suppression of turbulence (Shirai et al 2000, Sakamoto et al 2001). Indeed, in JT$60 \mathrm{U}$ balanced or near balanced injection results in a local notch of $v_{\phi}$ at the ITB position, while unidirectional co- or counter-NBI leads to broad, monotonic radial profiles with smaller gradients. As a consequence the sustainable increase of the core confinement is smaller with unidirectional NBI in JT-60U, although the heating power and its distribution is similar to the case with balanced NBI.

Experiments, performed in TFTR, in which during the course of the discharge the neutral beam heating is changed from balanced to various degrees of co-current injection have highlighted different $E \times B$ shear flow dynamics, involved in the formation and sustainment of ITBs. First, the ERS regime (see section 3.1.3) is established at high heating power (28 MW). Subsequently, the heating power is stepped down to $14 \mathrm{MW}$ (figure 41). Depending on how much torque is applied by an imbalance between co- and counter-injected beams, the ITB

(a)

(d)

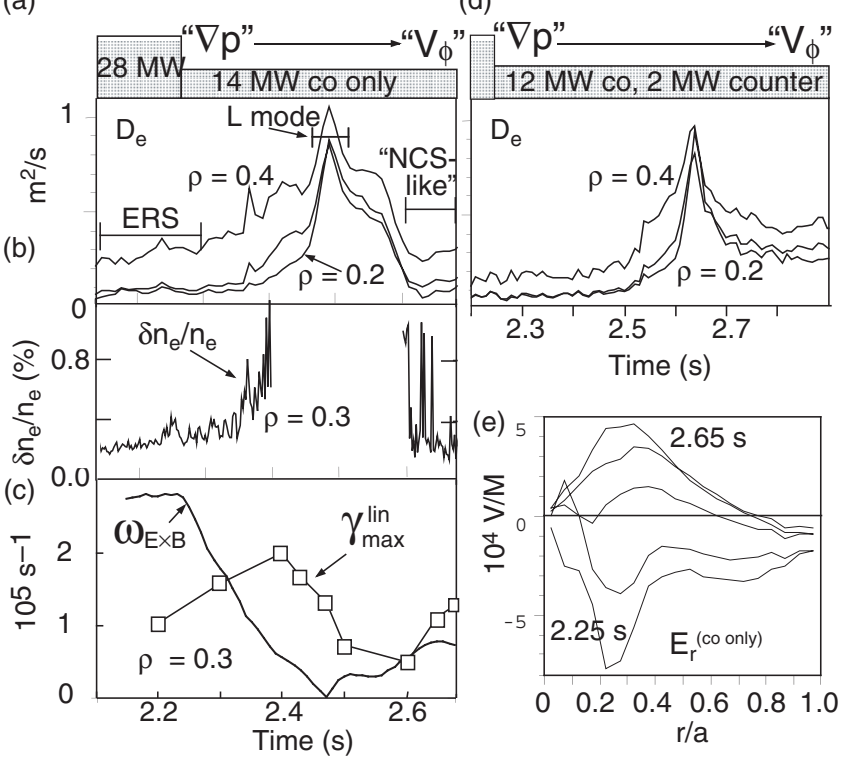

Figure 41. Experiments, performed in TFTR, to study the different $E \times B$ shear flow dynamics, involved in the formation of ITBs, when changing from balanced to co-current NBI (from Synakowski et al (1999)); copyright in these materials is vested in the International Atomic Energy Agency, Vienna, Austria from which permission for publication must be obtained). (a) The particle diffusivity at different radial locations is inferred from measurements of density and temperature, $(b)$ the transient back transition into L-mode confinement is consistent with an increase of the density fluctuations and $(c)$ an $E \times B$ shearing rate, $\omega_{E \times B}$, which clearly drops below the maximum linear growth rate, $\gamma_{\max }^{\operatorname{lin}}$, of the dominating turbulence. $\omega_{E \times B}$ and $\gamma_{\max }^{\operatorname{lin}}$ are evaluated at the foot of the barrier at $\rho=0.3$. $(d)$ The recovery of the confinement depends on the net torque applied, which is modified, replacing some of the co-NBI by counter-NBI. (e) With balanced NBI $E_{r}$ is dominated by the pressure gradient term, resulting in a well at the ITB location. At high toroidal momentum, the well is replaced by a hill at a similar position, which is produced by the $v_{\phi} B_{\theta}$ term. Going from one improved confinement state to the other, the $E_{r}$ profile has to become flat, causing the decrease of $\omega_{E \times B}$ below $\gamma_{\max }^{\operatorname{lin}}$. 
starts to decay at different times (Synakowski et al 1997a, b). The total power is kept constant at this stage, in order to exclude as far as possible other means of turbulence stabilization, such as changes of the Shafranov shift and density gradients. While with balanced NBI the ERS confinement can be sustained even at the lower power, the onset of the confinement degradation is observed earlier the more co-NBI is applied.

However, waiting sufficiently long, the confinement recovers again (Synakowski et al 1999). This behaviour is reflected in the evolution of the particle diffusivity (figure 41(a)), the density fluctuations (figure $41(b)$ ) and the ratio of $E \times B$ shearing rate to the maximum linear growth rate of the turbulence (figure $41(c)$ ). The increase of the radial transport up to L-mode levels is accompanied by an increase of the density fluctuations and is consistent with $\omega_{E \times B}$ dropping below $\gamma_{\max }^{\text {lin }}$. The transient confinement collapse, when changing from an ITB with balanced NBI to an ITB plasma dominated by toroidal rotation is explained by evolution of the $E_{r}$ profile (figure $41(e)$ ). At low toroidal momentum $E_{r}$ is dominated by the pressure gradient term, resulting in a well at the ITB location. At high toroidal momentum, the well is replaced by a hill at a similar position, which is produced by the $v_{\phi} B_{\theta}$ term. Going from one improved confinement state to the other, the $E_{r}$ profile has to become flat in between, causing the decrease of $\omega_{E \times B}$ below $\gamma_{\max }^{\text {lin }}$. In agreement with observations in DIII-D (Rettig et al 1998) and JET (Challis et al 2001b, Budny et al 2002) the confinement improvement is correlated with the applied torque in toroidal rotation dominated ITB plasma. As compared in figures 41 $(a)$ and $(d)$, a reduction of the net toroidal momentum, replacing some of the co-NBI by counter-NBI, leads to a higher transport level in the NCS like phase, which is consistent with the dependence of the $E \times B$ shearing rate on the rotational shear.

In the absence of rotational shear, the mutual amplification between turbulence stabilization, increase of Shafranov shift and increase of $\omega_{E \times B}$ has been suggested as a feedback loop leading to the confinement transition of the ERS mode (Beer et al 1997). The steepening of the pressure gradient results in a rise of the Shafranov shift and at $v_{\phi} \approx 0$ to a increase of $\omega_{E \times B}$, both stabilizing turbulence, which in turn increases $\nabla p$.

Another important difference between the various NBI schemes is the effect on the current profile. NBI in the co-current direction is usually preferred for its smaller orbit losses and, thus, higher heating efficiency. In addition, for conventional current profiles, which are peaked in the plasma centre, the neutral beam current drive in the direction of the plasma current supports this current profile, provided the power deposition of the beams is sufficiently central. However, to sustain reversed magnetic shear profiles, i.e hollow current profiles, counter-current NBI is favourable, as the central beam current drive opposite to the plasma current tends to deplete the central current density (Greenfield et al 2000a). While neutral beam current drive in the cocurrent direction saves magnetic flux and thus helps to extend the possible discharge length, counter-current drive sustains hollow current profiles at the expense of a higher magnetic flux consumption. A way out maybe offered by neutral beam configurations, which combine off-axis deposition with a sufficiently large toroidal component in the direction of the plasma current, providing both the support of hollow current profiles and the reduction of the flux consumption (Gruber et al 1999a, Fujita et al 2001b).

Once the ITB is formed, the expansion of the improved confinement region at constant heating power sometimes happens in discrete steps rather than continuously (Greenfield et al 1999, Günter et al 2001b). These growth events are associated with an abrupt reduction of the core transport, resulting in an increase of the core temperature and rotation velocity and transient reductions of these quantities outside the ITB. The latter results from the drop of the local heat flux just outside the barrier, as at constant heating power the available heat is held up to increase the energy inside the barrier. This effect can be so strong, that in the presence of an H-mode edge, the pedestal pressure at the plasma edge drops below the H-mode threshold, 

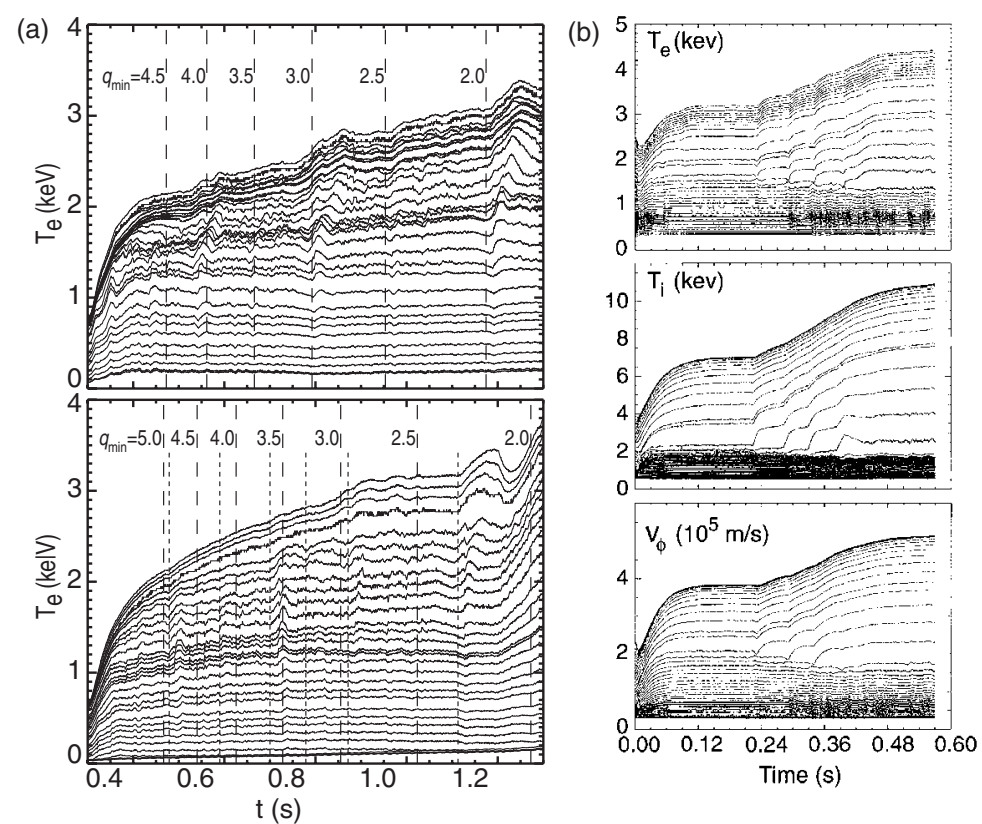

Figure 42. Comparison of (a) measured (from Greenfield et al (1999)); copyright in these materials is vested in the International Atomic Energy Agency, Vienna, Austria from which permission for publication must be obtained) and (b) calculated (from Kinsey et al (2001)) temporal evolution of ITBs. (a) The measurements show time traces of the electron temperature at approximately equally spaced radial locations of two DIII-D discharges. In the examples given, the expansion of the improved confinement region at constant heating power takes place in discrete steps rather than continuously (Greenfield et al 1999, Günter et al 2001b). These growth events often coincide with the time when $q_{\min }$ crosses low order rational values, indicated by the vertical dashed lines. The dotted lines show the growth events, which appear not to coincide with such rational values. A similar behaviour is observed in the ion temperature and toroidal rotation velocity measurements. However, due to their lower spatial and temporal resolution, the growth events are not so clearly resolved. $(b)$ In the theoretical calculation of the thermal and toroidal momentum transport, selfconsistently computing the $E \times B$ shear flow stabilization of the turbulence, the stepwise growth and expansion of the ITB, seen on the traces of electron temperature, ion temperature and toroidal rotation velocity, is well reproduced (Kinsey et al 2001, Staebler et al 2001).

causing a back transition into an L-mode edge. As illustrated in figure 42(a), the growth events often, but not always, coincide with the time when $q_{\min }$ crosses low order rational values.

Many features of transport bifurcations have been reproduced at least qualitatively by various phenomenological models (Diamond et al 1997, Staebler et al 1997a, Fukuyama et al 1998, Newman et al 1998,Parail et al 1999). As sketched in figure 43 for one type of theoretical model (Diamond et al 1997, Newman et al 1998), the driving flux, $\Gamma$, is a non-linear function of the local gradients of density or temperature (for the application to the transition from L- to H-mode see Hubbard et al (2000)). For convenience $\nabla n$ is used in the following discussion, but it should be regarded as synonymous for any other local gradient on which the flux $\Gamma$ may depend. The curve in figure 43 represents one radial position only. Although the principal shape is the same at other radii, the exact values of $\Gamma(\nabla n)$ may differ. Initially, if $\nabla n$ is increased by raising the heating power, the anomalous transport leads to a strong increase of the flux, $\Gamma$. Eventually at the summit, the height of which is determined by the various turbulence stabilization mechanisms and their dependence on $\Gamma$ and $\nabla n$, the confinement transition is triggered. Subsequently, any further increase of $\Gamma$ with $\nabla n$ is determined by 


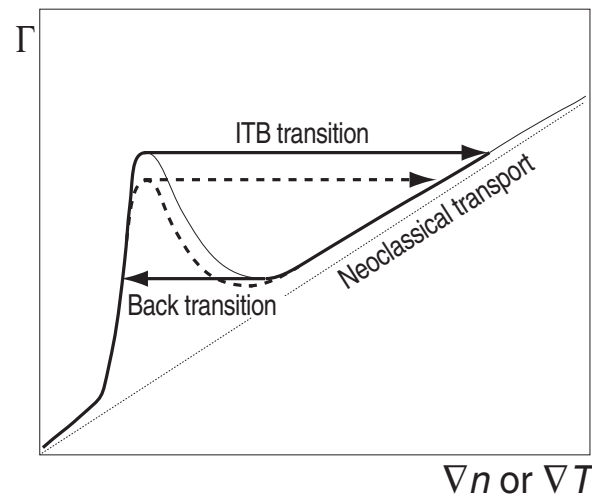

Figure 43. Qualitative dependence of the driving flux, $\Gamma$, on the local gradients, e.g. $\nabla n$ or $\nabla T$. The region of anomalous transport is characterized by a strong increase of $\Gamma$ with $\nabla n$ or $\nabla T$. In the ITB region, the lower limit may be given by neoclassical transport. The $\Gamma$ at the confinement transition to the ITB is above the value of the back transition. The height of the summit, at which the forward transition takes place, is determined by the various turbulence stabilization mechanisms and their dependence on $\Gamma$ and $\nabla n$ or $\nabla T$. A lower power threshold (- - -) may be, for instance, caused by transient trigger events due to MHD instabilities.

neoclassical transport, provided the turbulence responsible for the anomalous transport is completely suppressed. This may be true for the ion heat and particle transport, but generally not for the electron heat conduction. Reducing the heating power again, the back transition to low confinement takes place at the local minimum of $\Gamma(\nabla n)$. The heating power, corresponding to this value of $\Gamma$, is lower than the one at the forward transition, which suggests a hysteresis behaviour of the ITB power threshold.

It is interesting to note, that the theoretical model by (Diamond et al 1997) predicts an unfavourable scaling of the ITB power threshold with density. Both the lower $T_{\mathrm{e}} / T_{\mathrm{i}}$ and the positive dependence of the turbulent mode growth rate on the density profile 'peakedness', suggest that the optimum plasma conditions to achieve the formation of an ITB, are low density, hot ion plasmas. Although this is in agreement with the experimental experience (Koide et al 1996, Fukada et al 2001), other factors may contribute to the difficulties in obtaining internal transport barriers at high densities (see section 5). Besides, the dependence on density is rather complex, as sufficiently large gradients may stabilize turbulence again (Beer et al 1997).

The stepwise growth and expansion of the ITB results from the additional dependence of the $\Gamma(\nabla n)$ curves on the plasma radius. The jumps of the confinement originate at the locations where the plasma is marginally stable to the turbulence. In a more quantitative theoretical approach, which includes the dependence of the turbulence growth rates on the local plasma parameters, the energy and momentum transport is calculated (Kinsey et al 2001, Staebler et al 2001). Simulating the thermal and toroidal momentum transport, while self-consistently computing the $E \times B$ shear flow stabilization of the turbulence, quite a good agreement is attained between calculation and measurement (figure $42(b)$ ). Here, the transport bifurcations are explained by the effect of the $E \times B$ shearing. However, as indicated by the experimental data shown in figure $42(a)$, other possible bifurcation mechanims may be based on transport modifications, caused by $q_{\text {min }}$ passing through low order rational values (see below).

The influence of the toroidal rotational shear on radial transport has been discussed in the paragraphs above. On the other hand, the pressure gradient, which enters in the radial electric field, is closely linked to the poloidal plasma rotation via the diamagnetic flow and the neoclassical poloidal rotation (Kim et al 1991, 1994, Staebler et al 1997b). However, in 
agreement with measurements (Bell and Synakowski 2000,Meister et al 2001) the neoclassical poloidal rotation is generally quite small, as the toroidal geometry leads to a strong damping of $v_{\theta}$ (see also section 5.3). While $v_{\phi}$ can easily reach values of several hundred $\mathrm{km} \mathrm{s}^{-1}$, in general, $v_{\theta}$ is below $5 \mathrm{~km} \mathrm{~s}^{-1}$. In connection with the formation of ITBs, there are, however, a few known exceptions. In PBX-M the application of ion-Berstein waves resulted in the transition to an improved core confinement regime, called the core H-mode (LeBlanc et al 1995). This type of ITB is believed to be caused by the shear in the poloidal plasma rotation, generated directly by the electromagnetic waves in the vicinity of the absorption layer (LeBlanc et al 1999). Another example is the transition to the ERS mode in TFTR, which is often accompanied by a spontaneous, shortlived and very localized precursor like excursion of the poloidal rotation (Levinton et al 1998, Bell et al 1998a). In a single documented case this has also been observed prior to the formation of an ITB in ASDEX Upgrade (De Pena-Hempel et al 1998). Such bursts of poloidal rotation, producing large values of $\mathrm{d} v_{\theta} / \mathrm{d} r$, indicate trigger events, which transiently reduce the ITB power threshold considerably (dashed path in figure 43). One possible explanation is based on the reduction of the turbulent viscosity due to $E \times B$ velocity shear, resulting in a localized jet of $E \times B$ flow (Staebler 2000).

4.1.2. Role of low order rational q values. While in the theory by Staebler (2000) no special role is attributed to rational $q$ values, many experiments suggest, that, in the presence of an evolving current profile, ITB transitions are triggered, when low order rational $q$ values emerge as $q_{0}$ or $q_{\text {min }}$ decrease (Koide et al 1994, Bell et al 1996, Greenfield et al 1999, Neudatchin et al 1999, Joffrin et al 2001, Günter et al 2001a, Joffrin et al 2002a). Although different in its appearance, further evidence for a link between transport and rational $q$ values is given by the RTP results (figure 12 in section 2.3.1), which describe the electron heat transport by the coexistence of a multitude of transport barriers in the proximity of rational $q$-surfaces (Hogeweij et al 1998).

In JET optimized shear plasmas, which are characterized by low but positive magnetic shear, the ITBs are observed to form at and subsequently follow the location of low order rational $q$ values (Cottrell et al 1998, Joffrin et al 2002a). This is explained by the coupling of kink modes at the plasma edge to internal $q=1, q=2$ or $q=3$ surfaces, there destabilizing tearing modes. Hence, the underlying correlation of the ITB formation time, is with the occurrence of the external MHD activity (figure 44(a)) and the corresponding rational edge $q$ values (figure 44(b)). The variation of the ITB formation time is achieved by changing the $q$ profile evolution, modifying the timing of the auxiliary plasma heating during the current ramp, the current ramp-up rate and the magnetic field. Now, the coupling of external and internal MHD modes involves two aspects, which can lead to an increase of the $E \times B$ flow shear, acting as a trigger for the confinement transition. First, the local confinement degradation caused by the magnetic island of the internal tearing mode results in perturbations of the gradients of temperature, density and rotation velocities. Second, the differential rotation between the internal and external MHD modes in conjunction with their coupling can lead to magnetic braking of the plasma rotation at the inner $q$-surface (Hender et al 1992, LaHaye et al 1994). To trigger an ITB by these mechanisms, the corresponding edge and core $q$ values have to coincide, which generally means that the respective $q$ value in the core has to occur first, as in an evolving $q$ profile the MHD activity associated with a certain $q_{\text {edge }}$ is not necessarily long-lived.

Also, in reversed magnetic shear JET discharges the ITB formation is closely related to low order rational $q$ values (Joffrin et al 2001). In contrast to the optimized shear plasmas, the ITBs in the negative shear cases emerge, when $q_{\text {min }}$ touches a rational value, but without any obvious signs of the involvement of MHD activity at the edge or in the core. Another difference 

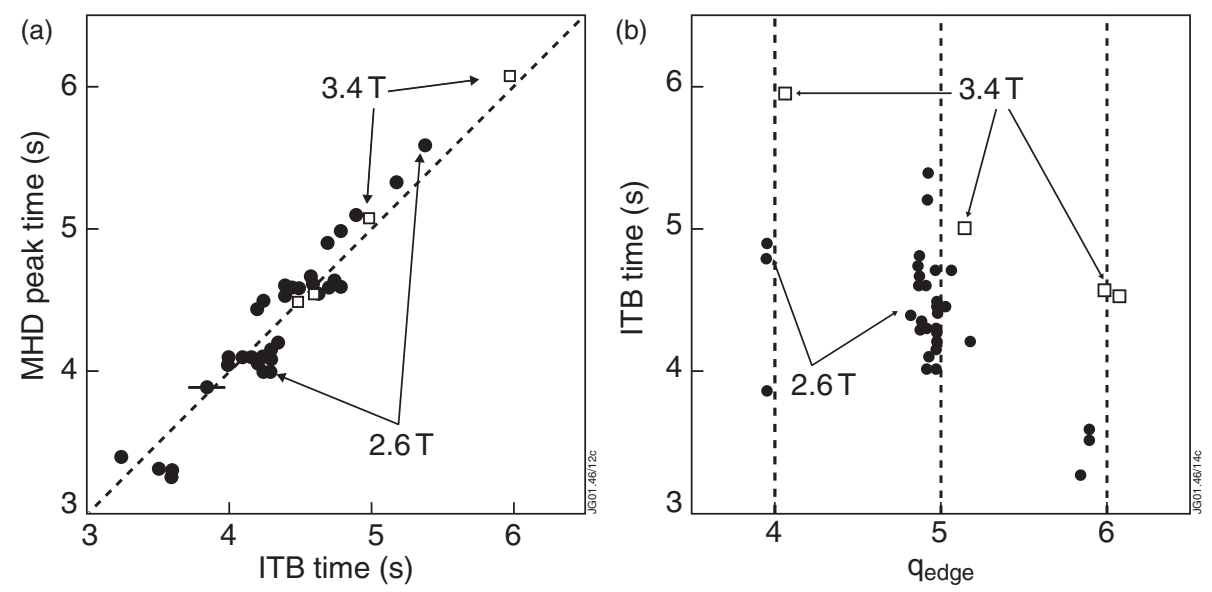

Figure 44. (a) In JET optimized shear plasmas, which are characterized by monotonic $q$ profiles, the time when the ITB emerges in the still evolving $q$ profile is correlated with the appearance of MHD activity at the plasma edge (reproduced from Joffrin et al (2002); copyright in these materials is vested in the International Atomic Energy Agency, Vienna, Austria from which permission for publication must be obtained). The different data points correspond to plasmas, where this $q$ profile evolution has been modified by changes of the timing of the auxiliary plasma heating during the current ramp, of the current ramp-up rate and of the toroidal magnetic field $(2.6$ and $3.4 \mathrm{~T})$. (b) The edge MHD activity corresponds to certain low order rational edge $q$ values.

to the OS ITBs is the much lower power threshold (Challis et al 2001a). This may suggest, that the effect of the magnetic shear on plasma transport, as such, plays an important role in the triggering of the ITB. For instance the rarefaction of the number of low order rational $q$ values at zero magnetic shear, occurring when $q_{\min }$ is close to such values (Garbet et al 2001) (figure 9), could be responsible for a transient reduction of the anomalous transport at the radius of the $q$ profile minimum, which then triggers the transport bifurcation.

In ASDEX Upgrade, fishbone oscillations have been suggested to act as a trigger for the ITB formation (Günter et al 2001a, b). Fishbone oscillations are MHD instabilities driven by fast particles, which, for instance, arise from NBI. In low or reversed magnetic shear plasmas the pressure gradient increases with each such fishbone burst in the vicinity of the corresponding resonant $q$-surface, resulting in a rise of the plasma energy in the core similar to the ITB growth events, described above. A possible explanation for this behaviour is the redistribution of the fast particles by the fishbone instability, leading to a current across the rational surface. This radial current gives rise to a sheared poloidal rotation, resulting in a sheared radial electric field with a shearing rate comparable to the the growth rate of ITG modes. As the poloidal plasma rotation is damped by neoclassical effects, a sufficiently high repetition rate of the fishbone bursts is required to trigger an ITB.

In JET, this trigger mechanism does not seem to apply, possibly due to the lower fast particle pressure. In JET experiments, aiming to reproduce ASDEX Upgrade plasmas with high fishbone activity (Wolf et al 1999b), the fishbone oscillations merely follow the ITB formation (Joffrin et al 2002b). The requirement for a sufficiently large fast particle pressure to destabilize the fishbone instability can also be viewed as a kind of power threshold for the ITB formation, the relevance of which depends on whether other trigger mechanisms come into effect at lower power.

It has been shown, that internal barriers of the ion heat transport, in particular, are formed and exist under many different conditions, determined by the interaction between the various possible turbulence excitation and damping mechanisms. ITBs have been combined with both 
monotonic $q$ profiles and negative central magnetic shear. In both cases the location of the ITB often follows certain positions in the $q$ profile. In case of monotonic shear, this is usually the rational $q$ value at which the ITB was triggered (Koide et al 1994, Cottrell et al 1998, Joffrin et al 2002a), although counter examples exist which do not show a consistent relationship between the early ITB evolution and the details of the current profile (Greenfield et al 1999). Figure 45 illustrates the correlation of ITB position and the radius of the $q=2$ surface for a JET OS discharge. For reversed magnetic shear, it is the radius of the $q$ profile minimum, $\rho_{q_{\min }}$, which is often related to the ITB position (Fujita et al 1998, Wolf et al 2001a). Figure 46

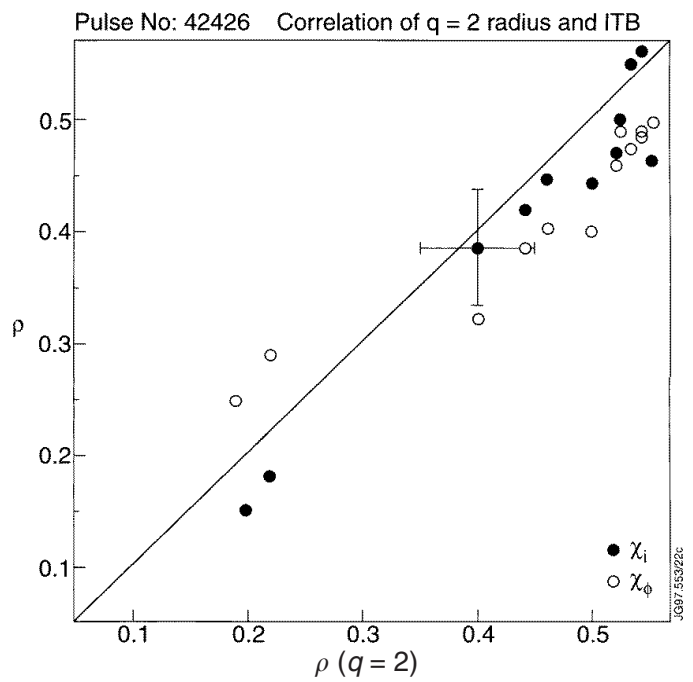

Figure 45. Correlation between the ITB footpoints of ion thermal conductivity, $\chi_{i}$, and angular momentum diffusivity, $\chi_{\phi}$, with the radius of $q=2$ surface in a JET optimized shear discharge (from Cottrell et al (1998)). The footpoint of an ITB is defined as the maximum of the second derivative of the respective quantity.

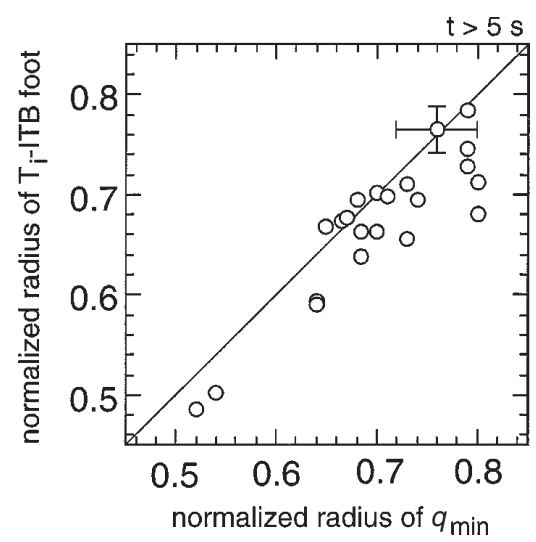

Figure 46. Correlation between the ITB footpoint with the radius of the $q$ profile minimum in JT-60U reversed shear discharges (from Fujita et al (1998); copyright in these materials is vested in the International Atomic Energy Agency, Vienna, Austria from which permission for publication must be obtained). Here, the footpoint of the ITB is defined as the maximum of $\mathrm{d}^{2} T_{\mathrm{i}} / \mathrm{d} r^{2}$. 
shows this correlation in the case of JT-60U reversed magnetic shear discharges, covering a wide range of ITB radii.

However, the analysis of the details of temperature and current profiles in JET reveals that after $q_{\text {min }}$ reaches 2 and continues to decrease, it is possible that even two ITBs exist simultaneously, located at the two $q=2$ surfaces present in the reversed magnetic shear profiles (Joffrin et al 2001, Hender et al 2002). This means, that $q_{\mathrm{min}}$ reaching 2 may trigger the ITB, while the condition for its sustainment is linked to $q$ being rational rather than the zero magnetic shear.

An additional effect, which relates the ITB to a certain position in the $q$ profile has been discussed above. Theory and experiments indicate, that the steady state position of the ITB is determined by $\omega_{E \times B}$ exactly balancing the growth rate of the dominating turbulence (Kinsey et al 2001, Tala et al 2001). Considering the turbulence stabilizing influence of magnetic shear and assuming a constant $E \times B$ shearing rate, this corresponds to a fixed value of magnetic shear (Tala et al 2001) (figure 40). Hence, the location of the ITB would be attached to a certain magnetic shear value.

To add to the complexity of the relationship between the $q$ profile and the formation and evolution of internal transport barriers, the effect of the bootstrap current also has to be considered. In particular, for reversed magnetic shear the bootstrap current also tends to produce a correlation between the ITB position and $\rho_{q_{\min }}$, as the peak of the bootstrap current distribution is basically located at the maximum of the pressure gradient (see section 1.3). Depending on the details of the link between ITB position, $\rho_{\mathrm{ITB}}$, and $q$ profile, the bootstrap current may lead to non-stationary conditions especially for large bootstrap current fractions. Assuming a narrow bootstrap current distribution with the maximum at the ITB position, the ITB tends to shrink for $\rho_{\text {ITB }}<\rho_{q_{\min }}$ and to expand for $\rho_{\text {ITB }}>\rho_{q_{\min }}$. Theoretical models, simulating the evolution of energy, particle content and current profile self-consistently predict that small deviations may be compensated by external current drive (Moreau and Voitsekhovitch, 1999, Voitsekhovitch and Moreau 2001). However, considering the limited current drive efficiency of such techniques and the desire to keep the recirculating power in a fusion reactor low, a coarse mismatch between bootstrap current and the current profile necessary to sustain the ITB has to be avoided.

Another circumstance, which should be considered, when assessing the observed correlation between $\rho_{\text {ITB }}$ and certain points in the $q$ profile, is the slow temporal evolution of both the location of the transport barrier and the current profile, after the ITB has been triggered at some low order rational value of $q$. In the absence of MHD instabilities, changes of the current profile are determined by the neoclassical current diffusion time (see section 3). For example, in an ASDEX Upgrade plasma with $5 \mathrm{keV}$ electron temperature a current perturbation at mid-radius takes about $2.5 \mathrm{~s}$ to diffuse to the plasma centre (Wolf et al 1999b). As the current diffusion or skin time scales with $a^{2}$, in larger tokamaks it can easily exceed the discharge duration.

The observed expansion of ITBs, on the other hand, is also rather slow (Rettig et al 1998). This is in accord with the theoretical considerations, which are illustrated in figure 43 and which postulate that the movement of the ITB, $\rho_{\mathrm{ITB}}(t)$, is governed by the transport rate inside the barrier (Diamond et al 1997, Newman et al 1998), which can be as low as that for the neoclassical level. The change of the barrier position with time is described by $\rho_{\text {ITB }}(t) \approx \sqrt{\left(P-P_{\text {crit }}\right) / P_{\text {crit }}} \sqrt{\chi t}$, where $\chi$ is the low transport coefficient inside the barrier, $P_{\text {crit }}$ the local critical power threshold for the transport bifurcation and $P$ the local heating power supplied (Diamond et al 1997). Thus, depending on how much $P$ exceeds $P_{\text {crit }}$, the timescale for the expansion of an ITB can be of the order of the current diffusion time. Consequently, the ITB and the $q$ profile can develop in such a way, that $\rho_{\text {ITB }}$ matches the radius of a certain $q$ value, without the necessity to tie both by transport effects. 
However, as mentioned above, in JET reversed magnetic shear discharges two simultaneous ITBs have been observed, located in the positive and negative magnetic shear regions close to the two $q=2$ surfaces (Joffrin et al 2001,Hender et al 2002). As $q_{\text {min }}$ decreases below 2, the two ITBs move in opposite radial directions, which at least in this case, contradicts the assumption that the correlation between certain $q$ or magnetic shear values and the ITB position is simply a consequence of similar time scales for current diffusion and ITB movement.

\subsection{Impurity transport}

For the portability of discharges with ITBs to a burning fusion plasma the avoidance of impurity accumulation in the central regions of the plasma is of utmost importance. Impurities in the plasma centre of a fusion plasma are harmful mainly for two reasons. At temperatures present in the core of a fusion plasma low to medium- $Z$ impurities are fully ionized. Hence, their main effect is to dilute the plasma fuel ions, reducing the fusion reaction rate. High- $Z$ impurities, on the other hand are only partially ionized, resulting also in enhanced radiation losses. Sources of impurities are plasma wall interactions and in a burning fusion plasma the helium ash from the fusion reaction itself. Considering the high energy and particle confinement (Efthimion et al 1998, 1999, Takenaga et al 1998) in ITB plasmas, there is a concern that the corresponding impurity transport may be incompatible with the reactor requirements (Efthimion et al 1998, Becoulet et al 2001a).

The theory of neoclassical impurity transport (Hinton and Hazeltine 1976, Hirshman and Sigmar 1981) predicts that the radial distribution of the impurity density depends on the charge number $Z$. In the absence of temperature gradients, the main ion density gradients ${ }^{14}$ of the main or dominant ion species imply a concentration of impurities towards the magnetic axis, which increases with $Z$ (Wesson 1997):

$$
n_{Z}(0)=\left(\frac{n_{\mathrm{I}}(0)}{n_{\mathrm{I}}(r)}\right)^{Z} n_{Z}(r),
$$

where $n_{Z, \mathrm{I}}(0)$ and $n_{Z, \mathrm{I}}(r)$ denote the densities of the main ion and impurity species at the magnetic axis and some minor radius, $r$, respectively. For any peaking of the main ion density profile, i.e. $n_{\mathrm{I}}(0)>n_{\mathrm{I}}(r)$, the central impurity density would rise with the peaking factor to the power of $Z$. This potentially serious problem is counteracted by an effect known as temperature screening, which reduces the inward directed drift velocity of the impurity ions.

While in some experiments no such $Z$ dependence of the impurity transport was seen (Marmar et al 1982, Giannella et al 1994, Mattioli et al 1998), others do confirm this behaviour at least qualitatively (Ida et al 1987, Rapp et al 1997, Dux et al 1999). The ambiguity of the results may be attributed to other mechanisms, which add to the neoclassical transport, such as plasma turbulence and MHD instabilities. In fact, for the controlled expulsion of impurities, relaxation oscillations, like the sawtooth instability or ELMs, are of particular importance (Dux et al 1999).

Recent experimental results confirm the tendency of impurity accumulation for the steep density gradients present in many plasmas with internal transport barriers (Zastrow et al 1999, Chen et al 2001, Dux et al 2001, Doyle et al 2001, Giroud et al 2001). The profile evolution of impurity densities together with the electron density and ion temperature of a JET reversed shear discharge is shown in figure 47. As the ITB of both $T_{\mathrm{i}}$ and $n_{\mathrm{e}}$ build up, the densities of the impurities carbon, neon and $\mathrm{Ni}$ increase in the plasma core, with the strongest peaking observed for the impurity with the highest $Z$, namely Ni. Consistent with neoclassical

\footnotetext{
${ }^{14}$ By density and temperature gradients the normal situation is meant, where $\nabla n$ and $\nabla T$ are negative.
} 


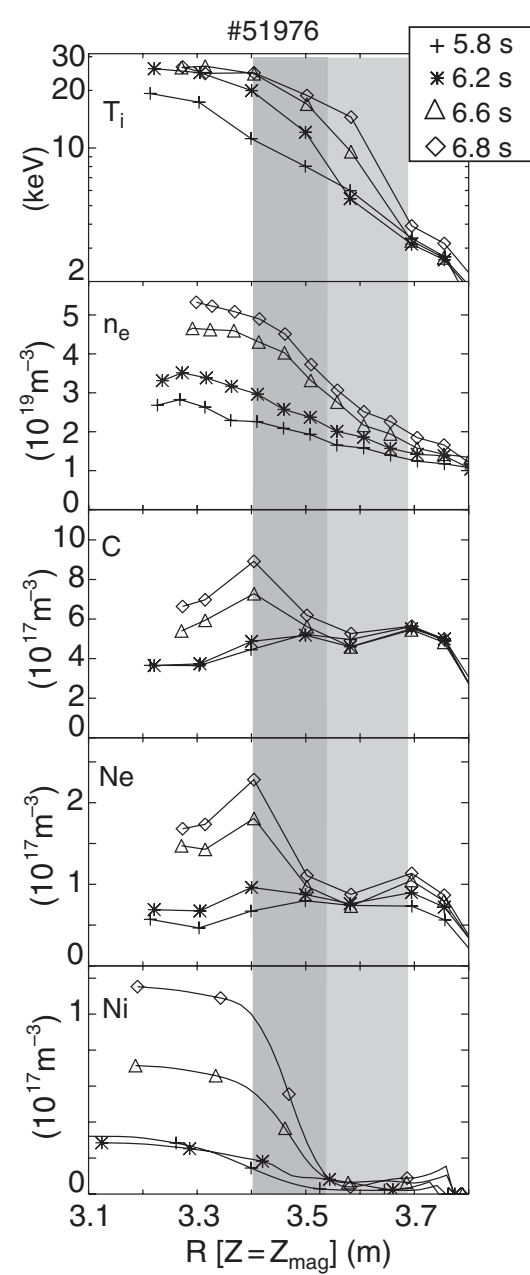

Figure 47. Temporal profile evolution of impurity densities of carbon, neon and nickel, together with the electron density and the ion temperature of a JET reversed magnetic shear discharge (from Dux et al (2001)). As the ITBs of both $T_{\mathrm{i}}$ and $n_{\mathrm{e}}$ build up, the impurity densities increase in the plasma core, with the strongest peaking observed for the impurity with the highest $Z$, namely $\mathrm{Ni}$. Consistent with neoclassical considerations, the temperature screening in the regions of maximum $\nabla T$ inhibits impurity accumulation, while the regions of maximum $\nabla n$ exhibit the strongest increase of the impurity densities.

considerations, the temperature screening in the regions of maximum $\nabla T$ inhibits impurity accumulation, while the regions of maximum $\nabla n_{\mathrm{I}}$ exhibit the strongest increase of the impurity densities. Since, at the final stage, the location of the strongest density gradient overlaps with the region where the temperature plateau has formed, the temperature screening becomes ineffective, resulting, in particular, in high central Ni concentrations. Under such conditions central impurity concentrations corresponding to a central $Z_{\text {eff }}$ of up to seven and a deuterium dilution of up to $20 \%$ have been observed, which actually produced a radiation collapse.

Besides neoclassical diffusion and convection, the latter of which, depending on the profiles of temperature and main ion density, can be inward directed, anomalous diffusion helps to expel impurities. However, in ITBs the decrease of the heat conduction seems to be inherently 
linked to the decrease of main ion and impurity diffusion. This tends to increase the main ion density peaking, although a simultaneous increase of the impurity concentration counteracts such a trend, since at a given electron density each impurity ion replaces $Z$ deuterons or tritons. As has been discussed in section 2.2.4, the increase of $Z_{\text {eff }}$ itself can also result in a reduction of the anomalous transport, which in turn may lead to higher impurity levels by increasing the density profile peaking and $Z_{\text {eff }}$.

To prevent impurity accumulation in ITB plasmas, in addition to minimizing possible impurity sources, limiting the density peaking is an important factor. In JET reversed magnetic shear ITB plasmas the strong density peaking in the absence of MHD instabilities and strong anomalous transport processes seems to be one of the main causes for impurity accumulation. One possibility, to maintain internal barriers of the thermal ion heat transport and simultaneously decrease the density peaking, may be the reduction of the magnetic shear reversal. The comparison between reversed magnetic shear with weakly positive (optimized) magnetic shear ITBs in JET indicates that increasing the magnetic shear lessens the tendency for impurity accumulation. For neon, the impurity convection actually reverses sign from inward to outward directed, when going from negative to positive central magnetic shear (Giroud et al 2001).

For the exploitation of the potential steady state properties of plasmas with internal transport barriers, an operational regime has to be found combining good energy confinement with a particle confinement, which prevents central impurity accumulation. This does not rule out low particle transport in general, but requires conditions where the impurities do not supplant the fuel ions.

\subsection{MHD stability}

A prerequisite for the formation of many types of internal transport barriers is not only the achievement of low heat conduction or particle diffusion, but also the avoidance of certain MHD instabilities, which contribute to the convective transport and thus limit the possible confinement improvement.

Tailoring the current profile to fulfil these MHD stability requirements, both the magnetic shear and certain low order rational $q$ values play a decisive role, similar to the requirements for transport reductions. First, low or negative magnetic shear opens access to the second stability region avoiding the pressure limit given by high- $n$ ballooning modes. Figure 48 shows, that the high central pressure of a DIII-D reversed magnetic shear discharge is consistent with the calculated access to the second stability regime, which is only restricted in the outer regions of the plasma where the magnetic shear is positive (Rice et al 1996b). Second, staying above certain $q$ values avoids MHD instabilities, frequently observed with conventional $q$ profiles, such as the sawtooth instability, which is associated with the $q=1$ surface, or the so-called neoclassical tearing mode (NTM) (Chang et al 1995, Gates et al 1997, LaHaye and Sauter 1998 , Guenter et al 1998), associated with $q=3 / 2$. The latter also cannot form in regions of negative magnetic shear.

However, the strong pressure peaking which develops in ITB plasmas partly as a consequence of the improved stability properties, results in the occurrence of other MHD stability limits which, in particular, in conjunction with low or negative magnetic shear, are difficult to avoid or control. $q$ profiles with low or negative magnetic shear give rise to a variety of additional MHD phenomena, which include both ideal and resistive MHD instabilities ${ }^{15}$,

${ }^{15}$ Ideal MHD modes are instabilities which would occur even if the plasma were perfectly conducting, while resistive MHD modes depend on the finite resistivity of the plasma (Wesson 1997). Ideal modes show much higher growth rates of the magnetic field perturbation. In contrast, the growth of resistive modes is governed by the relatively slow time scale, required for redistributing the magnetic field structure. MHD instabilities are characterized by poloidal 


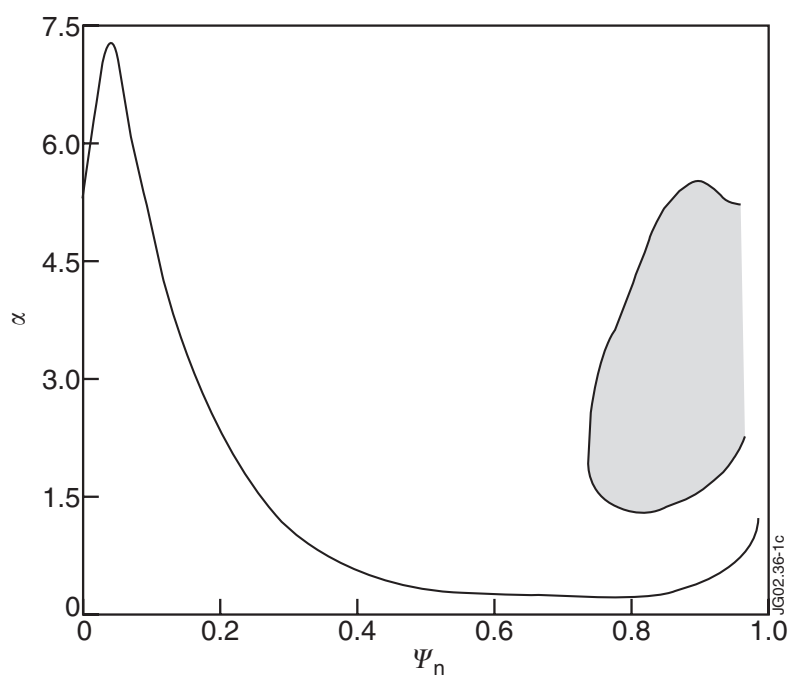

Figure 48. Ballooning stability diagram for a DIII-D reversed magnetic shear discharge (reproduced from Rice et al (1996b); copyright in these materials is vested in the International Atomic Energy Agency, Vienna, Austria from which permission for publication must be obtained). The normalized pressure gradient, $\alpha$, is plotted against the normalized flux, $\psi_{\mathrm{n}}$. Note, that using $\psi_{\mathrm{n}}$ on the abscissa (rather than $\rho=\sqrt{\psi_{\mathrm{n}}}$ ) strongly compresses the central plasma region. Only a small region of instability is present at positive magnetic shear (shaded area). The rest of the plasma has access to the second stability regime.

such as the infernal mode (Manickam et al 1987), the double tearing mode (DTM) (Dewar and Persson 1993) and the resistive interchange mode (Glasser et al 1976).

Depending on the location of the ITB, also with respect to the $q$ profile, many of these instabilities are located in the plasma core in the vicinity of the maximum pressure gradient and are often observed to couple to the plasma edge, which usually leads to the termination of the discharge by disruption. As a result the maximum achievable $\beta_{\mathrm{N}}$ in ITBs rarely exceeds two significantly (ITER Physics Expert Groups 1999 and references therein). Besides this adverse effect, however, MHD instabilities, which are spatially restricted, may be helpful in achieving quasi-stationary conditions in providing pressure, density or impurity control.

4.3.1. Infernal and kink modes. The infernal mode is a low- $n$ ideal pressure driven MHD instability, which is destabilized if a large pressure gradient coincides with low magnetic shear. In $q$ profiles with negative magnetic shear in the plasma centre, the value of $q_{\text {min }}$ also plays a critical role (Manickam et al 1997). Originally, the infernal mode refers to a low- $n$ ballooning type instability (Manickam et al 1987). However, under conditions, which destabilize the infernal mode, low- $n$ kink modes are equally important. Often a combination of these instabilities occurs simultaneously in ITB plasmas, which is then termed kink-ballooning or infernal-kink mode.

In JET optimized shear plasmas $(s \geqslant 0)$ the $\beta$-limit is determined by an ideal $n=1$ pressure driven kink mode (Bondeson et al 1999, Huysmans et al 1999). In figure 49 the calculated stability limit is compared to the evolution of the experimental $\beta_{\mathrm{N}}$ and pressure peaking factor, $\beta(0) /\langle\beta\rangle$. The calculated stability limit also includes the effect of the 

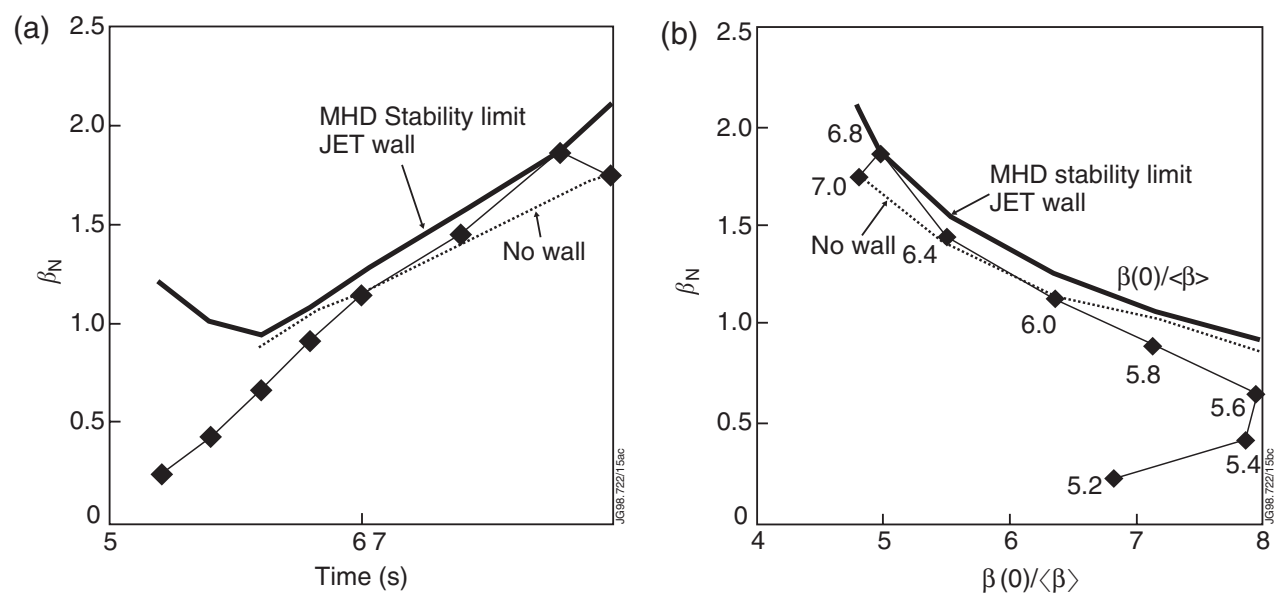

Figure 49. Comparison of calculated MHD stability limit of a JET OS discharge with the experimental $\beta_{\mathrm{N}}$ and pressure peaking factor, $\beta(0) /\langle\beta\rangle$ (from Huysmans et al (1999); copyright in these materials is vested in the International Atomic Energy Agency, Vienna, Austria from which permission for publication must be obtained). The theoretical stability limit has been evaluated with and without the effect of a conducting wall. The experimental discharge trajectories in $(a)$ and (b) are indicated by the rhombuses. The numbers denote the discharge times in seconds. (a) Due to careful control of the central pressure, the experimental $\beta_{\mathrm{N}}$ stays just below the calculated MHD limit. (b) The initially very low critical $\beta_{\mathrm{N}}$ is caused by the strong pressure peaking. Only as the pressure peaking decreases, $\beta_{\mathrm{N}}$ can be raised without making the plasma unstable.

conducting wall, which is small but appears to be significant for the achieved $\beta_{\mathrm{N}}$ later in the discharge, although the error margins of the experimental quantities, involved in the stability calculation and the deduction of the pressure peaking factor, are too large to make a definite statement. Early in the discharge, the extreme peaking of the pressure profile results in a very low critical $\beta_{\mathrm{N}} \approx 1$. Thus, to avoid the disruption of the plasma, it is essential to limit the heating power. During the further course of the discharge, the reduction of the pressure peaking comes along with an increase of the attainable $\beta_{\mathrm{N}}$. Operating just at the theoretical stability boundary results in one of the highest neutron rates obtained in deuterium OS plasmas. This is most efficiently achieved by the feedback control of the heating power on the neutron rate (Sips et al 1998). The drop of $\beta_{\mathrm{N}}$ at $6.8 \mathrm{~s}$ is associated with high frequency MHD modes, which are located near the steep pressure gradient of the ITB (JET Team (presented by F X Söldner) 1997). The simultaneous reduction of $\beta(0) /\langle\beta\rangle$ is caused by the $\mathrm{L}$ - to $\mathrm{H}$-transition of the edge confinement. Consistent with observations in DIII-D (Strait et al 1997) the broader pressure profile in the H-mode increases the stability margin. In the JET case shown here, however, the high frequency MHD modes continue to degrade the core confinement after the H-mode transition.

The beneficial effect of the H-mode transition regarding MHD stability is not observed in ASDEX Upgrade reversed magnetic shear ITBs (Wolf et al 2001a). There, the additional edge bootstrap current of the H-mode pedestal in conjunction with the occurrence of an integer edge $q$ value is made responsible for the destabilization of an external kink mode, which causes the plasma to disrupt, effectively limiting the pressure to $\beta_{\mathrm{N}} \approx 1.7$.

The importance of the pressure profile control is also emphasized in figure 50 for a JT-60U reversed magnetic shear ITB (Fujita et al 1998,Kamada et al 1999a). During the early evolution of the current profile, $q_{\min }$ passes through several integer values, each bearing the risk of a disruption due to MHD instability. Similar to JET, the feedback control of the neutron rate, 


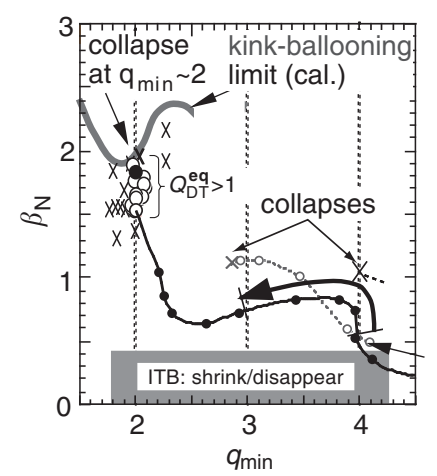

Figure 50. Operational region of reversed magnetic shear ITBs in JT-60U, emphasizing the need of pressure profile control (reproduced from Kamada et al (1999)). $\beta_{\mathrm{N}}$ is plotted against $q_{\mathrm{min}}$, showing several time trajectories and operational points of ITB discharges. During the early evolution of the current profile the operational window lies between too high $\beta_{\mathrm{N}}$ (dashed trajectories), ultimately resulting in $\beta$-collapses, and too low $\beta_{\mathrm{N}}$, leading to a shrinking or disappearance of the ITB (grey region). The reliable achievement of high performance $\left(Q_{\mathrm{DT}}^{\mathrm{eq}}>1\right)$ is best obtained by feedback control of the neutron rate (solid trajectory). The time, when the feedback control is active, is indicated by the arrow. The maximum achievable $\beta_{\mathrm{N}}$ values are consistent with the calculated ideal kink-ballooning stability limit (Ozeki et al 1997).

keeping $\beta_{\mathrm{N}}$ low enough to avoid disruptions, but high enough to sustain the ITB, permits to reach the final $\beta_{\mathrm{N}} \leqslant 2$ at $q_{\min } \approx 2$ reliably. For $q_{\min }$ around 2 the maximum achievable $\beta_{\mathrm{N}}$ is consistent with the calculated ideal $n=1$ kink-ballooning stability limit (Ozeki et al 1997). Depending on the exact location of the maximum $\nabla p$ with respect to the $q$ profile, the character of these MHD modes may change. For $\nabla p_{\max }$ located just inside $\rho_{q_{\min }}$, MHD theory predicts the kink-ballooning mode to be unstable, while shifting $\nabla p_{\max }$ into the positive shear region infernal and high- $n$ ballooning modes may become more relevant (Ishii et al 1998).

The picture in the TFTR (ERS regime) and DIII-D (NCS regime) reversed magnetic shear discharges is similar. While, in general, as predicted by MHD theory, the high- $n$ ballooning modes seem to be stabilized, low- $n$ MHD instabilities remain a problem. In TFTR the $\beta$-limit is explained by low- $n$ infernal-kink like modes, which are destabilized by too large pressure gradients, which develop in the ITB plasmas in the vicinity of zero magnetic shear (Manickam et al 1997, Phillips et al 1996).

In DIII-D stability calculations suggest that either an ideal $n=1$ kink mode (Strait et al 1997) or resistive interchange modes (see below) (Chu et al 1996, Lao et al 1996a, Strait et al 1997) determine the $\beta$-limit for strongly peaked pressure profiles. Both these stability limits show a similar dependence on the pressure profile peaking, as do the experimental data. The increase of the attainable $\beta_{\mathrm{N}}$ with $\beta(0) /\langle\beta\rangle$ is also in quantitative agreement with the JET data (figure 49). Despite the slightly lower critical $\beta_{\mathrm{N}}$ given by the resistive modes, suggesting that they govern the plasma stability, the experimental evidence is not entirely conclusive with regard to which of the two stability limits, ideal or resistive, is the critical one.

As mentioned above, in DIII-D the broadening of the pressure profile, associated with the transition of the plasma edge into H-mode, improves the stability properties. In fact, the combination of ITB, H-mode edge and high plasma current resulted in the transient achievement of record fusion performance ( $Q_{\mathrm{DT}}^{\mathrm{eq}}=0.32$, Lazarus et al 1996). With broader pressure profiles the stability limit follows the linear scaling with the internal inductance, $l_{\mathrm{i}}$, (Strait et al 1997), previously seen in positive magnetic shear plasmas (Taylor et al 1991). Thus, the much higher $\beta_{\mathrm{N}} \approx 4$ observed in these plasmas is attributed to a higher $l_{\mathrm{i}}$ and correspondingly to a lower 
current density at the plasma edge. In contrast to more peaked pressure profiles, the stability of which is related to the $q$ profile in the plasma core, the $l_{\mathrm{i}}$ dependence of the plasmas with broader pressure profiles indicates that the edge current density destabilizes these plasmas.

In ASDEX Upgrade reversed magnetic shear discharges, combined ion and electron heating resulted in internal barriers of the ion and electron heat transport with $T_{\mathrm{e}} \approx T_{\mathrm{i}}$ (Günter et al 2000b, Wolf et al 2000) (see also figure 38 in section 3.3). Sawtooth like oscillations seem to be able to generate quasi-stationary conditions by limiting the peaking of the temperature profile. In contrast to the conventional sawtooth instability, which exhibits mode numbers ( $m=1, n=1$ ) associated with the occurrence of a $q=1$ surface in the plasma, these oscillations are identified as infernal modes with $(m=2, n=1)$ (Guenter et al 2000a, 2001a). Despite the favourable property of these ideal modes to control the central plasma pressure, their usefulness is limited by the tendency to couple to the edge of the plasma as soon as the corresponding $q_{\text {edge }}$ adopts an integer value. The subsequent destabilizing of an external kink mode ultimately causes the plasma to disrupt.

In this context, the so-called barrier localized modes (BLMs) are discussed as a possibility to control the central pressure, density or impurity content in ITB plasmas (Takeji et al 1997, Manickam et al 1999). BLMs are localized at the ITB position, causing minor relaxations of the pressure profile (Takeji et al 1997). Theoretical modelling of JT-60U and TFTR discharges shows that an $n=1$ ideal MHD instability is the likely cause for the observed burst like behaviour near the internal transport barrier (Manickam et al 1999). However, the benign character of BLMs critically depends on the $q$ profile. To prevent BLMs from changing into dangerous global modes, $q_{\mathrm{min}}$ and $q_{\text {edge }}$ must not adopt integer values simultaneously. However, already arbitrary but low $q_{\text {min }}$ in conjuction with an integer $q_{\text {edge }}$ can give rise to global MHD instability, which is also supported by the ASDEX Upgrade observations discussed above.

In summary, the ideal low- $n$ instabilities, which are global in nature, represent a hard limit to the achievable plasma $\beta$. Their avoidance requires the careful control of current and pressure profiles. In general, the limitation of the pressure profile peaking ameliorates the rather low $\beta$-limit. However, its seems difficult to get sufficiently beyond $\beta_{\mathrm{N}}=2$. A possible solution to this problem could be the introduction of a conducting wall close to the plasma periphery. The effect of the wall is to decrease the growth rate of the radial displacements, caused by the ideal instability, to a level where they can be counteracted by external saddle coils (Fitzpatrick and Jensen, 1996). While toroidal plasma rotation is predicted to support the effect of a conducting wall (Bondeson and Ward 1994, Ward and Bondeson 1995), this does not improve the situation in a burning fusion plasma with little or no rotation.

4.3.2. Double tearing modes. The tearing mode is the resistive form of the kink instability (Wesson 1997). While the kink mode is associated with a radial displacement of the flux surfaces, the tearing mode involves the formation of magnetic islands. For the occurrence of double tearing modes, i.e., the destabilization of two coupled tearing modes with the same mode numbers but at different radial locations, two rational magnetic surfaces with the same $q$ value in close proximity are necessary (Dewar and Persson 1993). This is only possible with non-monotonic $q$ profiles.

In conjunction with reversed magnetic shear $q$ profiles, often used for establishing internal transport barriers, DTMs have been observed in various tokamak experiments (Chang et al 1996, Buratti et al 1997, In et al 2000, Günter et al 2000a). The DTM first occurs when in an evolving $q$ profile $q_{\min }$ becomes integer. In many cases this takes place at $q_{\text {min }}=2$. As $q_{\text {min }}$ continues to drop, two $q=2$ surfaces develop, destabilizing ( $m=2, n=1$ ) DTMs. The coupling of these modes basically destroys the confinement in the region in between. The ASDEX Upgrade discharges shown in figure 38 (Wolf et al 2000), in fact, suffer from such a 


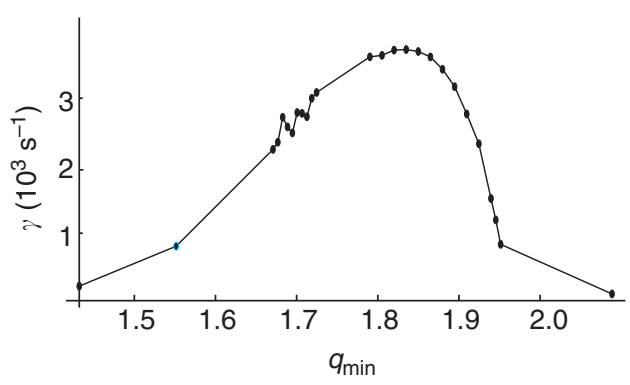

Figure 51. Dependence of the calculated ( $m=2, n=1)$ DTM growth rate on $q_{\min }$ for an ASDEX Upgrade reversed magnetic shear ITB discharge (reproduced from Wolf et al (2000)). First the growth rate increases as $q_{\text {min }}$ drops below 2 . Corresponding to an increase in the distance between the two rational surfaces, the growth rate decreases again, which is consistent with the observed disappearance of the DTM. In the calculations the stabilizing influence of the differential plasma rotation has not been taken into account (Günter et al 2000a).

confinement collapse when $q_{\min }$ reaches 2 . The fast recovery is explained by the increase in the distance between the two $q=2$ surfaces, as $q_{\text {min }}$ decreases further (Wolf et al 2000, Günter et al 2000a). Figure 51 illustrates this effect of $q_{\min }$ on the DTM stability. Besides increasing the distance between the two rational surfaces, also the perpetuation of a strong differential toroidal plasma rotation decreases the susceptibility to DTMs. Hence, a large amount of NBI in the toroidal direction is expected to have a beneficial influence.

An additional effect, specific to $q$ profiles with negative magnetic shear, is the reversal of the influence of the perturbed neoclassical bootstrap current at the location of the magnetic island. For positive magnetic shear, this reduces the tearing mode stability, leading to the occurrence of the neoclassical tearing mode (Chang et al 1995, Gates et al 1997, Zohm et al 1997, LaHaye and Sauter 1998, Guenter et al 1998). For negative magnetic shear, in contrast, the bootstrap current perturbation is theoretically predicted to have a stabilizing effect on the tearing mode (Yu and Günter 1999). For ASDEX Upgrade, this has been proposed as an explanation for the faster stabilization of the DTM, when using additional electron heating in otherwise neutral beam heated discharges (Günter et al 2000a), which is observed to increase the pressure gradient and hence the bootstrap current in the negative magnetic shear region.

The use of plasma rotation for stabilizing double tearing modes seems, as in the case of ideal modes, not a viable solution for a burning fusion plasma. However, $q$ profiles with a large distance between the corresponding rational $q$ surfaces may inhibit DTMs. Of course the DTM can be avoided by excluding negative magnetic shear altogether, which seems difficult to be reconciled with the requirement of a large bootstrap current fraction. Besides, to also prevent the occurrence of NTMs, $q$ has to stay at least above $3 / 2$, which is expensive with regard to the required non-inductive current drive.

4.3.3. Resistive interchange modes. The existence of resistive interchange modes has already been predicted by theory some time ago (Glasser et al 1976), but they were observed for the first time only recently (Chu et al 1996, Ozeki et al 1998, In et al 2000). Resistive interchange modes become unstable either by combining sufficiently high $\beta_{\mathrm{N}}$ with a sufficiently large degree of magnetic shear reversal $\left(q_{0}-q_{\text {min }}\right)$ or at low $\beta_{\mathrm{N}}$, if the pressure gradient is positive, i.e. the pressure profile is hollow. The latter, however, is rarely the case in tokamak plasmas. It is worth noting, that violating the Mercier criterion, which describes the ideal interchange stability limit, includes the destabilization of resistive interchange modes (Glasser et al 1976). 
In other words, the stability of the resistive interchange mode is a sufficient condition for the stability of the corresponding ideal mode.

As discussed above, resistive interchange modes destabilized by large values of $\beta_{\mathrm{N}}$ and $\left(q_{0}-q_{\min }\right)$ have been proposed in DIII-D as an alternative to the $\beta$-limit by ideal kink modes (Chu et al 1996, Lao et al 1996a, Strait et al 1997). The resistive interchange modes give rise to the local relaxation of pressure, rotation and $q$ profiles (Chu et al 1996), but can also cause disruptions by coupling to other modes such as DTMs and, thus, generating more global MHD instability. Alcator C-Mod discharges with slightly hollow pressure profiles $(\mathrm{d} p / \mathrm{d} r>0)$ and reversed magnetic shear, on the other hand, seem to confirm the second possibility of destabilizing interchange modes at low $\beta_{\mathrm{N}}$ (In et al 2000).

\section{Summary and conclusions}

In magnetic confinement fusion research, the tokamak has developed to a stage where it is considered a viable concept for a future burning plasma experiment and thereafter possibly for an electricity producing power plant. The reference confinement scenario, used for extrapolation to a burning fusion plasma, is based on the H-mode, which exhibits a transport barrier at the plasma edge. Ongoing research in plasmas with ITBs is generally motivated by the aims, to achieve (i) confinement regimes exceeding the reference scenarios with respect to confinement and stability, $\beta_{\mathrm{N}} H / q_{95}^{2}$, and (ii) a high bootstrap current fraction for a large proportion of non-inductive current drive.

Often, confinement modes with internal transport barriers are also referred to as advanced tokamak scenarios, which are basically defined by the same aims, namely improving confinement, stability and bootstrap current fraction. Therefore, to distinguish between ITBs and advanced tokamak scenarios in general, in this paper possible definitions of ITBs have been presented, which classify ITB discharges as a subgroup of advanced tokamak scenarios. The advanced tokamak scenarios comprise a much wider range of operating regimes, which do not necessarily need ITBs to attain the goals described above.

Looking at the large variety of names, used in the literature for similar or different confinement regimes, possible definitions of ITBs are discussed in order to have some kind of classification to distinguish ITBs from other types of confinement modes. The name "internal transport barrier" suggests a local reduction of the heat conductivity, which is generally governed by anomalous transport. Anomalous transport, driven by turbulence, usually raises the heat conduction or particle diffusion above the minimum level of the neoclassical transport generated by binary collisions.

However, defining an ITB as a region inside the plasma, where the transport drops to neoclassical levels has drawbacks. First, it is not straightforward to predict the neoclassical ion transport close to the plasma centre, due to finite orbit effects especially at large $q_{0}$ and orbit squeezing effects. Second, experimentally, even for very good electron confinement the neoclassical electron heat conduction, which is much lower than the corresponding ion heat conduction, is not reached. Third, in the presence of stiff temperature profiles, the heat conductivity is not a genuine plasma property, but is merely a consequence of the plasma edge conditions and the heat fluxes applied to the plasma.

Stiff temperature profiles are characterized by constant gradient lengths in the core confinement region of the plasma. In accordance with turbulence theories, which attribute the radial transport to temperature gradients and trapped particle modes, there is increasing experimental evidence that, beyond a certain critical gradient $R / L_{T}$, both ion and electron temperature profiles show some degree of profile stiffness, provided the heat flux in the 
respective transport channel is sufficiently high and the profiles are not determined by MHD instabilities.

In the presence of critical gradient limited or stiff temperature profiles any temperature increase in the plasma core at a given $R / L_{T}$ can only be achieved by raising the edge temperature. This is attained by decreasing the edge density at a given pedestal pressure or increasing the pedestal pressure, as observed for edge confinement modifications such as the L- to H-mode transition. An additional implication of stiff temperature profiles is that, at given $R / L_{T}$ and pedestal pressure an increase of the plasma energy is obtained only if the internal density gradient increases. However, as within certain limits $R / L_{T}$ itself depends on a number of plasma parameters, e.g. density gradient or ratio of electron to ion temperature, the internal plasma confinement can also change due to variations of the ambient discharge conditions.

\subsection{ITB definitions}

Assuming stiff temperature profiles in L- and H-mode plasmas, the first definition of ITBs is based on deviations from this profile stiffness, i.e. $R / L_{T}>R /\left.L_{T}\right|_{\text {crit }}$. The main quantities which determine such a confinement transition are magnetic shear and $E \times B$ shear flows. Here, it is important to distinguish, on the one hand, between the long to medium wavelength ITG and TEM, potentially determining ion heat, electron heat and particle transport, and, on the other hand, the short wavelength ETG modes, which only play a role in the electron heat transport. While the turbulence of ITG and TEMs should be reduced or suppressed by sheared $E \times B$ flows, they are considered ineffective on the small spatial scales of the ETG modes. The main stabilization mechanisms of ETG modes include low or negative magnetic shear and $\alpha$ stabilization, owing to increases of the Shafranov shift. The reduction of plasma turbulence as the main cause for the formation of ITBs is confirmed at least for parts of the turbulent spectrum by measurements of the plasma fluctuations (Fonck et al 1990, Sattler et al 1993, Nazikian and Mazzucato 1995, Mazzucato et al 1996, Nazikian et al 1998, McKee et al 1999, 2000b, Conway et al 2000, 2001).

An alternative definition of ITBs, namely $\rho_{\mathrm{T}}^{*}=\rho_{\mathrm{S}} / L_{\mathrm{T}}>\rho_{\mathrm{ITB}}^{*}$, derives from the requirement $\omega_{E \times B}>\gamma_{\max }^{\text {lin }}$ to stabilize ITG or trapped electron modes. It also contains the temperature gradient length as one of the crucial parameters, but in addition, the toroidal magnetic field and a $\sqrt{T}$ term from the normalization to $\rho_{\mathrm{s}}$. Although based on simplifications, e.g. effectively not considering the turbulence stabilization by toroidal rotational shear (Garbet 2002), this ITB definition successfully describes the toroidal magnetic field dependence of the ITB power threshold in JET positive magnetic shear (or OS) plasmas. Therefore, it is now routinely used to identify ITBs in JET.

However, the two ITB definitions presented here are not consistent in all cases, since temperature profiles with the same $R / L_{T}$ can have different $\rho_{\mathrm{T}}^{*}$. This and other shortcomings, such as not considering density profiles and the possibility that critical gradients may still exist in the presence of ITBs, certainly require further refinements.

\subsection{Experiments}

Generally, ITBs in tokamaks are produced by modifications of the current profile. The most common technique to generate low or negative central magnetic shear is to apply auxiliary heating during the initial current ramp-up phase of the tokamak discharge and thus to some extent "freeze" the initial skin current profile. Depending on the different heating methods and their different ion and electron heating partition, ITBs of the ions, electrons or both 
are generated with either $T_{\mathrm{i}}>T_{\mathrm{e}}, T_{\mathrm{i}}<T_{\mathrm{e}}$, or $T_{\mathrm{i}} \approx T_{\mathrm{e}}$. Usually, within the uncertainties of measurements and theoretical predictions, the ion heat transport becomes neoclassical at the ITB location. In discharges with predominantly ion heating this results in formerly inaccessible central ion temperatures of $T_{\mathrm{i}} \approx 15-25 \mathrm{keV}$ for medium size tokamaks, such as ASDEX Upgrade and DIII-D, and $T_{\mathrm{i}}>30 \mathrm{keV}$ for large tokamaks, such as JT-60U and JET. Correspondingly, $Q_{\mathrm{DT}}^{\mathrm{eq}}$ between, for instance, 0.3 in DIII-D and 1.2 in JT-60U have been achieved transiently. In TFTR (Bell et al 1997) and JET (Gormezano et al 1998) ITBs have been produced using the relevant fuel mixture of deuterium and tritium for a burning fusion plasma, resulting in actual $Q_{\mathrm{DT}}$ values of 0.4 in JET (Söldner et al 1999).

With the exception of the PEP mode, the density in ITB discharges is too low for a strong collisional coupling of ions and electrons. Hence, also $T_{\mathrm{i}} \approx T_{\mathrm{e}}$ is only attained if the heat fluxes into both plasma species are similar. To achieve sufficient ion heating and a high fusion reaction rate in a plasma dominated by $\alpha$-particle heating, future research will have to attempt raising the density in ITB plasmas. In comparison to the ITER-FEAT reference scenario, which is the high triangularity, high density $\mathrm{H}$-mode with $\bar{n}_{\mathrm{e}} / n_{\mathrm{GW}} \approx 0.8-0.9$, most present day ITB discharges operate at much lower densities. Also, efficient divertor pumping, in particular, requires higher edge densities.

The basic reason for operating at low density in present-day ITB plasmas is that the generation and sustainment of low or reversed magnetic shear $q$ profiles require high $T_{\mathrm{e}}$ values in order to achieve a low current diffusion or skin time. This means that for a given heating power the density has to be kept low to maximize $T_{\mathrm{e}}$. In first experiments aimed to raise the density without changing the $q$ profile, the density and heating power during the current ramp have been increased simultaneously in order to leave the electrical resistivity of the plasma unchanged (Lomas et al 2001).

The density also plays an important role with respect to the steady state sustainment of the ITB. The efficiency of external current drive methods (driven current per applied power) generally decreases with increasing density. However, to obtain a high bootstrap current fraction, the density gradient is more efficient than the corresponding temperature gradient, which favours a stronger density profile peaking. A further aspect, associated with increasing the density in NBI heated plasmas, is the dependence of the NBI power deposition on the density profile. As an increase of the density in the outer parts of the plasma moves the power deposition from the centre to larger radii, this may adversely affect the ITB which, for its sustainment, requires a certain heat flux inside the ITB region.

It is not yet clear whether transport effects exist which prevent an increase of density or density gradients above the values achieved in present experiments. On the one hand, density gradients may stabilize pure ITG modes. On the other hand, the turbulence drive of combined ITG and TEMs may increase with increasing density gradients. Besides, a density increase leads to a convergence of $T_{\mathrm{i}}$ and $T_{\mathrm{e}}$ which, depending on the starting point, $T_{\mathrm{i}}>T_{\mathrm{e}}$ or $T_{\mathrm{i}}<T_{\mathrm{e}}$, can be destabilizing for both ITG and ETG modes. However, if one argues that in ITB plasmas at least some of those modes are completely suppressed by magnetic or $E \times B$ flow shear, such a density dependence of the transport may not occur at all, which for a burning fusion plasma would favour ITBs over confinement modes which are still dominated by anomalous transport.

As yet, experiments with simultaneous electron and ion heating have not given a conclusive answer on the effect of $T_{\mathrm{e}}$ approaching $T_{\mathrm{i}}$ in ITB plasmas. They have shown both a degradation of confinement when applying additional electron heating into plasmas with originally $T_{\mathrm{i}}>T_{\mathrm{e}}$, and the build-up of strong electron ITBs without an adverse effect on the ion heat transport. In the latter case, explanations for the co-existence of electron and ion ITBs include $\alpha$-stabilization and the increase of the $E \times B$ shearing rate by local modifications of $B_{\theta}$. The PEP mode 
shows that, in principle, under certain conditions, it is possible to produce an ITB even with an equipartition between the ion and electron energy.

The formation of ITBs is often described as a bifurcation process requiring a certain threshold power similar to the H-mode, however, with a more complicated dependence on plasma parameters. The transition from an L- or H-mode regime to an ITB is associated with the stabilization of the turbulence in particular by low or negative magnetic shear and sheared $E \times B$ flows. Thereby, many possibilities exist to raise $\omega_{E \times B}$ which depends on the radial derivatives of the radial electric field and the poloidal magnetic field. In plasmas with unidirectional NBI, the sheared toroidal plasma rotation is considered to dominate $\omega_{E \times B}$, while with balanced NBI this role is taken up by the pressure gradient and poloidal rotation terms in $E_{r}$. But even with balanced NBI, although the net toroidal rotation is close to zero, the radial distribution of $v_{\phi}$ can exhibit rather strong gradients, which contribute significantly to $\omega_{E \times B}$.

Experimentally, the lowest ITB power threshold is observed for NBI parallel to the plasma current and negative magnetic shear. The power threshold increases for balanced NBI or injection opposite to the plasma current. Whether and how much angular momentum is required to form or sustain an ITB is a critical issue for the portability/applicability of ITB discharges to a burning plasma experiment, which still needs to be answered. Since, in the absence of angular momentum, $\omega_{E \times B} / \gamma_{\max }^{\operatorname{lin}}$ essentially scales like $\rho_{T}^{*}$, and $\rho_{T}^{*}$ will be smaller at the higher magnetic field and larger major radius of a future fusion device, the confinement improvement will have to rely more on magnetic shear stabilization of the turbulence.

Experimental results suggest that in many cases, rather than having a continuous increase of the $E \times B$ shearing rate until the ITB transition occurs, when $\omega_{E \times B}$ exceeds $\gamma_{\max }^{\text {lin }}$, discrete trigger events seem to cause the transition to improved confinement. They include large local excursions of the poloidal rotation velocity prior to the ITB formation, a correlation between ITB formation and the occurrence of low order rational $q$ surfaces in the plasma edge and core and a stepwise growth and expansion of the ITB. Provided the applied heat flux inside the ITB is sufficiently large, experiment and theory suggest that the ITB expands until $\omega_{E \times B}$ is balanced by $\gamma_{\max }^{\text {lin }}$.

Regarding the ITB formation, there is clear experimental evidence that MHD instabilities associated with low order rational values of $q$ can act as a trigger by locally increasing $\omega_{E \times B}$. Also the rarefaction of low order rational $q$ surfaces near the minimum of the $q$ profile has been proposed as a possible ITB trigger mechanism. During the further evolution of the ITB, the role of rational $q$ surfaces is less clear. While some experiments do not find a connection between certain points in the $q$ profile and the ITB location, others suggest that the ITB location either follows rational $q$ surfaces or the minimum of the $q$ profile for reversed magnetic shear configurations. However, in the latter case this does not have to be a genuine transport effect, since a large bootstrap current fraction also supports a correlation between $\rho_{\text {ITB }}$ and $\rho_{q_{\min }}$.

\subsection{Steady state}

For exploiting the potential steady state properties of ITB discharges it is necessary to avoid impurity accumulation, guarantee sufficient fast particle confinement and avoid detrimental MHD instabilities by possibly making use of local MHD oscillations for pressure and particle control.

The concentration of impurities has to be low enough to neither unduly dilute the fuel ions nor radiate too much energy from the plasma core. As energy and particle transport are closely related, there is a concern that, by reducing the heat transport in ITB plasmas, 
the particle and, in particular, the impurity concentrations may rise to intolerable levels. As predicted by neoclassical theory, the inward convection caused by strong density gradients frequently results in the accumulation of impurities which worsens with increasing impurity charge, $Z$. To avoid this process a compromise has to be found between the good confinement properties, in particular, of reversed magnetic shear configurations, which are the reason for the high bootstrap current fractions, and the need for low impurity concentrations. This requires a limitation of the density profile peaking and a close alignment of density and temperature gradients, to make the best use of the temperature screening of impurities.

A further issue is fast particle confinement. Heating of the plasma by the $3.5 \mathrm{MeV}$ $\alpha$-particles from the $\mathrm{D}-\mathrm{T}$ fusion reaction requires that they are confined inside the burning zone in the core of the plasma. However, since the size of the trapped particle orbits depends on the strength of the plasma current and the poloidal magnetic field distribution, the confinement of fast particles has to be carefully considered when extrapolating low or negative magnetic shear configurations to a burning fusion plasma (Tobita et al 1997,Zweben et al 1998, Kusama et al 1999, Ruskov et al 1999, Stratton et al 1999). It is worth noting that, in contrast to ICRF heated ${ }^{4} \mathrm{He}$ ions (Mantsinen et al 2002), the velocity distribution of fast $\alpha$-particles from the fusion reaction is isotropic. Thus, due to a lower trapped particle fraction, the overall $\alpha$-particle confinement is better than that of the ICRF ions.

The differences between the orbits of $1 \mathrm{MeV}$ tritons in a normal, positive magnetic shear and a reversed magnetic shear configuration are illustrated in figure 52. Clearly, the negative magnetic shear in the plasma centre increases the orbit sizes. However, not only first orbit losses but also a displacement of the $\alpha$-particles outside the ITB have to be avoided, since this will not allow a sustained fusion reaction and, in the absence of another central heat source, will result in the collapse of the ITB. Possible solutions to this problem include the presence of a sufficiently high plasma current and the limitation of the magnetic shear reversal. However, the necessary increase of the plasma size in a future fusion device, in order to achieve burning plasmas, helps to confine the fast particles.

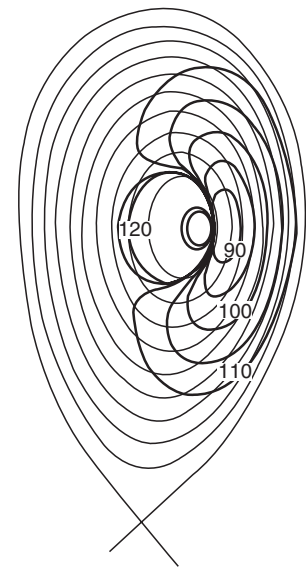

Normal shear

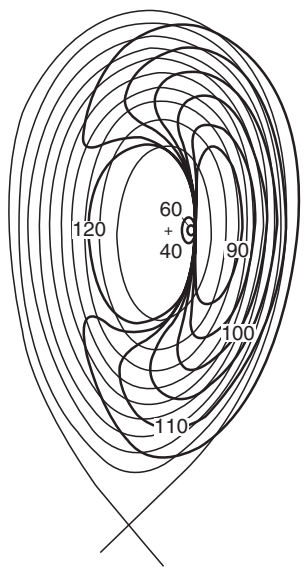

Reversed shear

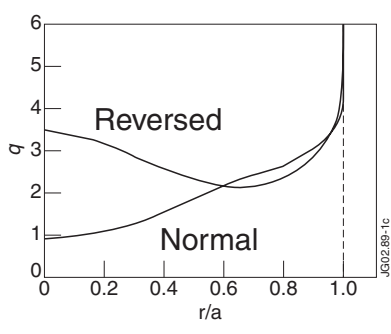

Figure 52. Calculated guiding centre orbits in JT-60U of a $1 \mathrm{MeV}$ triton launched at $r / a=0.2$, comparing normal (positive) and negative magnetic shear configurations (reproduced from (Tobita et al 1997); copyright in these materials is vested in the International Atomic Energy Agency, Vienna, Austria from which permission for publication must be obtained). The plasma current is the same in both cases. The numbers indicate the pitch angles of the tritons in degree. 
In addition to providing improved confinement, the current profiles used to produce ITBs have further advantages. They avoid low order rational $q$ values and the associated MHD instabilities, such as sawtooth oscillations or neoclassical tearing modes, and, owing to the low magnetic shear, open access to the second stability regime for high- $n$ ballooning modes. Consequently, the pressure gradients can reach values which are not attainable with conventional current profiles.

However, the control of MHD instabilities driven by these pressure gradients, coinciding with low magnetic shear, remains a challenge. Such MHD instabilities include low- $n$ ideal kink-ballooning type (infernal) modes or, for reversed magnetic shear configurations, also resistive interchange and double tearing modes. Generally, broader pressure profiles, i.e. lower pressure profile peaking, increases the $\beta$ limit, which means that the low magnetic shear region has to be moved to larger radii and the pressure gradient at the ITB has to be reduced. Also, a clear spatial separation of ITB location and low magnetic shear region improves the stability properties. Up to now, $\beta_{\mathrm{N}}$ values considerably above two could be reached only transiently in ITB discharges and then often combined with a higher $q_{95}$ (ITER Physics Expert Groups 1999), which despite the high $\mathrm{H}$-factors does not necessarily increase the figure of merit $\beta_{\mathrm{N}} H / q_{95}^{2}$. A significant increase of $\beta_{\mathrm{N}}$ may require a conducting wall close to the plasma edge and saddle coils to stabilize the radial displacements caused by the MHD instabilities.

As has been recognized in the H-mode, MHD instabilities in the form of relaxation oscillations, such as ELMs, can also become a useful tool for pressure and particle control, provided they are only local perturbations of the equilibrium. In the core of ITB plasmas such MHD modes would be desirable to limit the pressure gradients. In some cases such local modes have already been observed, however, depending on the details of the $q$ profile, they often change into global modes destroying the confinement or even leading to the termination of the discharge.

ELMs are relaxation oscillations at the plasma edge which in principle exhibit the desired pressure and particle control properties. With regard to the H-mode, the problem is that for Type-I ELMs the divertor target load resulting from the strong heat pulses may be too large when extrapolating to a future fusion device. Combined with ITBs, strong ELMs have a second undesired effect. In particular, for plasmas with wide internal transport barriers, large ELMs tend to also perturb the ITB, leading to an erosion of the improved confinement zone. As pointed out above, achieving a broad improved confinement region is not only beneficial for maximizing the energy content of the plasma, but also improves the stability properties. Recent results which show plasma edge regimes with a more continuous heat flow, for instance, in the form of small ELMs, are encouraging for both the reduction of the peak target load and the combination of edge and internal transport barriers.

Confinement and stability are closely linked to the toroidal current density distribution. Hence, in particular, in plasmas with large bootstrap current fractions, the bootstrap current profile has to be consistent with confinement and stability requirements of the total current profile. Due to the limited efficiency of external current drive methods, a large bootstrap current fraction is necessary to minimize the inductive (Ohmic) current. This favours plasmas with ITBs as they exhibit a rather narrow bootstrap current profile at the barrier location which naturally supports the low or negative magnetic shear usually required to generate the ITB.

Recently it has been shown that full non-inductive current drive with considerable fractions, $\geqslant 50 \%$, of the bootstrap current generated by an ITB is possible, at least at reduced plasma current and $\beta$. In figure 53 this is exemplified for a JET discharge, in which the near steady state conditions are achieved by the feedback control of the neutron rate and the local ITB strength. The bootstrap current is supplemented by LHCD which also serves to avoid 


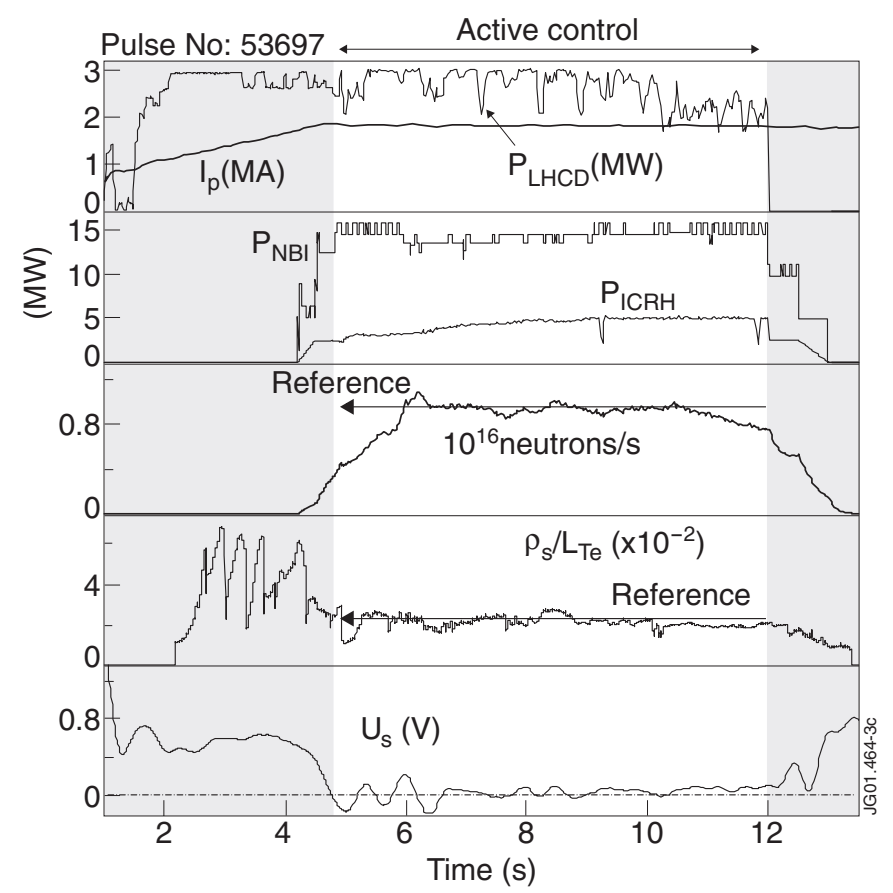

Figure 53. Time evolution of a JET discharge (1.8 MA, 3.4 T) with an internal transport barrier and full non-inductive current drive (surface loop voltage $U_{\mathrm{s}}=0$ ), using feedback (active) control of the neutron rate by NBI and the local ITB strength, $\rho_{\mathrm{T}}^{*}=\rho_{\mathrm{s}} / L_{T_{\mathrm{e}}}$, by ICRH (reproduced from (Mazon et al 2002)). LHCD not only serves as a method of external current drive but also suppresses strong ELM activity.

strong ELM activity. However, extrapolating this scenario to a burning fusion plasma implies the non-trivial assumption that the bootstrap current fraction can be further increased. Another aspect which needs to be considered in a plasma with strong $\alpha$-particle heating is the effect of the fast particle population on the bootstrap current distribution.

The research into the physics of ITBs in tokamak plasmas has contributed notably to the understanding of plasma transport and stability. Although for future applications of ITB plasmas a number of problems still have to be solved, it is in particular the potential steady state capability which makes it attractive to pursue the development of ITB plasmas as one of several options for an advanced tokamak scenario.

\section{Acknowledgments}

I would like to thank, in particular, Friedrich Wagner and Michael Kaugmann for the opportunity to carry out this work and their encouragement. Moreover, I am grateful to the Max-Planck-Institut für Plasmaphysik in Garching for giving me the time for such an extensive study. In addition, I would like to acknowledge the many helpful and fruitful discussions with my colleagues at IPP Garching and JET. In particular, I would like to thank Xavier Garbet, Otto Gruber and George Sips for reading and correcting the final manuscript. Finally, I would like to thank Raymond Koch and the science faculty of the University of Mons-Hainaut, Belgium for the opportunity to submit this work for the degree d'Agrégé de l'Enseignement Supérieur. 


\section{Appendix}

Appendix A.1. Tokamak experiments

Table A1.

\begin{tabular}{|c|c|c|c|c|c|c|}
\hline Device (Institution) & $R(\mathrm{~m})$ & $a(\mathrm{~m})$ & $\kappa$ & $B_{\phi}(\mathrm{T})$ & $I_{\mathrm{p}}(\mathrm{MA})$ & Configuration \\
\hline $\begin{array}{l}\text { Alcator C-Mod } \\
\text { (MIT, USA) }\end{array}$ & 0.67 & 0.22 & 1.7 & 8.1 & 1.5 & Divertor \\
\hline $\begin{array}{l}\text { ASDEX Upgrade } \\
\text { (IPP, Germany) }\end{array}$ & 1.65 & 0.50 & 1.6 & 3.9 & 1.2 & Divertor \\
\hline $\begin{array}{l}\text { COMPASS-D } \\
\text { (UKAEA, UK) }\end{array}$ & 0.56 & 0.23 & 1.7 & 2.1 & 0.3 & Divertor \\
\hline $\begin{array}{l}\text { DIII-D } \\
\text { (GA, USA) }\end{array}$ & 1.67 & 0.67 & $\leqslant 2$ & 2.1 & 1.6 & Divertor \\
\hline $\begin{array}{l}\text { FT-U } \\
\qquad \text { (ENEA, Italy) }\end{array}$ & 0.93 & 0.30 & 1.0 & 8.0 & 1.3 & Limiter \\
\hline $\begin{array}{l}\text { JET } \\
\text { (Euratom, EU) }\end{array}$ & 3.1 & 1.25 & 1.6 & 4.0 & $5.0(7.0)$ & Divertor \\
\hline $\begin{array}{l}\text { JT-60U } \\
\text { (JAERI, Japan) }\end{array}$ & 3.4 & 1.1 & $<1.8$ & 4.2 & 2.5 & Divertor \\
\hline $\begin{array}{l}\text { RTP } \\
\text { (FOM, The Netherlands) }\end{array}$ & 0.72 & 0.16 & 1 & 2.4 & 0.15 & Limiter \\
\hline $\begin{array}{l}\text { TEXTOR } \\
\text { (FZJ, Germany) }\end{array}$ & 1.75 & 0.46 & 1 & 3.0 & 0.6 & Limiter \\
\hline $\begin{array}{l}\text { T-3 } \\
\text { (Kurchatov, Russia) }\end{array}$ & 1.0 & 0.15 & 1 & 3.5 & 0.12 & Limiter \\
\hline $\begin{array}{l}\text { T-10 } \\
\text { (Kurchatov, Russia) }\end{array}$ & 1.5 & 0.37 & 1 & 4.5 & 0.7 & Limiter \\
\hline $\begin{array}{l}\text { TCV } \\
\text { (CRPP, Switzerland) }\end{array}$ & 0.88 & 0.24 & 2.6 & 1.4 & 1.0 & Limiter \\
\hline $\begin{array}{l}\text { TFTR } \\
\text { (PPPL, USA) }\end{array}$ & 2.4 & 0.80 & 1 & 5.0 & 2.2 & Limiter \\
\hline $\begin{array}{l}\text { Tore Supra } \\
\qquad \text { (CEA, France) }\end{array}$ & 2.37 & 0.80 & 1 & 4.5 & 2.0 & Limiter \\
\hline
\end{tabular}

\section{Appendix A.2. Estimate for $Q_{\mathrm{DT}}(R)$}

Here, an estimate is given for the dependence of the power multiplication factor $Q_{\mathrm{DT}}=$ $P_{\text {fusion }} / P_{\mathrm{h}}$ on the major radius $R$ of the toroidal plasma, in order to demonstrate the effect of the tokamak size on the realization of a burning fusion plasma.

The power balance of the fusion reaction is given by

$$
P_{\mathrm{h}}+P_{\alpha}=\frac{W}{\tau_{\mathrm{E}}}
$$

where $P_{\mathrm{h}}$ is the external heating power supplied, $P_{\alpha}$ the power carried by the $\alpha$-particles of the fusion reaction, and $W / \tau_{\mathrm{E}}$ the rate of energy loss from the plasma. The neutrons produced in the fusion reaction are not confined and hence do not contribute to the plasma power balance. Since four times more energy of the deuterium-tritium fusion reaction is going into the neutrons 
than into the $\alpha$-particles, $P_{\text {fusion }}=5 P_{\alpha}$ and hence

$$
Q_{\mathrm{DT}}=\frac{5 P_{\alpha}}{P_{\mathrm{h}}} .
$$

Approximating the plasma energy $W$ by

$$
W=3 \overline{n T} V
$$

which assumes $T=T_{\mathrm{e}}=T_{\mathrm{i}}$ and $n=n_{\mathrm{e}}=n_{\mathrm{D}}+n_{\mathrm{T}}$, and where $V=\pi a b 2 \pi R$ is the plasma volume in the form of a torus with elliptical cross section ( $a$ and $b$ are the horizontal and vertical minor radii, respectively). $\overline{n T}=\int n T \mathrm{~d} V / \int \mathrm{d} V$ denotes the volume average of the product of density and temperature profiles. Similarly,

$$
P_{\alpha}=\frac{1}{4} \bar{n}^{2}\langle\sigma v\rangle \varepsilon_{\alpha} V
$$

$\langle\sigma v\rangle$ is the fusion reaction rate and $\varepsilon_{\alpha}=3.5 \mathrm{MeV}$ the energy per $\alpha$-particle. For the empirical $\tau_{\mathrm{E}}$-scaling an approximate form is used in order to finally obtain an analytical expression for $Q_{\mathrm{DT}}(R)$

$$
\tau_{\mathrm{E}}=H f\left(B_{\mathrm{T}}, M, \kappa, \varepsilon, \overline{n_{\mathrm{e}}}\right) \frac{I R^{2}}{\sqrt{P}},
$$

where $H$ is the confinement enhancement factor. Depending on whether an L- or H-mode scaling is chosen, $H \approx 1$ or 2 respectively. The actual exponents for $R$ and the plasma current $I$ differ slightly from the ones in equation (A5). For typical scaling laws the difference is $10 \%$ or less. The degradation of the confinement with total heating power $P=P_{\mathrm{h}}+P_{\alpha}$ is equal or somewhat stronger than $\sqrt{P}$ (Yushmanov et al 1990, H-mode Database Working Group 1994, ITER Confinement Database and Modelling Working Group 1997). The other scaling parameters are contained in $f$ (line averaged density $\overline{n_{\mathrm{e}}}$, elongation of the plasma cross section $\kappa$, atomic mass $M$, inverse aspect ratio $\varepsilon=a / R$ and toroidal magnetic field $B_{\mathrm{T}}$ ). In the ion temperature range of $10-20 \mathrm{keV}$ the fusion reaction rate is approximately given by

$$
\langle\sigma v\rangle=c_{\sigma v} T^{2}
$$

where $c_{\sigma v}=1.1 \times 10^{-24} \mathrm{keV}^{-2} \mathrm{~m}^{3} \mathrm{~s}^{-1}$. Combining equations (A1)-(A6) yields

$$
Q_{\mathrm{DT}}=5\left(\frac{24 \pi^{2}}{\varepsilon_{\alpha} c_{\sigma v}} \frac{\overline{n T}}{\overline{n^{2} T^{2}}} \frac{a b}{f^{2} H^{2} I^{2} R^{3}}-1\right)^{-1} .
$$

The second term in the denominator $c_{n T}=\overline{n T}^{2} / \overline{n^{2} T^{2}}$ is a constant depending on the shape of the density and temperature profiles. It turns out that in $c_{n T}$ temperature and density profiles cancel exactly only if $\tau_{\mathrm{E}} \sim \sqrt{P}$. In the following, it is assumed that elongation $\kappa=b / a$ and inverse aspect ratio are constants. This permits replacement of $a b$ by $\kappa \varepsilon^{2} R^{2}$. Thus

$$
Q_{\mathrm{DT}}=5\left(\frac{24 \pi^{2}}{\varepsilon_{\alpha} c_{\sigma v}} \frac{c_{n T} \kappa \varepsilon^{2}}{f^{2} H^{2} I^{2} R}-1\right)^{-1} .
$$

Replacing the plasma current by the edge $q$ or for a divertor configuration by $q_{95}$,

$$
q=g_{\mathrm{s}} \frac{a b B_{\mathrm{T}}}{R I}
$$

results in

$$
Q_{\mathrm{DT}}=5\left(\frac{24 \pi^{2}}{\varepsilon_{\alpha} c_{\sigma v}} \frac{c_{n T} q^{2}}{\kappa \varepsilon^{2} g_{s}^{2}} \frac{1}{f^{2} H^{2} B_{\mathrm{T}}^{2} R^{3}}-1\right)^{-1} .
$$

$g_{\mathrm{s}}$ is a parameter which depends on aspect ratio and plasma shape. As $M$ does not change and $\kappa$ and $\varepsilon$ are kept constant, the two quantities left in $f$ to be considered are the line averaged 
density and toroidal magnetic field. Depending on which scaling is chosen, the exponent of $\overline{n_{\mathrm{e}}}$ varies between 0.1 and 0.4 . The latter would already imply an almost linear dependence on $\overline{n_{\mathrm{e}}}$ in the denominator of equation (A10). A possible treatment would be to express $\overline{n_{\mathrm{e}}}$ in terms of the Greenwald density $n_{\mathrm{G}}=I / \pi a^{2}=I / \pi \varepsilon^{2} R^{2}$ (Greenwald et al 1988), keeping the Greenwald fraction $\overline{n_{\mathrm{e}}} / n_{\mathrm{G}}$ constant, which would add another plasma current and major radius dependence. In the extreme case, this would reduce the major radius dependence in equation (A10) from $R^{3}$ to $R^{2}$. The toroidal magnetic field scalings range from $B_{\mathrm{T}}^{0.03}$ to $B_{\mathrm{T}}^{0.45}$ which would increase the exponent of $B_{\mathrm{T}}$ up to 3 . For simplicity, however, the $\overline{n_{\mathrm{e}}}$ and $B_{\mathrm{T}}$ dependences in $f$ are neglected.

To determine the constants in equation (A10), the ITER-FDR design parameters for an ignited plasma ( $Q_{\mathrm{DT}} \rightarrow \infty$ ) are used (ITER Physics Expert Groups 1999). The other points, entered in figure 1, are taken from an ASDEX Upgrade steady state high performance plasma, from the JET deuterium tritium experiment (stationary ELMy H-mode discharge) (Gibson et al 1998 ) and the ITER-FEAT design report. The JET point agrees with the value for $Q_{\mathrm{DT}}$ from the analytical expression within a factor of three, not taking into account that, with central values of $T_{\mathrm{i}}=T_{\mathrm{e}}=8 \mathrm{keV}$, the JET plasma already lies slightly below the assumed temperature range of 10-20 keV. The parameters of the ASDEX Upgrade discharge, in addition, violate the assumption of $T_{\mathrm{i}}=T_{\mathrm{e}}$. The inclusion of $\overline{n_{\mathrm{e}}}$ from the scaling expression $f$ would weaken the $R$-dependence and hence reduce this discrepancy. Nevertheless, the JET and ASDEX Upgrade data show that the $Q_{\mathrm{DT}}(R)$ given reproduces the general trend quite well.

\section{Appendix A.3. Radial electric field}

In a tokamak equilibrium the electric field consists of components in the magnetic flux surface and a radial component which is perpendicular to the magnetic surface (Wesson 1997). While discharges are formed by the application of a toroidal electric field, the radial electric field $E_{r}$ is part of the radial force balance, which must be satisfied for each of the plasma species (electrons and different plasma ions). Denoting the plasma species by $j$, it can be written as

$$
n_{j} e_{j}\left(E_{r}+(\vec{v} \times \vec{B})_{r}\right)=\frac{\mathrm{d} p_{j}}{\mathrm{~d} r},
$$

where $e_{j}=Z_{i} e$ for ions and $e_{j}=-e$ for electrons. Summing over all species (electrons and ions) and considering the quasi-neutrality condition

$$
n_{\mathrm{e}}=\sum Z_{i} n_{i}
$$

the basic equilibrium equation

$$
\vec{j} \times \vec{B}=\nabla p
$$

is obtained, which describes that the magnetic forces $\vec{j} \times \vec{B}$, where $\vec{j}=-n_{\mathrm{e}} e \vec{v}_{\mathrm{e}}+\sum Z_{i} n_{i} \vec{v}_{i}$ is the current density, have to balance the pressure gradient $\nabla p$, to be in equilibrium. From equation (A13) the Grad-Shafranov equation is derived (Freidberg 1982), which is used to numerically calculate axisymmetric tokamak equilibria. In order to solve the GradShafranov equation, these equilibrium codes restrict the number of free parameters, which are constrained by various measurements, including the magnetic flux outside the plasma and more recently local internal magnetic field measurements by means of the Motional Stark Effect (MSE) (Levinton et al 1989, Wroblewski et al 1990, Rice et al 1995, Wolf et al 1997, Hawkes et al 1999).

The radial force balance, as given in equation (A11) is used to infer the radial electric field from measurements of the pressure gradient $\left(\mathrm{d} p_{x} / \mathrm{d} r\right)$ and the poloidal $\left(v_{\theta, x}\right)$ and toroidal $\left(v_{\phi, x}\right)$ rotation velocities of impurity ions by charge exchange recombination spectroscopy 
(CXRS) (Hellermann et al 1990, Hawkes et al 1994, Bell et al 1997, Bell et al 1999b,Meister et al 2001) and equilibrium reconstructions of the respective magnetic field components $B_{\theta}$ and $B_{\phi}$ :

$$
E_{r}=\frac{1}{Z_{x} e n_{x}} \frac{\mathrm{d} p_{x}}{\mathrm{~d} r}+v_{\phi, x} B_{\theta}-v_{\theta, x} B_{\phi} .
$$

The poloidal and toroidal impurity velocities are inferred from the Doppler shifted line emission of the ion species, produced by the charge exchange process between the neutral diagnostic or heating beam and the plasma ions. In the case of carbon as a fully ionized trace impurity in the plasma, the relevant ion species, the line emission of which is measured, is hydrogen-like carbon $\left(C^{5+}\right)$.

Recently, it has been discovered that the interpretation of the measurement of the poloidal rotation was incorrect. Besides the energy dependence of the charge exchange cross section (Hellermann and Summers 1992, Hellermann et al 1995), the ion gyration in combination with the finite life time of the excited state of the impurity ion leads to an apparent poloidal velocity (Bell and Synakowski 2000, Ernst et al 2000, Meister et al 2001), resulting in $v_{\theta, x} \propto T_{\mathrm{i}}$ (Bell et al 1999c), which is generally not consistent with neoclassical predictions. The correction of this effect in many cases is of the order of the apparent velocity itself and may even lead to a change of sign.

The temperatures and the toroidal rotation velocities of the different ion species are approximately the same $\left(v_{\phi, I} \approx v_{\phi, x}\right)$. Under these assumptions the radial force balance of the hydrogenic, main ion species $\left(Z_{\mathrm{I}}=1\right)$ becomes

$$
E_{r}=\frac{1}{e n_{\mathrm{I}}} \frac{\mathrm{d} p_{\mathrm{I}}}{\mathrm{d} r}-v_{\theta, \mathrm{I}} B_{\phi}+v_{\phi, x} B_{\theta} .
$$

For discharges heated by neutral beam injection, the profile shape of $v_{\phi, \mathrm{I}}$ is determined by the beam momentum deposition profile and the momentum diffusivity, and resembles that of the ion temperature (Ernst et al 1998b and references therein). In the absence of a poloidal rotation measurement equation (A15) can be used to derive the radial electric field, which, by substituting the poloidal rotation velocity of the main plasma ions by expressions derived from neoclassical theory (Ernst et al 1998b, Zhu et al 1999), is

$$
E_{r}=v_{\phi, x} B_{\theta}+F_{1} \frac{\mathrm{d} T_{I}}{\mathrm{~d} r}+F_{2} \frac{T_{I}}{n_{I}} \frac{\mathrm{d} n_{I}}{\mathrm{~d} r},
$$

where $F_{1}$ and $F_{2}$ are functions depending on the viscosity coefficients of the plasma and the impurity strength parameter $n_{x} Z_{x}^{2} / n_{\mathrm{I}} Z_{\mathrm{I}}^{2}$. To obtain equation (A16) with the functions $F_{1}$ and $F_{2}$, as given in (Ernst et al 1998b), low impurity densities are assumed (trace impurity limit). As $E_{r}$ is not a flux surface quantity, i.e., it can vary along a flux surface, the expressions for $E_{r}$ are usually given at the outer mid-plane, where, first, the measurements of temperatures, densities and velocities are usually made and, second, the microinstabilities due to their ballooning character exhibit the largest growth rates.

Another measurement of the radial electric field is obtained from extensions to the MSE diagnostic. Since the electric field, which is responsible for the Stark effect, not only contains the Lorentz electric field $E_{\mathrm{L}}$, caused by the motion of the neutral atoms with respect to the magnetic field of the tokamak, but also $E_{r}$, two independent measurements of $\vec{E}_{\mathrm{L}}+\vec{E}_{r}$ allow both fields to be separated (Rice et al 1997, Zarnstorff et al 1997, Levinton 1999, Rice et al $1999 b)$. As a general rule, $E_{r}$ becomes important, if $E_{r} / v_{\text {beam }} \approx B_{\theta}$, where $v_{\text {beam }}$ is the velocity of the neutral beam used for the MSE diagnostic.

Large excursions of the impurity poloidal rotation velocity have been observed in some tokamaks prior to the formation of strong ITBs (Bell et al 1998a, De Pena-Hempel et al 1998), 
for which the inferred $E_{r}$ change agrees well with MSE measurements (Levinton et al 1998). As the changes of $v_{\theta, x}$ exceed the corrections for the ion gyration and finite life time effects, agreement is observed even without considering them.

\section{References}

Alladio F et al 1999 Plasma Phys. Control. Fusion 41 A323

Anado A et al 1986 Phys. Rev. Lett. 562180

Antonsen T M et al 1996 Phys. Plasmas 32221

Baker D R et al 2001 Phys. Plasmas 84128

Baranov Y F et al 1999 Nucl. Fusion 391463

Barbato E 2001 Plasma Phys. Control. Fusion 43 A 287

Batha S H et al 1997 Phys. Plasmas 43614

Baylor L R et al 1997 Nucl. Fusion 37127

Becoulet A et al 2000 Nucl. Fusion 401113

Becoulet A et al 2001a Plasma Phys. Control. Fusion 43 A395

Becoulet $\mathrm{M}$ et al 2001b 28th Conf. on Controlled Fusion and Plasma Physics (Madeira, 2001) (EPS)

Becoulet M et al 2002 Plasma Phys. Control. Fusion 44 A103

Beer M A et al 1997 Phys. Plasmas 41792

Bell M G et al 1997 Phys. Plasmas 41714

Bell M G et al 1999a Plasma Phys. Control. Fusion 41 A 719

Bell R E et al 1996 Proc. 23rd Conf. on Controlled Fusion and Plasma Physics (Kiev, 1996) vol. 20C, part I (EPS) p. 59

Bell R E et al 1998a Phys. Rev. Lett. 811429

Bell R E et al 1999b Rev. Sci. Instrum. 70821

Bell R E et al 1999c Proc. 17th IAEA Fusion Energy Conf. (Yokohama, 1998) (IAEA)

Bell R E et al 1998b Plasma Phys. Control. Fusion 40609

Bell R E and Synakowski E J 2000 New understanding of poloidal rotation measurements. Proc. 12th APS Top. Conf. on Atomic Processes in Plasmas (Reno Nevada, March 2000) ed R C Mancini AIP

Bergmann A, Peeters A G and Pinches S D 2001 Phys. Plasmas 85192

Bernabei S et al 1982 Phys. Rev. Lett. 491255

Beurskens M N A et al 2001 Plasma Phys. Control. Fusion 4313

Bickerton R J, Connor J W and Taylor J B 1971 Nat. Phys. Sci. 239110

Biglari H, Diamond P H and Rosenbluth M N 1989 Phys. Fluids B 1109

Biglari H, Diamond P H and Terry P W 1990 Phys. Fluids B 21

Bondeson A et al 1999 Nucl. Fusion 391523

Bondeson A and Ward D J 1994 Phys. Rev. Lett. 722709

Boozer A H 1998 Phys. Plasmas 51647

Bracco G et al 2001 18th IAEA Conf. on Fusion Energy (Sorrento, 2000), IAEA-CN-77/EX6/3 (IAEA)

Brakel R et al 1997 Plasma Phys. Control. Fusion 39 B 273

Brunner S, Fivaz M, Tran T M and Vaclavik J 1998 Phys. Plasmas 53929

Budny R V et al 2002 Plasma Phys. Control. Fusion 441215

Buratti P et al 1997 Plasma Phys. Control. Fusion 39 B 383

Buratti P et al 1999 Phys. Rev. Lett. 82560

Burrell K H 1997 Phys. Plasmas 41499

Burrell K H et al 1991 13th IAEA Conf. on Plasma Phys. and Control. Nucl. Fusion Research (Washington, 1990) (IAEA)

Burrell K H et al 1992 Plasma Phys. Control. Fusion 341859

Burrell K H et al 2001 Phys. Plasmas 82153

Campbell D J 2001 Phys. Plasmas 82041

Carreras B A et al 1988 Nucl. Fusion 281613

Challis C D et al 1992 Nucl. Fusion 322217

Challis C D et al 2001a Plasma Phys. Control. Fusion 43861.

Challis C D et al 2001b 28th Conf. on Controlled Fusion and Plasma Physics (Madeira, 2001), P2.008 (EPS)

Challis C D et al 2002 Plasma Phys. Control. Fusion 441031

Chang C S and Hinton F L 1986 Phys. Fluids 293314

Chang Z et al 1995 Phys. Rev. Lett. 744663 
Chang Z et al 1996 Phys. Rev. Lett. 773553

Chen $\mathrm{H}$ et al 2001 Nucl. Fusion 4131

Chu M S et al 1996 Phys. Rev. Lett. 772710

Connor J W, Hastie R J, Wilson H R and Miller R L 1998 Phys. Plasmas 52687

Connor J W and Wilson H R 1994 Plasma Phys. Control. Fusion 36719

Conway G D et al 2000 Phys. Rev. Lett. 841463

Conway G D et al 2001 Plasma Phys. Control. Fusion 431239

Cottrell G A et al 1998 Plasma Phys. Control. Fusion 401251

Cottrell G A et al 1999 Nucl. Fusion 39389

Crisanti F et al 2002 Phys. Rev. Lett. 88145004

de Baar M R et al 1997 Phys. Rev. Lett. 784573

de Baar M R et al 1999 Phys. Plasmas 64645

De Pena-Hempel S et al 1998 Proc. 25th Eur. Conf. on Controlled Fusion and Plasma Physics (Prag 1998) (Geneva:

European Physical Society) 484

Dewar R L and Persson M 1993 Phys. Fluids B 54273

Diamond P H et al 1997 Phys. Rev. Lett. 781472

Dimits A M et al 2000 Phys. Plasmas 7969

Dominguez R R and Staebler G M 1993 Nucl. Fusion 3351

Doyle E J et al 2000 Plasma Phys. Control. Fusion 42 A 237

Doyle E J et al 2001 Plasma Phys. Control. Fusion 43 A 95

Doyle E J et al 2002 Nucl. Fusion 42333

Drake J F, Guzdar P N and Hassam A B 1988 Phys. Rev. Lett. 612205

Dux R et al 2001 28th Conf. on Controlled Fusion and Plasma Physics (Madeira, 2001), P2.007 (EPS)

Dux R et al 1999 Nucl. Fusion 391509

Efthimion P C et al 1998 Phys. Plasmas 51832

Efthimion P C et al 1999 Nucl. Fusion 391905

Ekedahl A et al 1998 Nucl. Fusion 381397

Equipe Tore Supra (presented by X Litaudon) 1996 Plasma Phys. Control. Fusion 38 A 251

Ernst D R, Coppi B, Scott S D, Porkolab M and TFTR Group 1998a Phys. Rev. Lett. 812454

Ernst D R et al 1998b Phys. Plasmas 5665

Ernst D R et al 2000 Phys. Plasmas 7615

Fiore C L et al 2001 Phys. Plasmas 82023

Fitzpatrick R and Jensen T H 1996 Phys. Plasmas 32641

Fonck R J, Duperrex P A and Paul S F 1990 Rev. Sci. Instrum. 613487

Forest C B et al 1994 Phys. Rev. Lett. 732444

Forest C B et al 1996 Phys. Rev. Lett. 773141

Freidberg J P 1982 Rev. Mod. Phys. 54801

Fröjdh M, Liljeström M and Nordman H 1992 Nucl. Fusion 32419

Fujita T et al 1997a Phys. Rev. Lett. 782377

Fujita T et al 1997b Plasma Phys. Control. Fusion 39 B 75

Fujita T et al 1998 Nucl. Fusion 38207

Fujita T et al 1999 Nucl. Fusion 391627

Fujita T et al 2001a Phys. Rev. Lett. 87245001

Fujita T et al 2001b Phys. Rev. Lett. 87085001

Fukada T et al 2001 28th Conf. on Controlled Fusion and Plasma Physics (Madeira, 2001), P4.004 (EPS)

Fukuyama A et al 1998 Plasma Phys. Control. Fusion 40653

Galeev A A and Sagdeev R Z 1968 Sov. Phys. JETP 26233

Garbet X 2001 Plasma Phys. Control. Fusion 43 A 251

Garbet X 2002 Private Communication

Garbet X et al 1992 Phys. Fluids B 4136

Garbet X et al 2001 Phys. Plasmas 82793

Gates D A et al 1997 Nucl. Fusion 371593

Geraud A et al 1994 Proc. 21 st Eur. Conf. on Controlled Fusion and Plasma Physics (Montpellier, 1994) vol. 18B, (part I), (Geneva: European Physical Society) p. 298

Giannella R et al 1994 Nucl. Fusion 341185

Gibson A et al 1998 Phys. Plasmas 51839

Giroud C et al 2001 28th Conf. on Controlled Fusion and Plasma Physics (Madeira, 2001), P2.018 (EPS)

Glasser A H, Greene J M and Johnson J L 1976 Phys. Fluids 19567 
Goldston R J 1984 Plasma Phys. Control. Fusion 2687

Goldston R J et al 1987 Proc. 11th IAEA Conf. on Plasma Phys. and Nucl. Fusion Research (Kyoto, 1986), vol. 1, (IAEA) p 75

Gormezano C 1993 Plasma Phys. Control. Fusion 35 A 239

Gormezano C 1999 Plasma Phys. Control. Fusion 41 B 367

Gormezano C et al 1998 Phys. Rev. Lett. 805544

Greenfield C M et al 1997 Phys. Plasmas 41596

Greenfield C M et al 1999 Nucl. Fusion 391723

Greenfield C M et al 2000a Phys. Plasmas 71959

Greenfield C M et al 2000b 27th Conf. on Controlled Fusion and Plasma Physics (Budapest, 2000) (EPS)

Greenfield C M et al 2001 Phys. Rev. Lett. 864544

Greenwald M et al 1984 Phys. Rev. Lett. $\mathbf{5 3} 352$

Greenwald M et al 1988 Nucl. Fusion 282199

Grieger G et al 1992 Phys. Fluids B 42081

Gruber O et al 1995 Phys. Rev. Lett. 744217

Gruber O et al 1999a Nucl. Fusion 391321

Gruber O et al 1999b Phys. Rev. Lett. 831787

Günter S et al 1998 Nucl. Fusion 381431

Günter S et al 1999 Nucl. Fusion 391535

Günter S et al 2000a Nucl. Fusion 401541

Günter S et al 2001a Nucl. Fusion 411283

Günter S et al 2001b in Nucl. Fusion submitted

Günter S et al 2000b Phys. Rev. Lett. 843097

H-mode Database Working Group 1994 Nucl. Fusion 34131

Hahm T S and Burrell K H 1995 Phys. Plasmas 21648

Hahm T S, Burrell K H, Greenfield C M and Synkowski E J 1998 Plasma Phys. Control. Fusion 40657

Hawkes N C et al 1992 Rev. Sci. Instrum. 635164

Hawkes N C et al 1999 Rev. Sci. Instrum. 70894

Hawkes N C et al 2001 Phys. Rev. Lett. 87115001

Hazeltine R D 1989 Phys. Fluids B 12031

Hellermann M G et al 1990 Rev. Sci. Instrum. 613479

Hellermann M G et al 1995 Plasma Phys. Control. Fusion 3771

Hellermann M G and Summers H P 1992 Rev. Sci. Instrum. 635132

Hender T C et al 1992 Nucl. Fusion 322091

Hender T C et al 2002 Plasma Phys. Control. Fusion 441143

Hill K W et al 1999 Phys. Plasmas 6877

Hinton F L and Hazeltine R D 1976 Rev. Mod. Phys. 48239

Hirshman S P 1988 Phys. Fluids 313150

Hirshman S P and Sigmar D J 1981 Nucl. Fusion 211079

Hoang G T et al 1998 Nucl. Fusion 38117

Hoang G T et al 2000 Phys. Rev. Lett. 844593

Hoang G T et al 2001 Phys. Rev. Lett. 87120001

Hobirk J et al 2001 Phys. Rev. Lett. 87085002

Hogeweij G M D et al 1998 Nucl. Fusion 381881

Hogeweij G M D et al 2002 Plasma Phys. Control. Fusion 441155

Horton W, Hong B G and Tang W M 1988 Phys. Fluids 312971

Houlberg W A, Shiang K C, Hirshman S P and Zarnstorff M C 1997 Phys. Plasmas 43230

Hubbard A et al 2001 Phys. Plasmas 82033

Hubbard A E 2000 Plasma Phys. Control. Fusion 42 A 15

Hugon M et al 1992 Nucl. Fusion 3233

Huysmans G T A et al 1999 Nucl. Fusion 391489

Ida K et al 1987 Phys. Rev. Lett. 58116

Ide S et al 2000 Phys. Plasmas 71927

Ikeda Y et al 2001 Fusion Engineering and Design 53351

In Y et al 2000 Nucl. Fusion 401463

Ishida S et al 1997 Phys. Rev. Lett. 793917

Ishii Y et al 1998 Plasma Phys. Control. Fusion 401607

ITER Confinement Database and Modelling Working Group 1997 Plasma Phys. Control. Fusion 39 B 115 
ITER Physics Expert Groups 1999 Nucl. Fusion 392175

Itoh S et al 1999 Nucl. Fusion 391257

Iwase M et al 1999 Plasma Phys. Control. Fusion 411189

Jachmich S, Van Oost G, Weynants R R and Boedo J A 1998 Plasma Phys. Control. Fusion 401105

Jackson G L et al 1991 Phys. Rev. Lett. 673098

Jackson G L et al 1999 Jour. Nucl. Mater. 266-269 380

Jaenicke R et al 1993 Nucl. Fusion 33687

Jenko F, Dorland W and Hammett G W 2001 Phys. Plasmas 84096

Jenko F et al 2000 Phys. Plasmas 71904

JET Team (prepared by C Gormezano) 1999 Nucl. Fusion 391875

JET Team (presented by C Gormezano) 1997 Proc. 16th IAEA Fusion Energy Conf. (Montreal, 1997) vol. I, p 487

(IAEA)

JET Team (presented by F X Söldner) 1997 Plasma Phys. Control. Fusion 39 B 353

JET Team (presented by F X Söldner) 1999 Nucl. Fusion 391883

JET Team (presented by G L Schmidt) 1989 Proc. 12th Int. Conf. on Plasma Phys. and Controlled Nuclear Fusion

Research (Nice, 1988) vol. 1, (Vienna: IAEA) p 215.

Joffrin E et al 2001 Plasma Phys. Control. Fusion submitted

Joffrin E et al 2002a Nucl. Fusion $\mathbf{4 2} 235$

Joffrin E et al 2002b Plasma Phys. Control. Fusion 441203

Kadomtsev B B and Pogutse O P 1967 Sov. Phys. JETP 241172

Kamada Y et al 1991 Nucl. Fusion 3123

Kamada Y et al 1994 Nucl. Fusion 341605

Kamada Y et al 1999a Plasma Phys. Control. Fusion 41 B 77

Kamada Y et al 1999b Nucl. Fusion 391845

Kamada Y et al 2000 Plasma Phys. Control. Fusion 42 A 247

Kardaun O J W F 1999 Plasma Phys. Control. Fusion 41429

Keilhacker M et al 1999 Nucl. Fusion 39202

Kessel C, Manickam J, Rewoldt G and Tang W M 1994 Phys. Rev. Lett. 721212

Kikuchi M and Azumi M 1995 Plasma Phys. Control. Fusion 371215

Kim J et al 1994 Phys. Rev. Lett. 722199

Kim Y B, Diamond P H and Groebner R J 1991 Phys. Fluids B 32050

Kinsey J E et al 2001 Phys. Rev. Lett. 86814

Koide Y, Burrell K H, Rice B W and Fujita T 1998a Plasma Phys. Control. Fusion 4097

Koide Y et al 1994 Phys. Rev. Lett. 723662

Koide Y et al 1996 Plasma Phys. Control. Fusion 381011

Koide Y et al 1997 Phys. Plasmas 41623

Koide Y et al 1998b Plasma Phys. Control. Fusion 40641

Koslowski H R et al 1997 Plasma Phys. Control. Fusion 39 B 325

Kotschenreuther M, Dorland W, Beer M A and Hammett G W 1995 Phys. Plasmas 2381

Kusama Y et al 1999 Plasma Phys. Control. Fusion 41625

La Haye R J et al 1995 Nucl. Fusion 35988

Lackner K, Günter, S, Jenko F, Peeters A and Wolf R C 2000 Plasma Phys. Control. Fusion 42 B 37

LaHaye R J et al 1994 Phys. Plasmas 1373

LaHaye R J and Sauter O 1998 Nucl. Fusion 38987

Lao L L et al 1993 Phys. Rev. Lett. 703435

Lao L L et al 1996a Phys. Plasmas 31951

Lao L L et al 1996b Plasma Phys. Control. Fusion 381439

Lao L L et al 1998 Phys. Plasmas 51050

Lazarus E A et al 1985 Nucl. Fusion 25135

Lazarus E A et al 1991 Phys. Fluids B 32220

Lazarus E A et al 1996 Phys. Rev. Lett. 772714

LeBlanc B et al 1995 Phys. Plasmas 2741

LeBlanc B et al 1999 Phys. Rev. Lett. 82331

Leuterer F et al 1985 Phys. Rev. Lett. 5575

Levinton F M 1999 Rev. Sci. Instrum. 70810

Levinton F M et al 1989 Phys. Rev. Lett. 632060

Levinton F M et al 1995 Phys. Rev. Lett. 754417

Levinton F M et al 1998 Phys. Rev. Lett. 804887 
Lin Z, Tang W M and Lee W W 1995 Phys. Plasmas 22975

Lin Z, Tang W M and Lee W W 1997a Phys. Plasmas 41707

Lin Z, Tang W M and Lee W W 1997b Phys. Rev. Lett. 78456

Litaudon X et al 1996 Plasma Phys. Control. Fusion 381603

Litaudon X et al 1999 Plasma Phys. Control. Fusion 41 A 733

Litaudon X et al 2002 Plasma Phys. Control. Fusion 441057

Lomas P et al 2001 28th Conf. on Controlled Fusion and Plasma Physics (Madeira, 2001), P2.023 (EPS)

Lopes Cardozo N J et al 1997 Plasma Phys. Control. Fusion 39 B 303

Lopez Cardozo N J 1995 Plasma Phys. Control. Fusion 37799

Maget P, Garbet X, Géraud, A and Joffrin E 1999 Nucl. Fusion 39949

Manickam J et al 1997 Proc. 16th IAEA Conf. on Plasma Phys. and Nucl. Fusion Research (Montreal, 1996) vol. 1 (IAEA) p 453

Manickam J et al 1999 Nucl. Fusion 391819

Manickam J, Pomphrey N and Todd A M M 1987 Nucl. Fusion 271461

Mantsinen M J et al 2002 Phys. Rev. Lett. 88105002

Marmar E S et al 1982 Nucl. Fusion 221567

Mattioli M et al 1998 Nucl. Fusion 381629

Mazon D et al 2002 Plasma Phys. Control. Fusion 441087

Mazzucato E et al 1996 Phys. Rev. Lett. 773145

McKee G et al 1999 Rev. Sci. Instrum. 70913

McKee G et al 2000a Phys. Plasmas 71870

McKee G et al 2000b Phys. Rev. Lett. 841922

Meister H et al 2001 Nucl. Fusion 411633

Messiaen A M et al 1996 Phys. Rev. Lett. 772487

Moreau D and Voitsekhovitch I 1999 Nucl. Fusion 39685

Nazikian R et al 1999 17th IAEA Conf. on Fusion Energy (Yokohama, 1998), Post deadline (IAEA)

Nazikian R and Mazzucato E 1995 Rev. Sci. Instrum. 66392

Neudatchin S V et al 1999 Plasma Phys. Control. Fusion 41 L39

Newman D E et al 1998 Phys. Plasmas 5938

Oikawa T et al 2000 Nucl. Fusion 40435

Okumura Y et al 2000 Rev. Sci. Instrum. 711219

Ongena J et al 1993 Nucl. Fusion 33283

Ozeki T, Azumi M, Ishida S and Fujita T 1998 Plasma Phys. Control. Fusion 40871

Ozeki T et al 1997 Plasma Phys. Control. Fusion 39 A 371

Paccagnella R, Romanelli F and Briguglio S 1990 Nucl. Fusion 30545

Parail V V et al 1999 Nucl. Fusion 39429

Paul S F and Fonck R J 1990 Rev. Sci. Instrum. 613496

Peacock N J et al 1969 Nature 224488

Peeters A G 2000 Plasma Phys. Control. Fusion 42 B 231

Peeters A G et al 2001a 18th IAEA Conf. on Fusion Energy (Sorrento, 2000), IAEA-CN-EXP5/06 (IAEA)

Peeters A G et al 2001b Nucl. Fusion submitted

Pericoli-Ridolfini V et al 1999 Phys. Rev. Lett. 8293

Phillips M W et al 1996 Phys. Plasmas 31673

Pietrzyk Z A et al 2001 Phys. Rev. Lett. 861530

Porcelli F, Rossi E, Cima G and Wootton A 1999 Phys. Rev. Lett. 821458

Prater R et al 2001 18th IAEA Conf. on Fusion Energy (Sorrento, 2000), IAEA-CN-77/EX8/1 (IAEA)

Rapp J et al 1997 Plasma Phys. Control. Fusion 391615

Redd A J, Kritz A H, Bateman G, Rewoldt G and Tang W M 1999 Phys. Plasmas 61162

Rettig C L et al 1998 Phys. Plasmas 51727

Rice B W et al 1995 Rev. Sci. Instrum. 66373

Rice B W et al 1996a Phys. Plasmas 31983

Rice B W et al 1996b Nucl. Fusion 361271

Rice B W et al 1996c Plasma Phys. Control. Fusion 38869

Rice B W et al 1997 Phys. Rev. Lett. 792694

Rice B W et al 1999a Nucl. Fusion 391855

Rice B W et al 1999b Rev. Sci. Instrum. 70815

Rice J E et al 2001 Nucl. Fusion 41277

Romanelli F 1989 Plasma Phys. Control. Fusion 311535 
Ruskov E et al 1999 Phys. Rev. Lett. 82924

Ryter F et al 2001a Phys. Rev. Lett. 862325

Ryter F et al 2001b Plasma Phys. Control. Fusion 43 A 323

Ryter F et al 2001c Nucl. Fusion 41537

Ryter F et al 2001d Phys. Rev. Lett. 865498

Ryter F et al 2002 Plasma Phys. Control. Fusion 44 A 415

Saibene G, Horton L D, Sartori R et al 1999 Nucl. Fusion 391133

Sakamoto Y et al 2001 18th IAEA Conf. on Fusion Energy (Sorrento, 2000), IAEA-CN-EX5/04 (IAEA)

Saoutic B et al 1997 Proc. 16th IAEA Conf. on Plasma Phys. and Nucl. Fusion Research (Montreal, 1996), vol. 1, (IAEA) p 141

Sarazin Y et al 2000 Proc. 27th Eur. Conf. on Controlled Fusion and Plasma Physics (Budapest, 2000) (Geneva: European Physical Society) p 253

Sattler S and Hartfuss H J 1993 Plasma Phys. Control. Fusion 351285

Sauter O and Angioni C 1999 Phys. Plasmas 62834

Sauter O et al 2000 Phys. Rev. Lett. 843322

Sauter O et al 2001 Phys. Plasmas 82199

Schüller, F C et al 2000 27th Conf. on Controlled Fusion and Plasma Physics (Budapest, 2000) (EPS)

Shaing K C, Hazeltine R D and Zarnstorff M C 1997 Phys. Plasmas 4771

Shirai H et al 1998 Phys. Plasmas 51712

Shirai H et al 1999 Nucl. Fusion 391713

Shirai H et al 2000 Plasma Phys. Control. Fusion 42 A 109

Sips A C C et al 1998 Plasma Phys. Control. Fusion 401171

Söldner, F X et al 1999 Nucl. Fusion 39407

Soltwisch H 1992 Plasma Phys. Control. Fusion 341669

Staebler G M 2000 Phys. Rev. Lett. 843610

Staebler G M et al 1999 Phys. Rev. Lett 821692

Staebler G M et al 2001 Nucl. Fusion 41891

Staebler G M, Waltz R E et al 1998 Proc. 25th Eur. Conf. on Controlled Fusion and Plasma Physics (Praha, 1998) vol. 22C, (Geneva: European Physical Society) p 2006

Staebler G M, Waltz R E and Wiley J C 1997a Nucl. Fusion 37287

Staebler G M, Waltz R E and Wiley J C 1997b Nucl. Fusion 37287

Stallard B W et al 1999 Phys. Plasmas 61978

Stober J et al 2000 Plasma Phys. Control. Fusion 42 A 211

Stober J et al 2001a Nucl. Fusion 411123

Stober J et al 2001b Nucl. Fusion 411535

Stober J et al 2002 Plasma Phys. Control. Fusion 44 A 159

Strachan J D et al 1987 Phys. Rev. Lett. 581004

Strachan J D et al 1994 Plasma Phys. Control. Fusion 36 B 3

Strait E J et al 1995 Phys. Rev. Lett. 754421.

Strait E J et al 1997 Phys. Plasmas 41783

Stratton B C et al 1999 Nucl. Fusion 391309

Suttrop W et al 1997 Plasma Phys. Control. Fusion 392051

Suttrop W et al 2001 28th Conf. on Controlled Fusion and Plasma Physics (Madeira, 2001), P3.016 (EPS)

Synakowski E J 1998 Plasma Phys. Control. Fusion 40581

Synakowski E J et al 1997a Phys. Rev. Lett. 782972

Synakowski E J et al 1997b Phys. Plasmas 41736

Synakowski E J et al 1999 Nucl. Fusion 391733

Takase Y et al 1996 Plasma Phys. Control. Fusion 382215

Takeji S et al 1997 Phys. Plasmas 44283

Takenaga H et al 1998 Plasma Phys. Control. Fusion 40183

Tala T J J, Heikkinen J A, Parail V V et al 2001 Plasma Phys. Control. Fusion 43507

Tala T J J, Parail V V et al 2002 Plasma Phys. Control. Fusion 441181

Taylor R J, Brown M L, Fried B D, Grote H, Liberati J R, Morales G J, Pribyl P, Darrow D and Ono M 1989 Phys. Rev. Lett. $\mathbf{6 3} 2365$

Taylor T S 1997 Plasma Phys. Control. Fusion 39 B 47

Taylor T S et al 1991 Proc. 13th IAEA Conf. on Plasma Phys. and Nucl. Fusion Research (Washington DC, 1990) vol. 1, (IAEA) p 177 
The T-10 Team (presented by D A Kislov) 2001 18th IAEA Conf. on Fusion Energy (Sorrento, 2000), IAEA-CN$77 / O V 5 / 2$ (IAEA)

Tobita K et al 1997 Nucl. Fusion 371583

Tokar M Z et al 1999 Plasma Phys. Control. Fusion 41 B 317

Tresset G et al 2001 28th Conf. on Controlled Fusion and Plasma Physics (Madeira, 2001) (EPS)

Tresset G et al 2002 Nucl. Fusion 42520

Troyon F et al 1984 Plasma Phys. Control. Fusion 26209

Tubbing B J D et al 1991 Nucl. Fusion 31839

van Blokland A A E, Azumi M, Kikuchi M, Tani K and Tobita K 1994 Plasma Phys. Control. Fusion 36925

Voitsekhovitch I et al 1997 Nucl. Fusion 371715

Voitsekhovitch I and Moreau D 2001 Nucl. Fusion 41845

Vollmer O et al 2000 Rev. Sci. Instrum. 71939

Wagner F et al 1982 Phys. Rev. Lett. 491408

Wagner F et al 1995 Trans. Fusion Technology 29407

Wakatani M 1998 Plasma Phys. Control. Fusion 40597

Waltz R E, Dewar R L and Garbet X 1998 Phys. Plasmas 51784

Waltz R E et al 1995 Phys. Plasmas 2408

Waltz R E, Kerbel G D and Milovich J 1994 Phys. Plasmas 12229

Ward D J and Bondeson A 1995 Phys. Plasmas 21570

Weisen H et al 1991 Nucl. Fusion 312247

Wesson J 1997 Tokamaks 2nd edn (Oxford: Clarendon)

Weynants R R et al 1999 Nucl. Fusion 391637

Weynants R R, Jachmich S and Van Oost G 1998 Plasma Phys. Control. Fusion 40635

Wilson H R and Miller R L 1999 Phys. Plasmas 6873

Wobig H et al 1987 Proc. 11th IAEA Conf. on Plasma Phys. and Nucl. Fusion Research (Kyoto, 1986) vol. 2, (IAEA) p 369

Wolf R C et al 1997 Proc. 24th Eur. Conf. on Controlled Fusion and Plasma Physics (Berchtesgaden, 1997) (Geneva: European Physical Society) p 1509

Wolf R C et al 1999a Proc. 17th IAEA Fusion Energy Conf. (Yokohama, 1999) (IAEA) p 733

Wolf R C et al 1999b Plasma Phys. Control. Fusion 41 B 93

Wolf R C et al 2000 Phys. Plasmas 71839

Wolf R C et al 2001a Nucl. Fusion 411259

Wolf R C et al 2001b 28th Conf. on Controlled Fusion and Plasma Physics (Madeira, 2001), P2.009 (EPS)

Wolfe S M, Greenwald M et al 1986 Nucl. Fusion 26329

Wroblewski D et al 1990 Rev. Sci. Instrum. 613552

Yoshizawa A, Itoh S, Itoh K and Yokoi N 2001 Plasma Phys. Control. Fusion 43 R1

Yu Q and Günter, S 1999 Nucl. Fusion 39487

Yushmanov P N et al 1990 Nucl. Fusion 301999.

Zarnstorff M C et al 1993 14th IAEA Conf on Plasma Phys. and Control. Nucl. Fusion Research (Würzburg, 1992), p 111 (IAEA)

Zarnstorff M C et al 1997 Phys. Plasmas 41097

Zastrow K-D et al 1999 26th Conf. on Controlled Fusion and Plasma Physics (Maastricht, 1999) (EPS)

Zhu P, Horton W and Sugama H 1999 Phys. Plasmas 62503

Zohm H 1996 Plasma Phys. Control. Fusion 105

Zohm H, Gates D A and Wilson H R 1997 Plasma Phys. Control. Fusion 39 B 237

Zweben S J, Darrow D S et al 1998 Nucl. Fusion 38739 